Oxidative C-H/N-H Annulation of Aromatic Amides with Dialkyl Malonates: Access to Isoindolinones and Dihydrobenzoindoles

Sourav Pradhan, Subhasish Roy, Sonbidya Banerjee, Pinaki Bhusan De and Tharmalingam Punniyamurthy* Department of Chemistry, Indian Institute of Technology Guwahati, Guwahati 781039, India *E-mail: tpunni@iitg.ac.in

\title{
Table of Contents
}

1 Figure S1: Biologically Active Compounds $\quad$ S2

2 Table S1 and Table S2: Optimization Studies $\quad$ S2-S4

3 Scheme S1. Preliminary Mechanistic Investigations $\quad$ S4-S5

4 Scheme S2: Synthetic Utilities

5 Crystal Data and Structure Refinement for 3c S6-S7

6 NMR Spectra $\quad$ S8-S48 
Isoindolinones

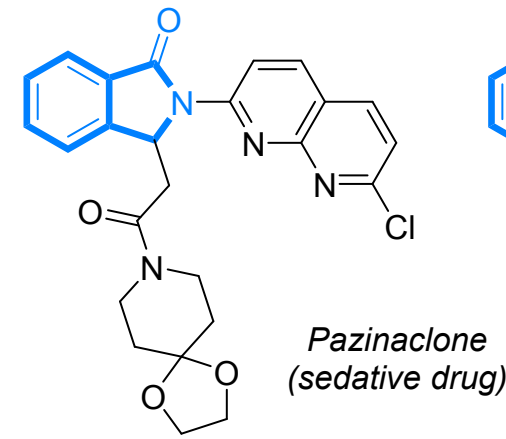<smiles>CCCCC=O</smiles><smiles>CC(C(=O)O)c1ccc(N2Cc3ccccc3C2=O)cc1</smiles>

Dihydrobenzoindoles<smiles>CC(=O)n1c(=C(C#N)C#N)c2cccc3cccc1c32</smiles>

Antitumor agent

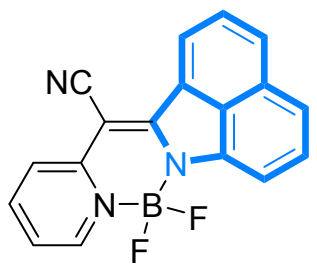

Fluorescent dyes

Figure S1. Biologically Active Isoindolinones and Dihydrobenzoindoles.

Table S1. Optimization of the Reaction Conditions with 1a and $\mathbf{2} \mathbf{a}^{a}$<smiles>Nc1cccc2ccccc12</smiles>

$1 \mathrm{a}$<smiles>CCOC(=O)CCCCOCC(=O)OCC</smiles>

$2 a$<smiles>CCCN1c2cccc3cccc(c23)C1(C(=O)OCC)C(=O)OCC</smiles>

$3 a$<smiles>[Y]C(=O)c1ccccn1</smiles>

(PA) $\mathrm{N}$

\begin{tabular}{lllll}
\hline Entry & {$[\mathrm{Cu}]$ Source } & Base & Solvent & Yield $(\%)^{b}$ \\
\hline 1 & $\mathrm{CuCl}_{2}$ & $\mathrm{Na}_{2} \mathrm{CO}_{3}$ & DMSO & n.d. \\
2 & $\mathrm{CuBr}_{2}$ & $\mathrm{Na}_{2} \mathrm{CO}_{3}$ & DMSO & n.d. \\
3 & $\mathrm{Cu}(\mathrm{OAc})_{2}$ & $\mathrm{Na}_{2} \mathrm{CO}_{3}$ & DMSO & 32 \\
4 & $\mathrm{CuOAc}$ & $\mathrm{Na}_{2} \mathrm{CO}_{3}$ & DMSO & 21 \\
5 & $\mathrm{Cu}(\mathrm{OAc})_{2}$ & $\mathrm{Cs}_{2} \mathrm{CO}_{3}$ & DMSO & trace \\
6 & $\mathrm{Cu}(\mathrm{OAc})_{2}$ & $\mathrm{~K}_{3} \mathrm{PO}_{4}$ & DMSO & trace \\
7 & $\mathrm{Cu}(\mathrm{OAc})_{2}$ & $\mathrm{CsOPiv}^{2}$ & DMSO & 56 \\
$\mathbf{8}$ & $\mathbf{C u}(\mathbf{O A c})_{2}$ & $\mathbf{N a O P i v} \bullet \mathbf{H}_{2} \mathbf{O}$ & DMSO & $\mathbf{6 7}$
\end{tabular}




$\begin{array}{lllll}9^{c} & \mathrm{Cu}(\mathrm{OAc})_{2} & \mathrm{NaOPiv} \cdot \mathrm{H}_{2} \mathrm{O} & \mathrm{DMSO} & 14 \\ 10^{d} & \mathrm{Cu}(\mathrm{OAc})_{2} & \mathrm{NaOPiv} \cdot \mathrm{H}_{2} \mathrm{O} & \mathrm{DMSO} & 16 \\ 11 & \mathrm{Cu}(\mathrm{OAc})_{2} & \mathrm{Na}_{2} \mathrm{CO}_{3} & \mathrm{DMF} & \text { trace } \\ 12 & \mathrm{Cu}(\mathrm{OAc})_{2} & \mathrm{Na}_{2} \mathrm{CO}_{3} & \mathrm{PhCl} & \text { trace } \\ 13^{e} & \mathrm{Cu}(\mathrm{OAc})_{2} & \mathrm{NaOPiv} \cdot \mathrm{H}_{2} \mathrm{O} & \mathrm{DMSO} & 23 \\ 14^{f} & \mathrm{Cu}(\mathrm{OAc})_{2} & \mathrm{NaOPiv} \cdot \mathrm{H}_{2} \mathrm{O} & \text { DMSO } & 51 \\ 15 & \mathrm{Cu}(\mathrm{OAc})_{2} & - & & \text { trace } \\ 16 & - & \mathrm{NaOPiv} \cdot \mathrm{H}_{2} \mathrm{O} & \text { DMSO } & \text { n.d. }\end{array}$

${ }^{a}$ Reaction conditions: 1a $(0.1 \mathrm{mmol}), \mathbf{2 a}(0.2 \mathrm{mmol}),[\mathrm{Cu}](0.1 \mathrm{mmol})$, base $(0.2$ mmol), solvent $(2 \mathrm{~mL}), 120{ }^{\circ} \mathrm{C}$, air, 4 h. ${ }^{b}$ Isolated yield. ${ }^{c} 20 \mathrm{~mol} \% \mathrm{Cu}(\mathrm{OAc})_{2}$ and 1 equiv $\mathrm{Ag}_{2} \mathrm{CO}_{3}$ used. ${ }^{d} 20 \mathrm{~mol} \% \mathrm{Cu}(\mathrm{OAc})_{2}$ and 1 equiv NMO. ${ }^{e}$ Under $\mathrm{N}_{2}$ atm. $f$ Under $\mathrm{O}_{2}$ atm. n.d. $=$ not detected. $\mathrm{NMO}=N$-Methylmorpholine $N$-oxide.

Table S2. Optimization of the Reaction Conditions with $\mathbf{4 a}$ and $\mathbf{2} \mathbf{a}^{a}$<smiles>O=C(Nc1cccc2cccnc12)c1ccccc1</smiles>

$4 a$<smiles>CCOC(=O)CC(=O)OCC</smiles>

2a

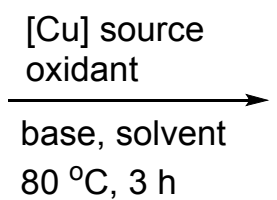

$80^{\circ} \mathrm{C}, 3 \mathrm{~h}$<smiles>CCOC(=O)C1(OCC)c2ccccc2C(=O)N1O</smiles>

5a

\begin{tabular}{|l|l|l|l|l|l|}
\hline Entry & {$[\mathrm{Cu}]$ Source } & Base & Oxidant & Solvent & Yield (\%) $)^{b}$ \\
\hline 1 & $\mathrm{CuCl}_{2}$ & $\mathrm{Na}_{2} \mathrm{CO}_{3}$ & $\mathrm{Ag}_{2} \mathrm{CO}_{3}$ & DMSO & n.d. \\
\hline 2 & $\mathrm{CuBr}_{2}$ & $\mathrm{Na}_{2} \mathrm{CO}_{3}$ & $\mathrm{Ag}_{2} \mathrm{CO}_{3}$ & DMSO & n.d. \\
\hline $\mathbf{3}$ & $\mathbf{C u}(\mathbf{O A c})_{2}$ & $\mathrm{Na}_{2} \mathrm{CO}_{3}$ & $\mathbf{A g}_{2} \mathbf{C O}_{3}$ & DMSO & $\mathbf{6 5 \%}$ \\
\hline $4^{c}$ & $\mathrm{Cu}(\mathrm{OAc})_{2}$ & $\mathrm{Na}_{2} \mathrm{CO}_{3}$ & $\mathrm{Ag}_{2} \mathrm{CO}_{3}$ & DMSO & $63 \%$ \\
\hline $5^{d}$ & $\mathrm{Cu}(\mathrm{OAc})_{2}$ & $\mathrm{Na}_{2} \mathrm{CO}_{3}$ & $\mathrm{Ag}_{2} \mathrm{CO}_{3}$ & DMSO & $63 \%$ \\
\hline 6 & $\mathrm{CuOAc}$ & $\mathrm{Na}_{2} \mathrm{CO}_{3}$ & $\mathrm{Ag}_{2} \mathrm{CO}_{3}$ & DMSO & 16 \\
\hline
\end{tabular}




\begin{tabular}{|l|l|l|l|l|l|}
\hline 7 & $\mathrm{Cu}(\mathrm{OAc})_{2}$ & $\mathrm{~K}_{2} \mathrm{CO}_{3}$ & $\mathrm{Ag}_{2} \mathrm{CO}_{3}$ & DMSO & 31 \\
\hline 8 & $\mathrm{Cu}(\mathrm{OAc})_{2}$ & $\mathrm{Cs}_{2} \mathrm{CO}_{3}$ & $\mathrm{Ag}_{2} \mathrm{CO}_{3}$ & DMSO & n.d. \\
\hline 9 & $\mathrm{Cu}(\mathrm{OAc})_{2}$ & $\mathrm{~K}_{3} \mathrm{PO}_{4}$ & $\mathrm{Ag}_{2} \mathrm{CO}_{3}$ & DMSO & trace \\
\hline 10 & $\mathrm{Cu}(\mathrm{OAc})_{2}$ & $\mathrm{Na}_{2} \mathrm{CO}_{3}$ & $\mathrm{AgOAc}$ & DMSO & 15 \\
\hline 11 & $\mathrm{Cu}(\mathrm{OAc})_{2}$ & $\mathrm{Na}_{2} \mathrm{CO}_{3}$ & $\mathrm{NMO}$ & DMSO & 18 \\
\hline 12 & $\mathrm{Cu}(\mathrm{OAc})_{2}$ & $\mathrm{Na}_{2} \mathrm{CO}_{3}$ & $\mathrm{NHPI}$ & DMSO & n.d. \\
\hline 14 & $\mathrm{Cu}(\mathrm{OAc})_{2}$ & $\mathrm{Na}_{2} \mathrm{CO}_{3}$ & $\mathrm{O}_{2}$ & DMSO & 12 \\
\hline 15 & $\mathrm{Cu}(\mathrm{OAc})_{2}$ & $\mathrm{Na}_{2} \mathrm{CO}_{3}$ & $\mathrm{Ag}_{2} \mathrm{CO}_{3}$ & DMF & \multicolumn{2}{l|}{ trace } \\
\hline 16 & $\mathrm{Cu}(\mathrm{OAc})_{2}$ & $\mathrm{Na}_{2} \mathrm{CO}_{3}$ & $\mathrm{Ag}_{2} \mathrm{CO}_{3}$ & NMP & \multicolumn{2}{l|}{ n.d. } \\
\hline 17 & - & $\mathrm{Ag}_{2} \mathrm{CO}_{3}$ & m-xylene & 10 \\
\hline
\end{tabular}

${ }^{a}$ Reaction conditions: 1a $(0.1 \mathrm{mmol}), \mathbf{2 a}(0.2 \mathrm{mmol}),[\mathrm{Cu}](0.02 \mathrm{mmol})$, base $(0.2$ mmol), oxidant $(0.1 \mathrm{mmol})$, solvent $(2 \mathrm{~mL}), 80{ }^{\circ} \mathrm{C}$, air, 3 h. ${ }^{b}$ Isolated yield. ${ }^{c} 30$ mol $\% \mathrm{Cu}(\mathrm{OAc})_{2} \cdot{ }^{d} 40 \mathrm{~mol} \% \mathrm{Cu}(\mathrm{OAc})_{2}$. n.d. $=$ not detected.

\section{Scheme S1. Preliminary Mechanistic Investigations}

a. Control experiments<smiles>CCOC(=O)C(C(=O)OCC)c1ccccc1C(=O)NO</smiles>

standard conditions<smiles>CCOC(=O)C1(C(=O)OCC)c2ccccc2C(=O)N1O</smiles>

5a, $73 \%$<smiles>Cc1cc(C)c(C(=O)NO)c([N+](=O)[O-])c1</smiles><smiles>CCOC(=O)C(C(=O)OCC)N(O)C(=O)c1c(C)cc(C)cc1C</smiles> 
b. Competitive experiment: EDG/EWG

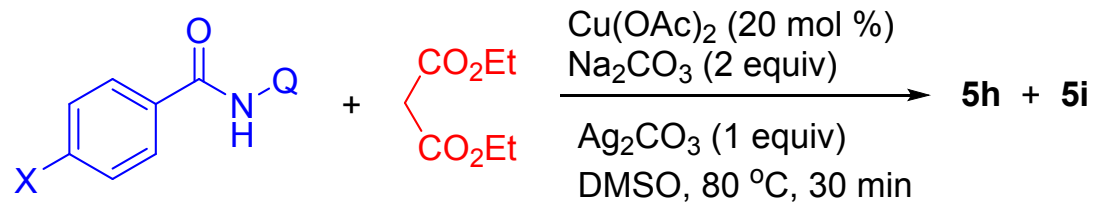

4h, $X=O M e$

$4 i, X=\mathrm{NO}_{2}$

$2 \mathrm{a}$

$\mathbf{5 h}: 5 \mathbf{i}=1: 1.2$

c. Kinetic isotope experiments<smiles>CCCNc1cccc2cccc(O)c12</smiles>

$1 a / 1 a-d$<smiles>CCOCCC(=O)OCC</smiles>

$\mathrm{Cu}(\mathrm{OAc})_{2}$ (1 equiv) $\mathrm{NaOPiv} \bullet \mathrm{H}_{2} \mathrm{O}$ (2 equiv)

DMSO, $120^{\circ} \mathrm{C}, 35 \mathrm{~min}$

$$
k_{\mathrm{H}} / k_{\mathrm{D}}=1.14
$$<smiles>Oc1cccc2c1=C[C+]=CC=2</smiles>

recovered 1a/1a-d

\section{Scheme S2. Synthetic Utilities}

Scale-up synthesis<smiles>CCCNc1cccc2ccccc12</smiles>

1a $(2 \mathrm{mmol})$

$$
\begin{aligned}
& +\sum_{\mathrm{CO}_{2} \mathrm{Et}}^{\mathrm{CO}_{2} \mathrm{Et}} \stackrel{\mathrm{Cu}(\mathrm{OAc})_{2} \text { (1 equiv) }}{\mathrm{NaOPiv} \bullet \mathrm{H}_{2} \mathrm{O}(2 \text { equiv })} \\
& \text { DMSO, } 120^{\circ} \mathrm{C}, 4 \mathrm{~h}
\end{aligned}
$$

$2 \mathrm{a}$<smiles>CCOC(=O)C1(C(=O)OCC)c2cccc3cccc(c23)N1c1ccccc1</smiles>

3a, $51 \%$

\section{Decarboxylation}<smiles>CCOC(=O)C1(OCC)c2ccccc2C(=O)N1O</smiles>

$5 a$

\section{$\frac{\text { CAN (3 equiv) }}{\mathrm{CH}_{3} \mathrm{CN} / \mathrm{H}_{2} \mathrm{O}(4: 1)}$} rt, $5 \mathrm{~h}$<smiles>CCOC(=O)C1c2ccccc2C(=O)N1O</smiles>

7, $62 \%$ 


\section{Crystal Data and Structure Refinement for 3c}

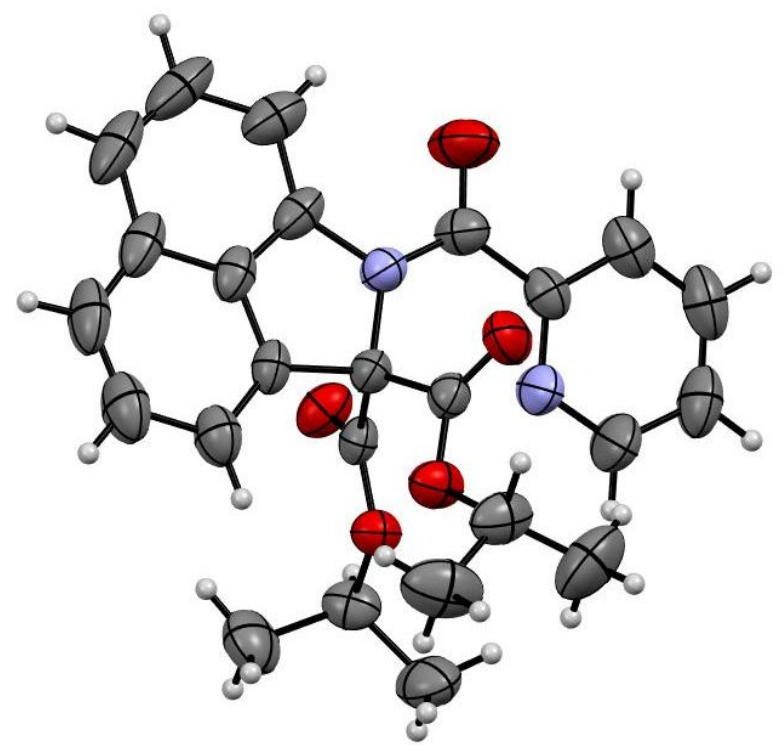

Figure S2. ORTEP diagram of diisopropyl 1-picolinoylbenzo[cd]indole-2,2(1H)dicarboxylate 3c with 50\% ellipsoid (CCDC 1981569).

Sample Preparation for Crystal Growth: The compound 3c were dissolved in acetonitrile and kept for slow evaporation at room temperature. Needle shaped crystals formed after 3 days. The single crystals were then subjected to X-ray diffraction analysis

\begin{tabular}{ll}
\hline Identification code & $\mathbf{3 c}$ \\
CCDC No. & $\mathbf{1 9 8 1 5 6 9}$ \\
Empirical formula & $\mathrm{C}_{25} \mathrm{H}_{24} \mathrm{~N}_{2} \mathrm{O}_{5}$ \\
Solvent for crystal growth & Acetonitrile \\
Formula weight & 432.46 \\
Crystal habit, colour & needle /colorless \\
Temperature, $T / \mathrm{K}$ & $296(2) \mathrm{K}$ \\
Wavelength, $\lambda / \AA$ & 0.71073 \\
Crystal system & triclinic \\
Space group & 'P -1'
\end{tabular}


Unit cell dimensions

Volume, $V / \AA^{3}$

Z

Calculated density, $\mathrm{Mg} \cdot \mathrm{m}^{-3}$

Absorption coefficient, $\mu / \mathrm{mm}^{-1}$

$F(000)$

$\theta$ range for data collection

Limiting indices

Reflection collected / unique

Completeness to $\theta$

Absorption correction

Refinement method

Data / restraints / parameters

Goodness-of-fit on $F^{2}$

Final $R$ indices $[I>2 \operatorname{sigma}(I)]$

$R$ indices (all data)

$$
\begin{aligned}
& a=9.2898(8) \AA \\
& b=11.6784(9) \AA \\
& c=12.1295(10) \AA \\
& \alpha=92.062(3) \\
& \beta=112.040(3) \\
& \gamma=110.021(3)
\end{aligned}
$$

1125.43(17)

2

1.276

0.090

456

2.38 to $20.95^{\circ}$

$-11 \leq h \leq 11,-13 \leq k \leq 13,-14 \leq l \leq 14$

$3959 / 2803$

$100 \%\left(\theta=24.995^{\circ}\right)$

none

'SHELXL-2014 (Sheldrick, 2014)'

$3960 / 0 / 293$

1.024

$R 1=0.0520, w R 2=0.1222$

$R 1=0.0805, w R 2=0.1443$ 
PS-1A-DG-1H<smiles>O=C(Nc1cccc2ccccc12)c1ccccn1</smiles>

$1 a$

${ }^{1} \mathrm{H}$ NMR $\left(400 \mathrm{MHz}, \mathrm{CDCl}_{3}\right)$

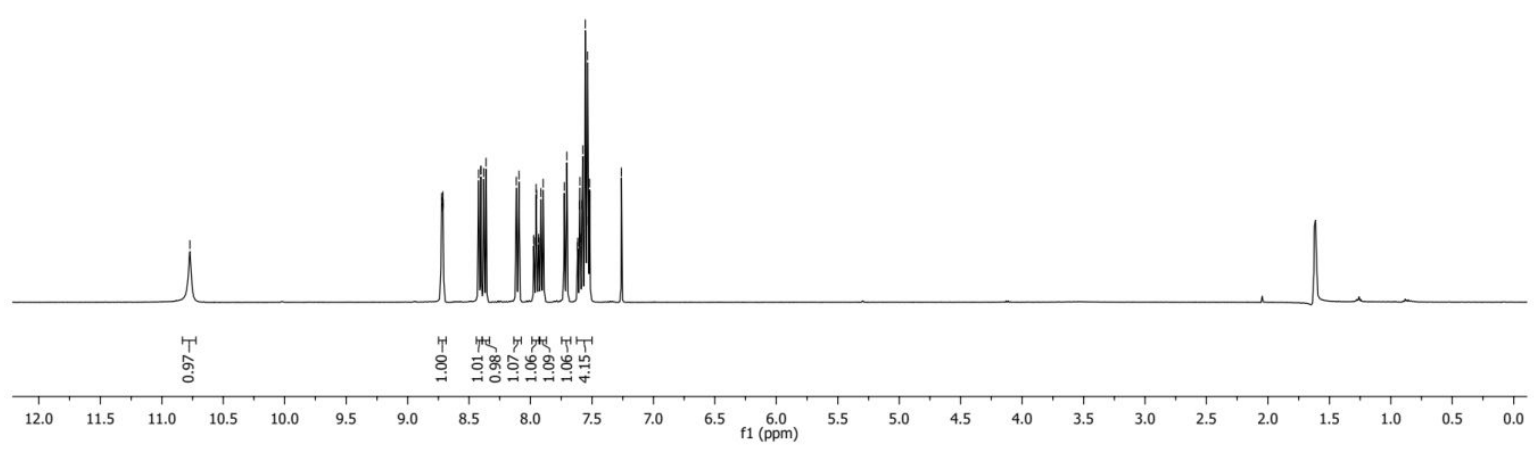

PS-1B-DG-1H

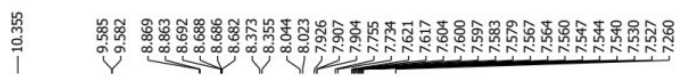

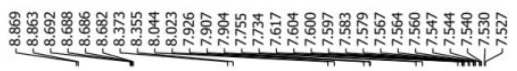

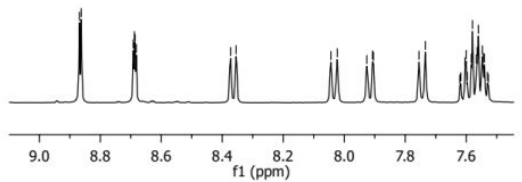<smiles>O=C(Nc1cccc2ccccc12)c1cnccn1</smiles>

$1 \mathrm{~b}$

${ }^{1} \mathrm{H}$ NMR $\left(400 \mathrm{MHz}, \mathrm{CDCl}_{3}\right)$

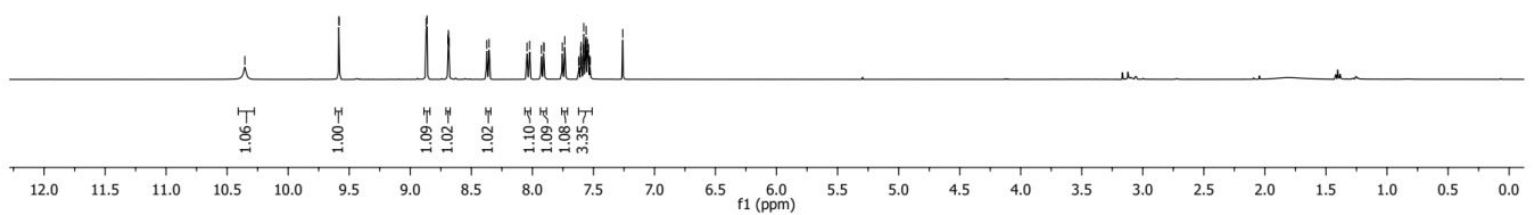




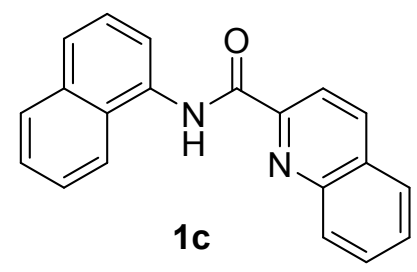

${ }^{1} \mathrm{H} \mathrm{NMR}\left(400 \mathrm{MHz}, \mathrm{CDCl}_{3}\right)$

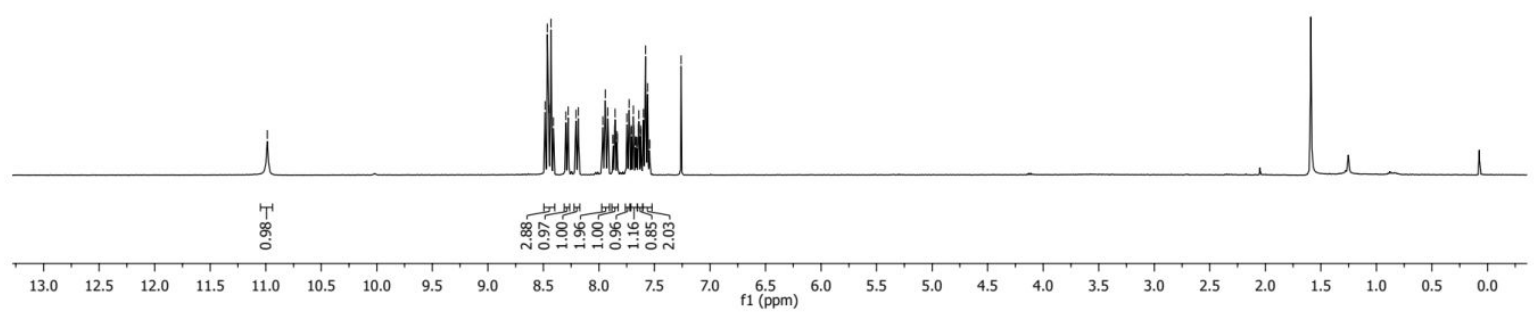

PS-1D-DG-1H

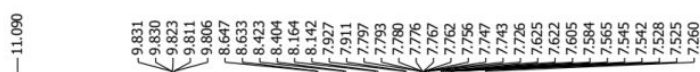<smiles>O=C(Nc1cccc2ccccc12)c1nccc2ccccc12</smiles>

1d

${ }^{1} \mathrm{H} \mathrm{NMR}\left(400 \mathrm{MHz}, \mathrm{CDCl}_{3}\right)$

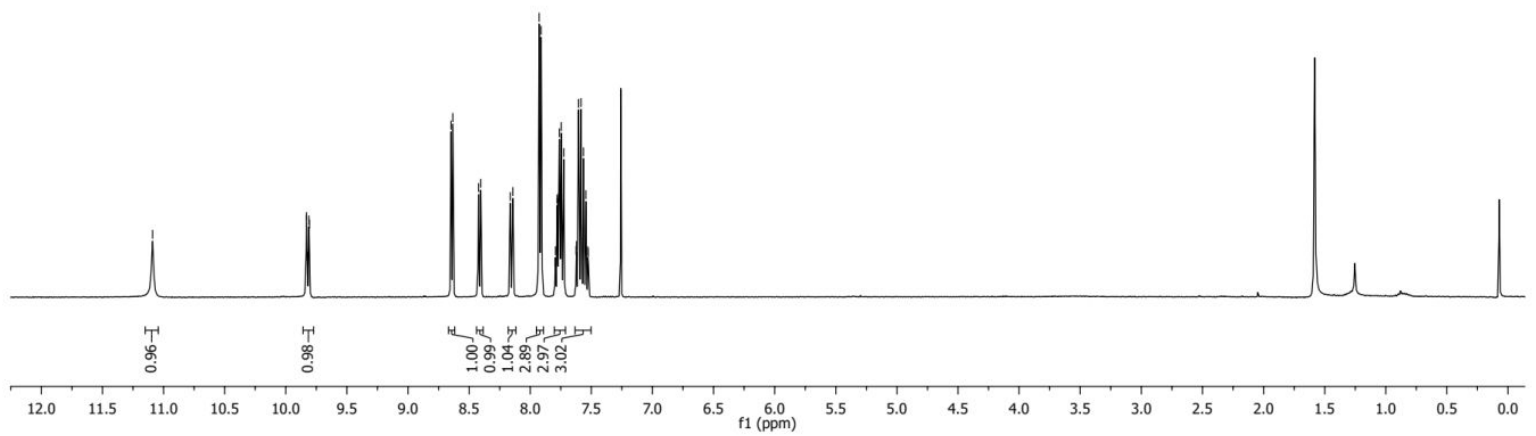



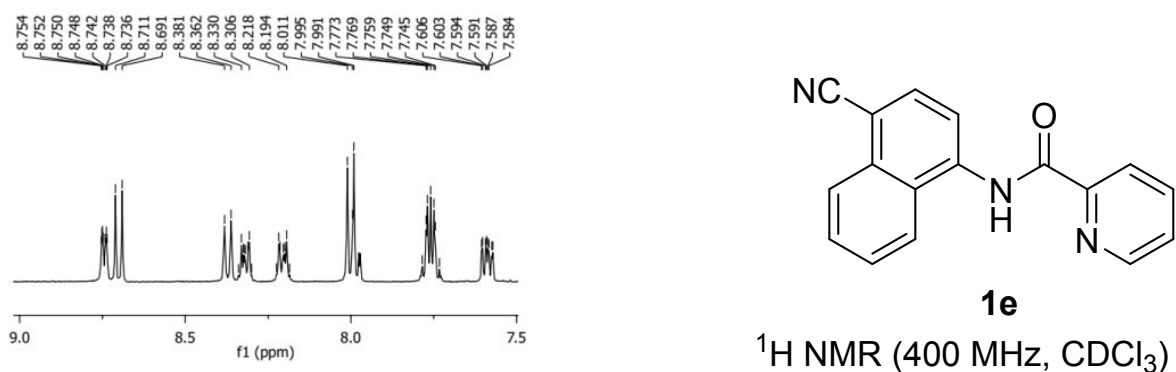

${ }^{1} \mathrm{H} \mathrm{NMR}\left(400 \mathrm{MHz}, \mathrm{CDCl}_{3}\right)$

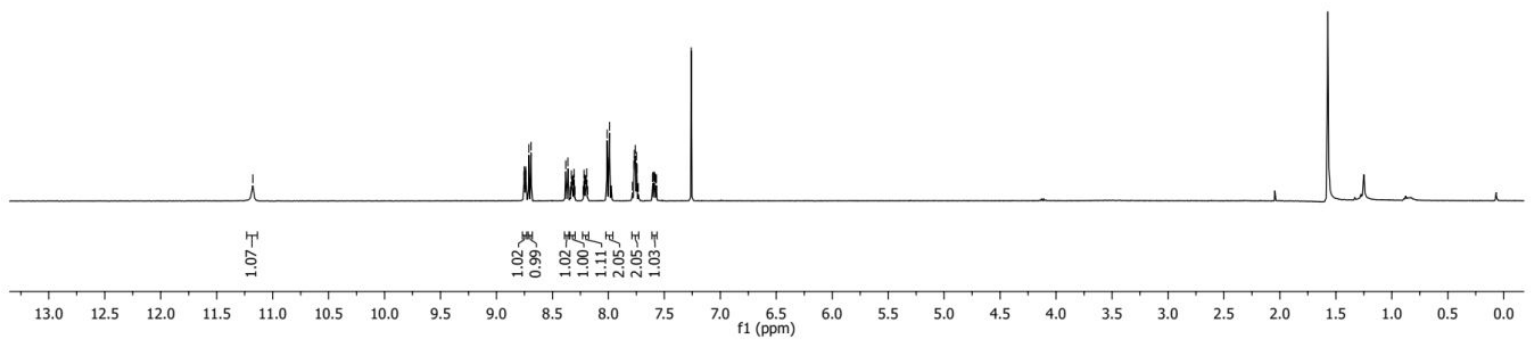

PS-1F-DG-1H

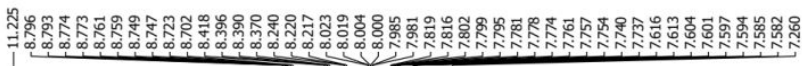

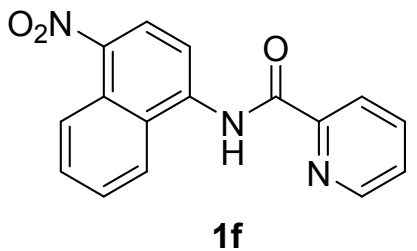

${ }^{1} \mathrm{H}$ NMR $\left(400 \mathrm{MHz}, \mathrm{CDCl}_{3}\right)$

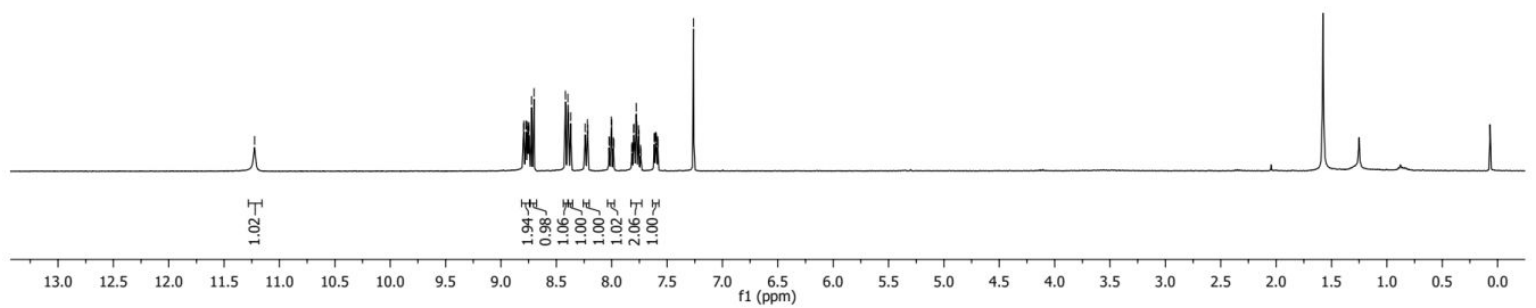




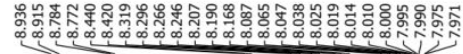

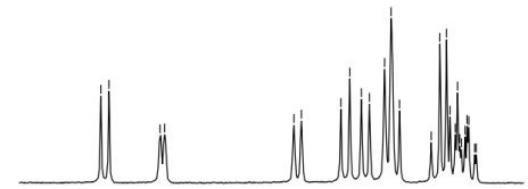

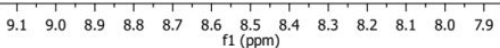<smiles>O=C(Nc1ccc2ccc3cccc4ccc1c2c34)c1ccccn1</smiles>

$1 \mathrm{~g}$

${ }^{1} \mathrm{H} \mathrm{NMR}\left(400 \mathrm{MHz}, \mathrm{CDCl}_{3}\right)$

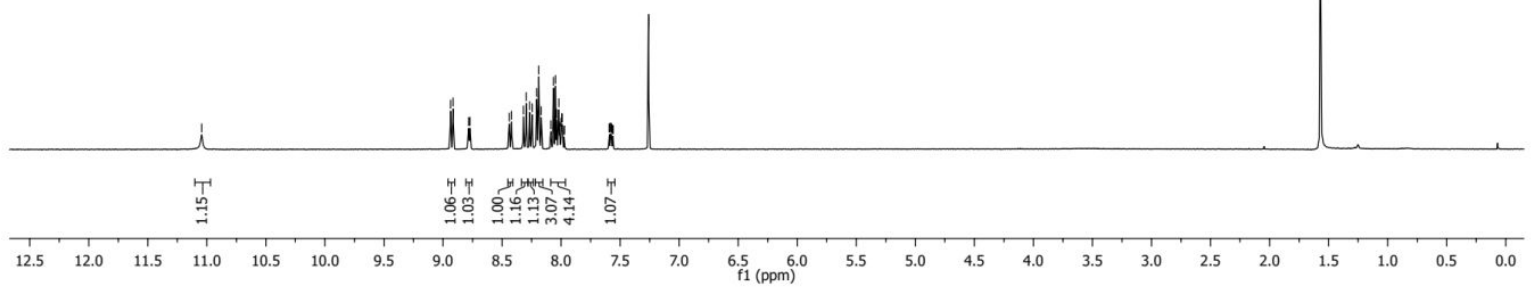

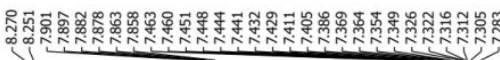

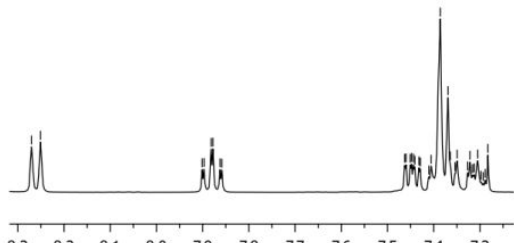<smiles>O=C(NCc1ccccc1)c1ccccn1</smiles>

$1 \mathrm{~h}$

${ }^{1} \mathrm{H}$ NMR $\left(400 \mathrm{MHz}, \mathrm{CDCl}_{3}\right)$

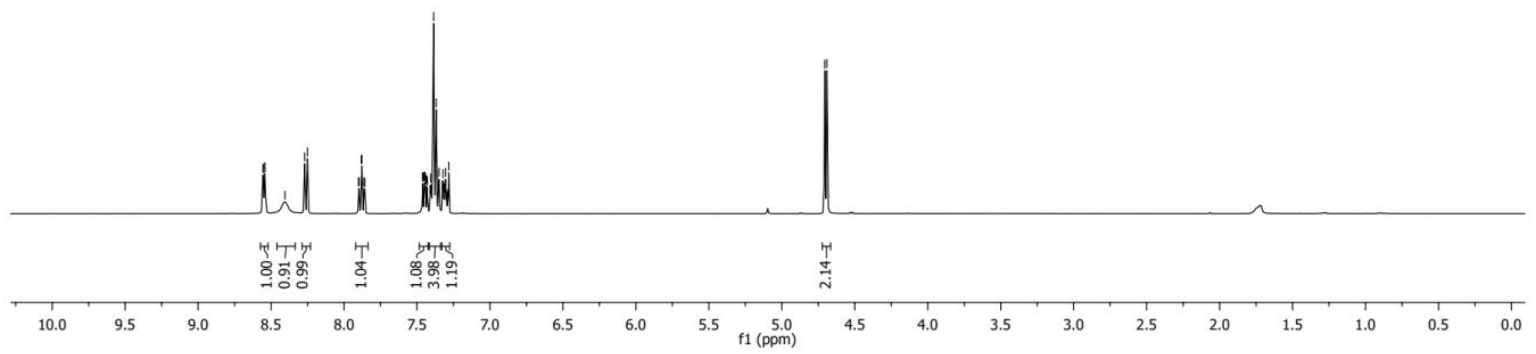




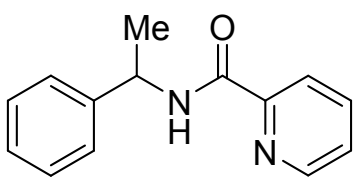

$1 \mathrm{i}$

${ }^{1} \mathrm{H}$ NMR $\left(400 \mathrm{MHz}, \mathrm{CDCl}_{3}\right)$

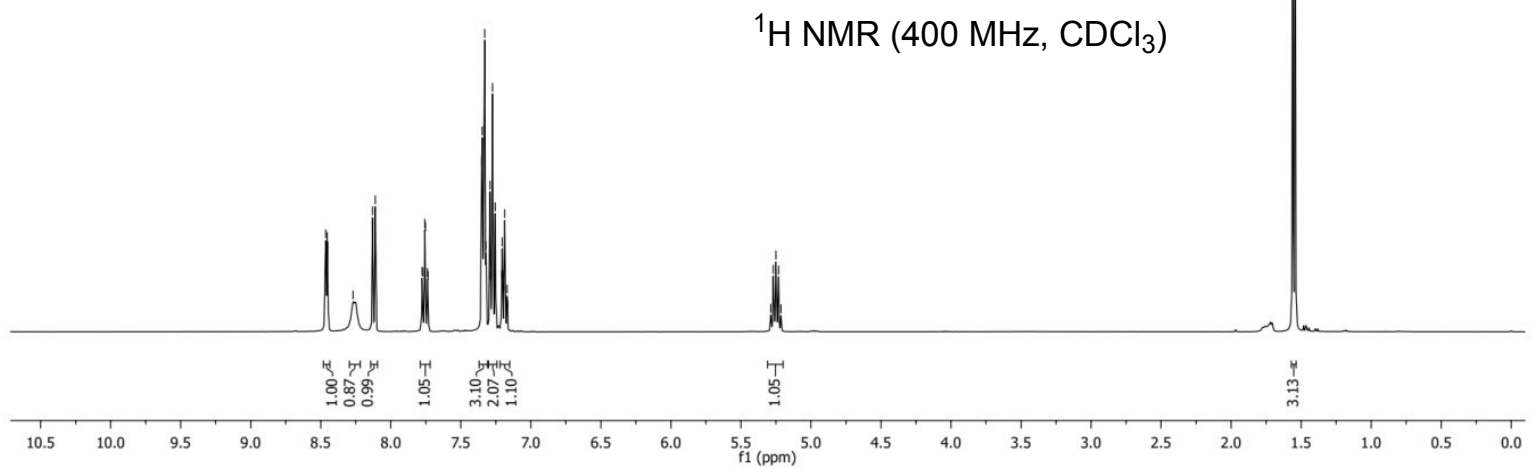

PS-4a-DG-1H<smiles>O=C(Nc1cccc2cccnc12)c1ccccc1</smiles>

$4 a$

${ }^{1} \mathrm{H}$ NMR $\left(400 \mathrm{MHz}, \mathrm{CDCl}_{3}\right)$

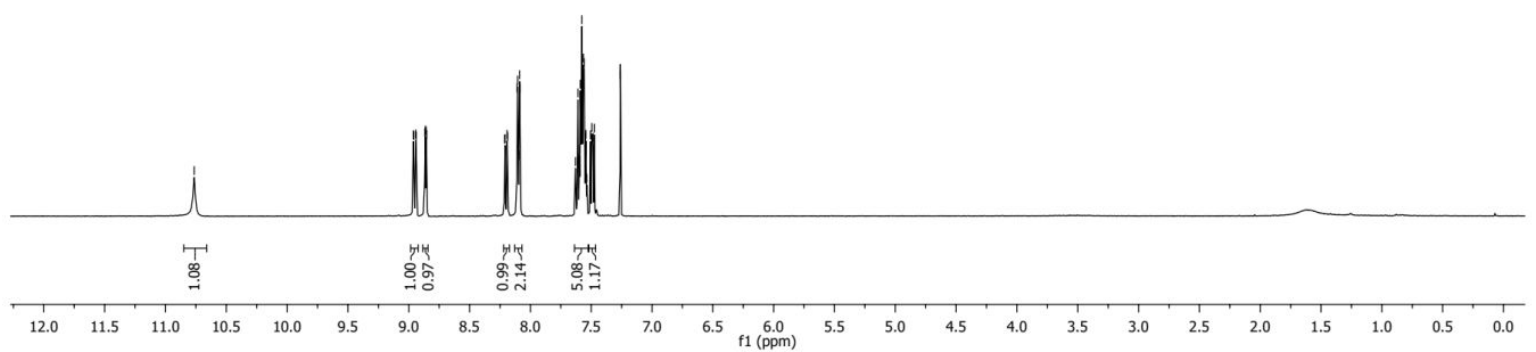


<smiles>Cc1ccccc1C(=O)Nc1cccc2cccnc12</smiles>

4b

${ }^{1} \mathrm{H} \mathrm{NMR}\left(400 \mathrm{MHz}, \mathrm{CDCl}_{3}\right)$

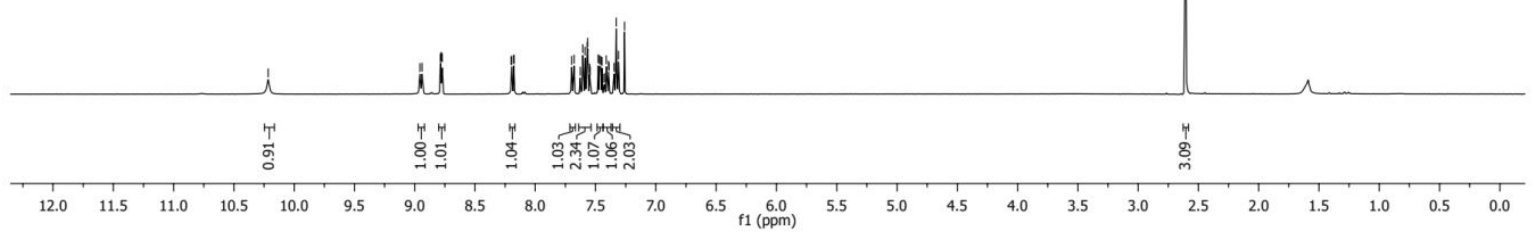

PS-4C-DG-1H

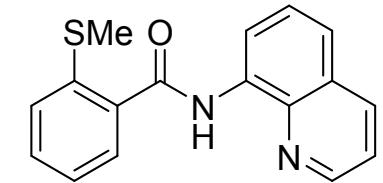

$4 \mathrm{c}$

${ }^{1} \mathrm{H}$ NMR $\left(400 \mathrm{MHz}, \mathrm{CDCl}_{3}\right)$

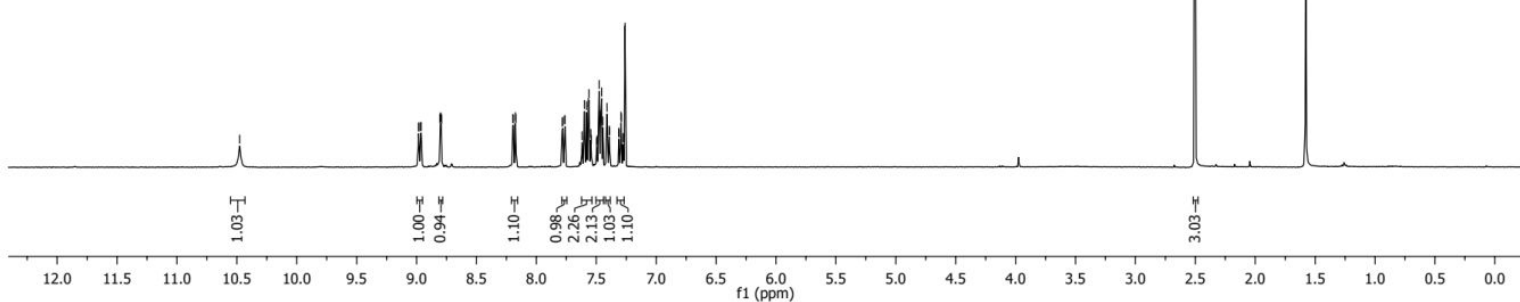


<smiles>O=C(Nc1cccc2cccnc12)c1cccc(Cl)c1</smiles>

4d

${ }^{1} \mathrm{H}$ NMR $\left(400 \mathrm{MHz}, \mathrm{CDCl}_{3}\right)$

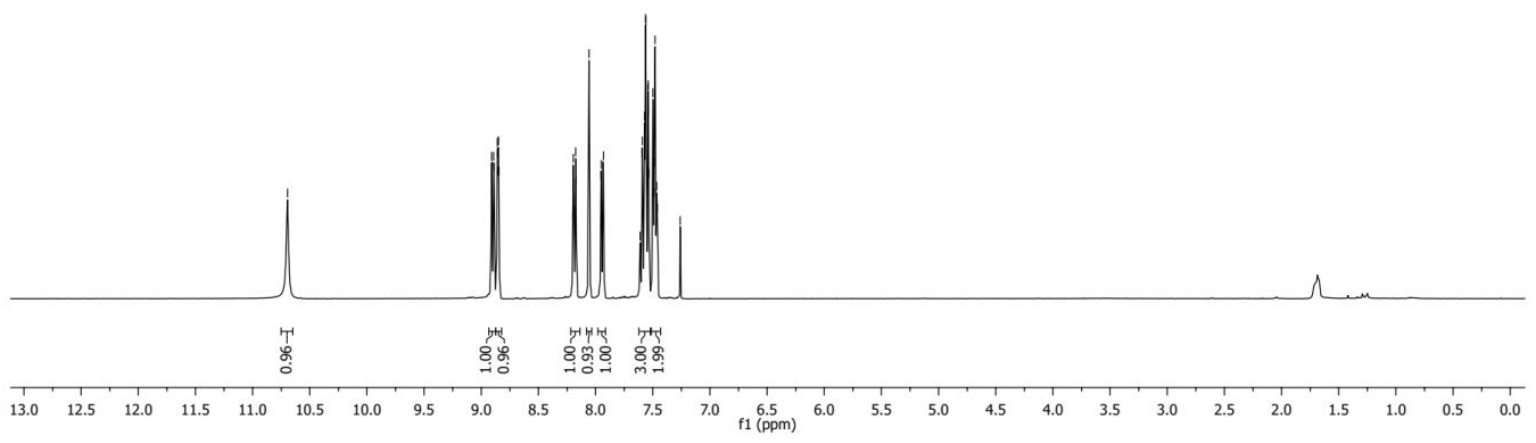

PS-4e-DG-1H

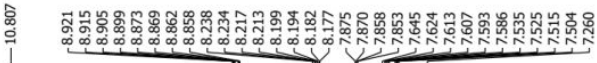

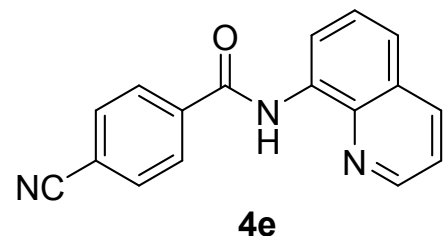

${ }^{1} \mathrm{H}$ NMR $\left(400 \mathrm{MHz}, \mathrm{CDCl}_{3}\right)$

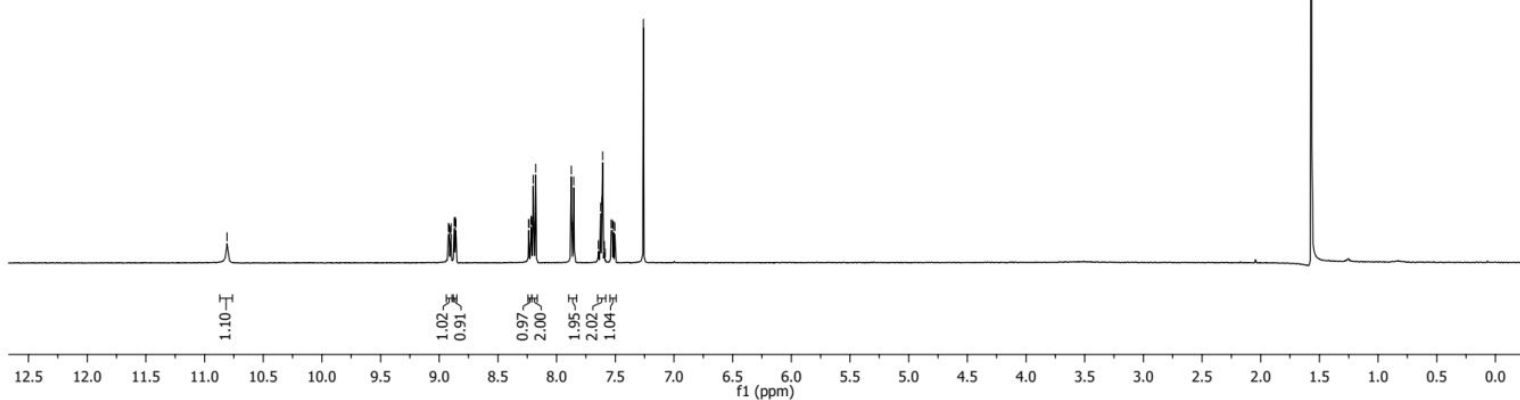




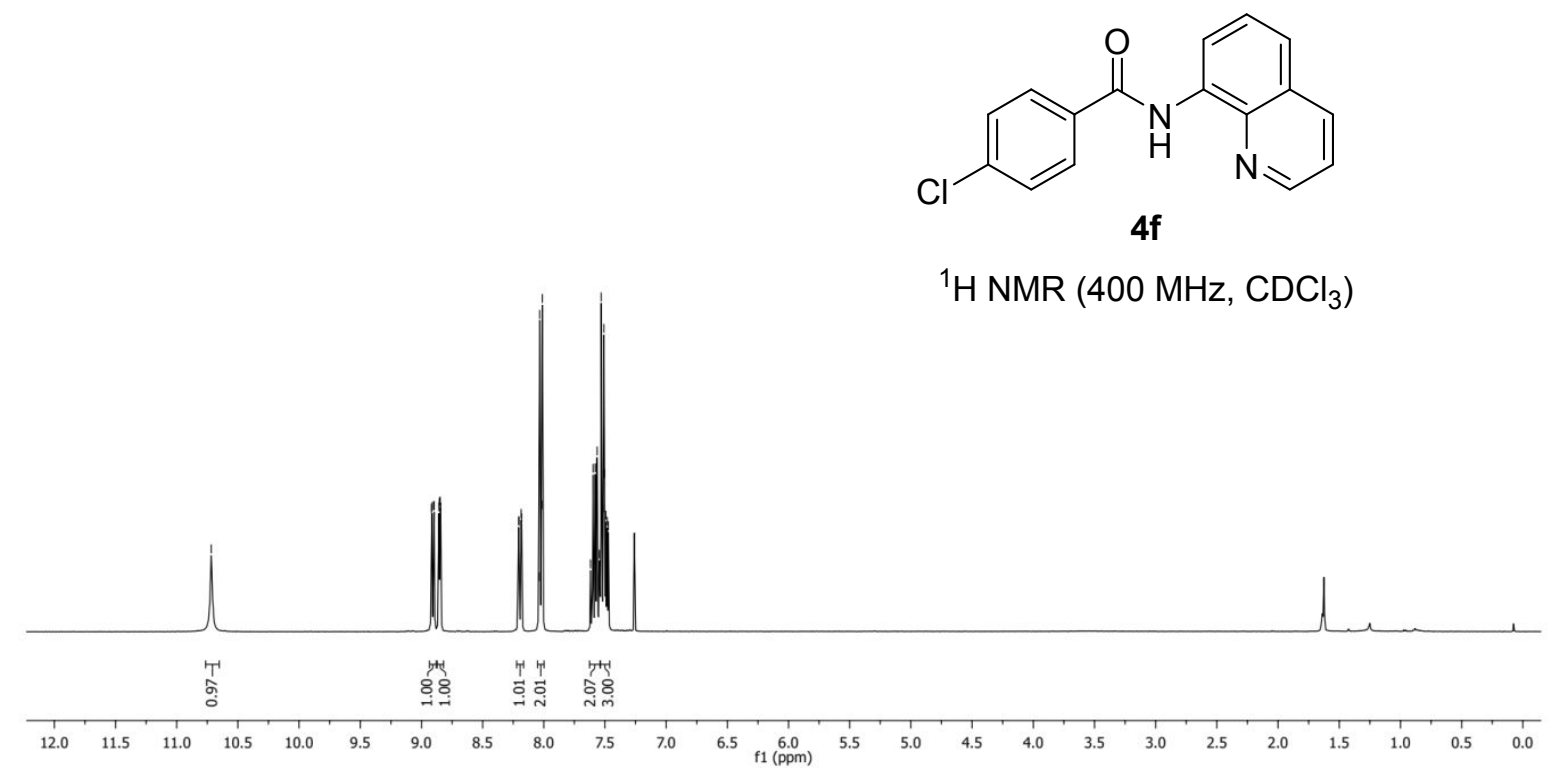

PS-4g-DG-1H

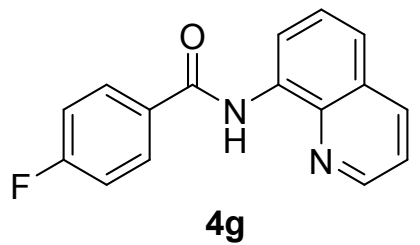

${ }^{1} \mathrm{H}$ NMR $\left(400 \mathrm{MHz}, \mathrm{CDCl}_{3}\right)$

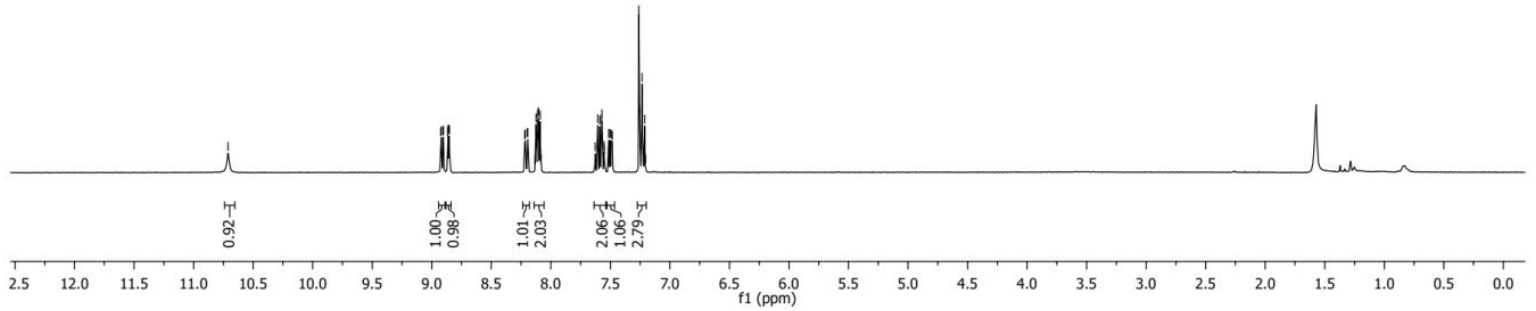




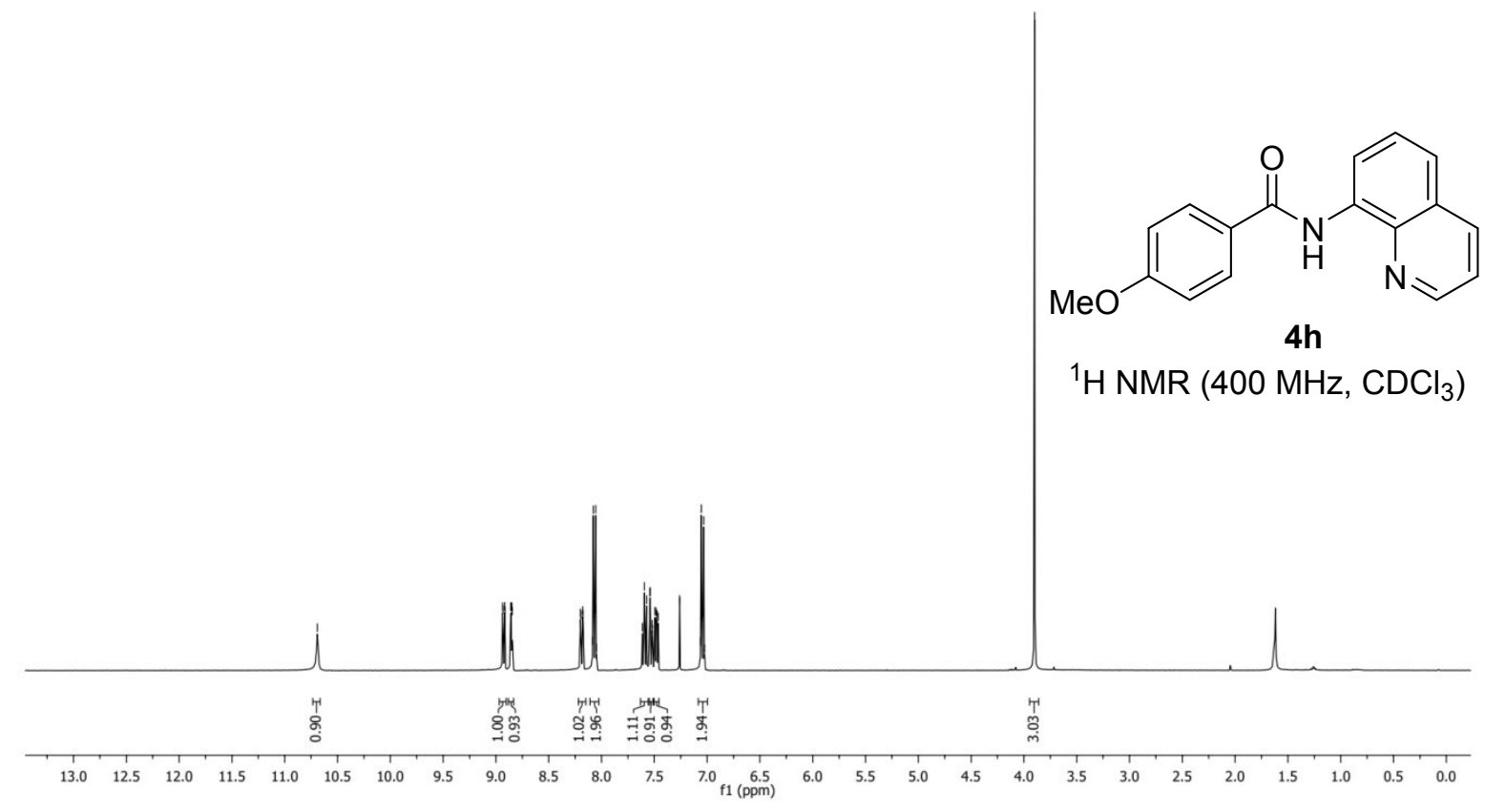

PS-4I-DG-1H

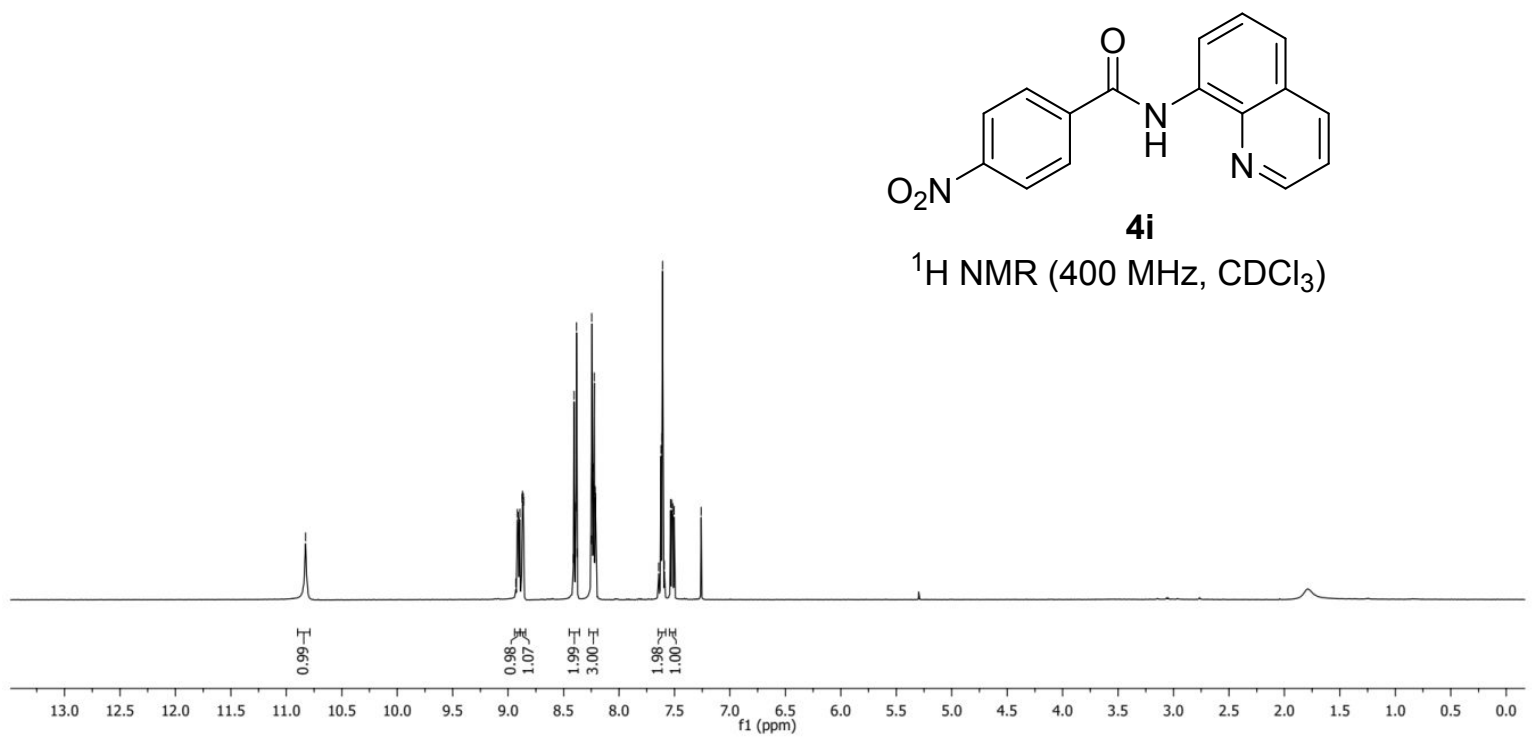



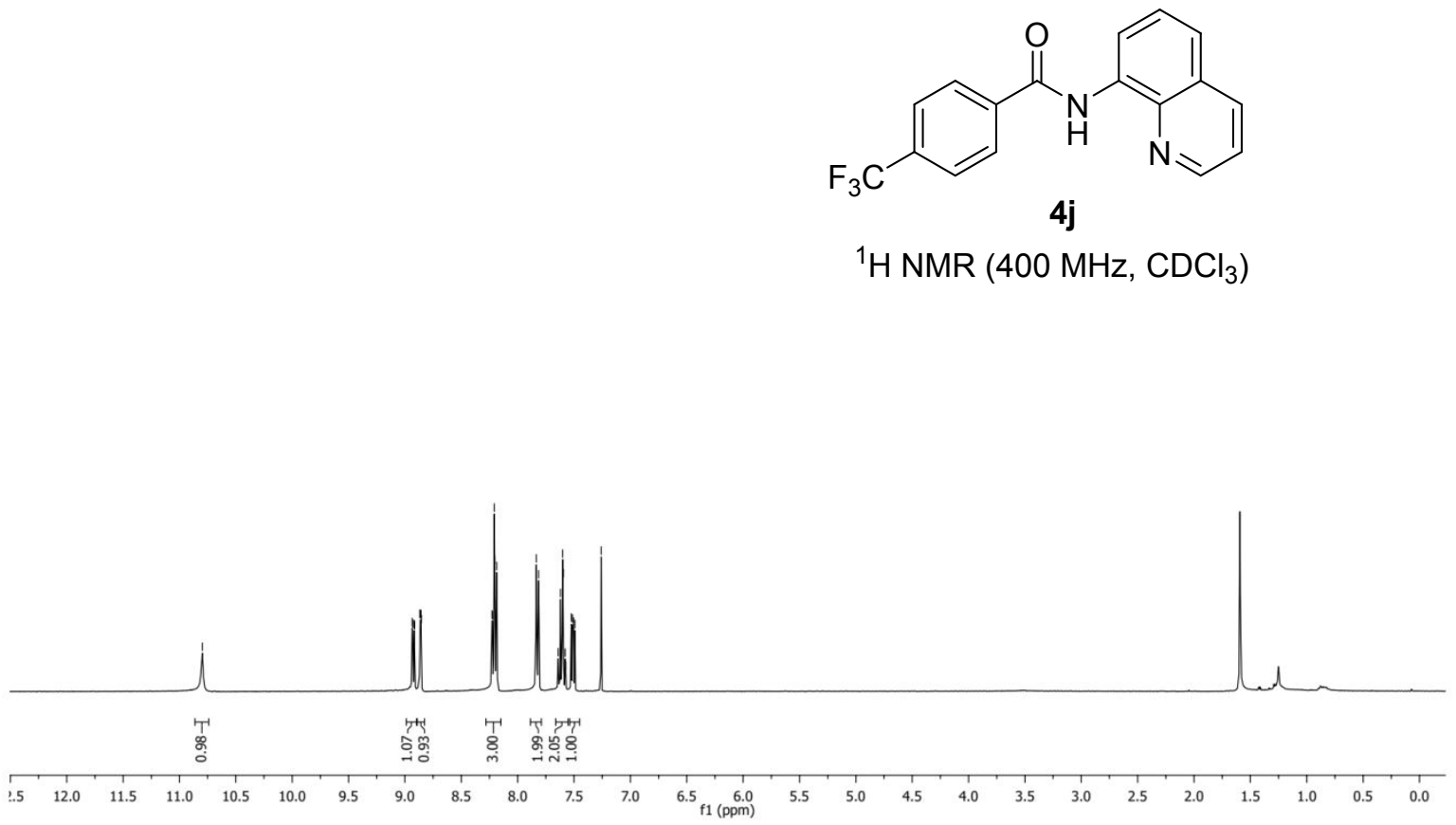

PS-4K-DG-1H

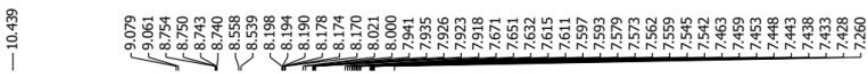<smiles>O=C(Nc1cccc2cccnc12)c1cccc2ccccc12</smiles>

4k

${ }^{1} \mathrm{H} \mathrm{NMR}\left(400 \mathrm{MHz}, \mathrm{CDCl}_{3}\right)$

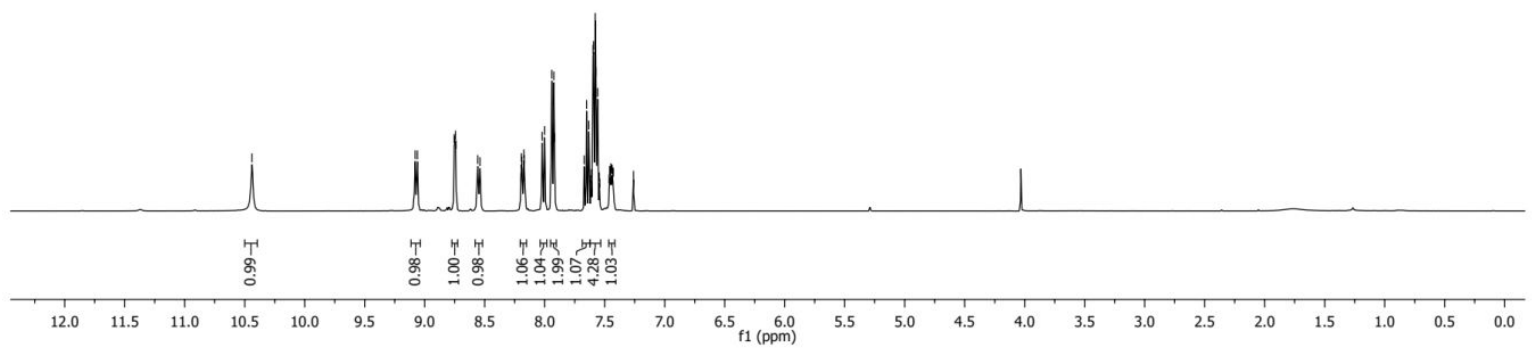




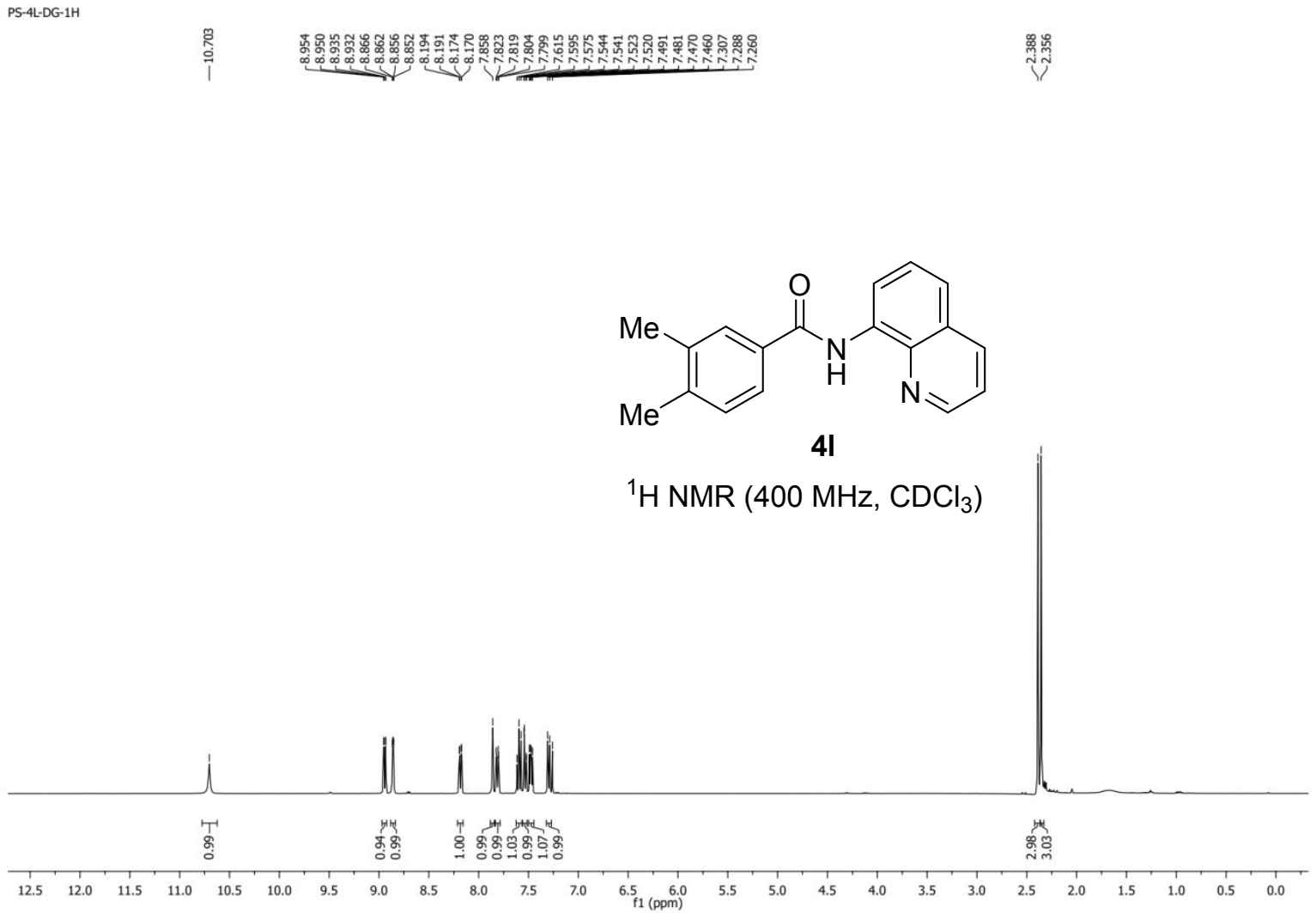

CI-Me-AQ-DG-1H

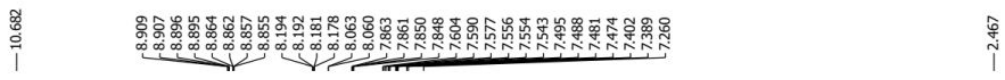

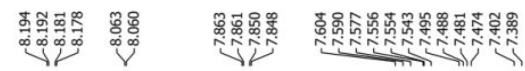
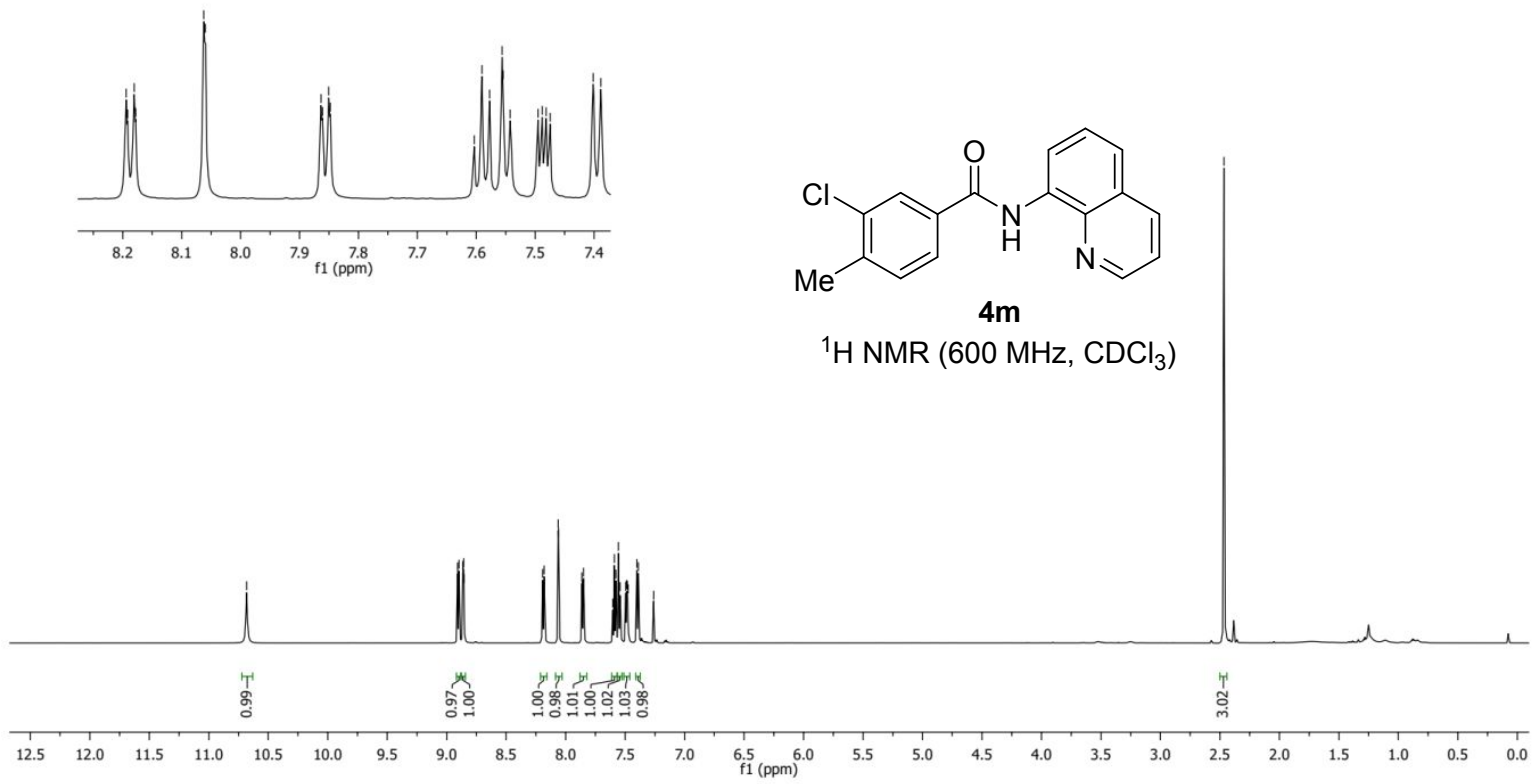


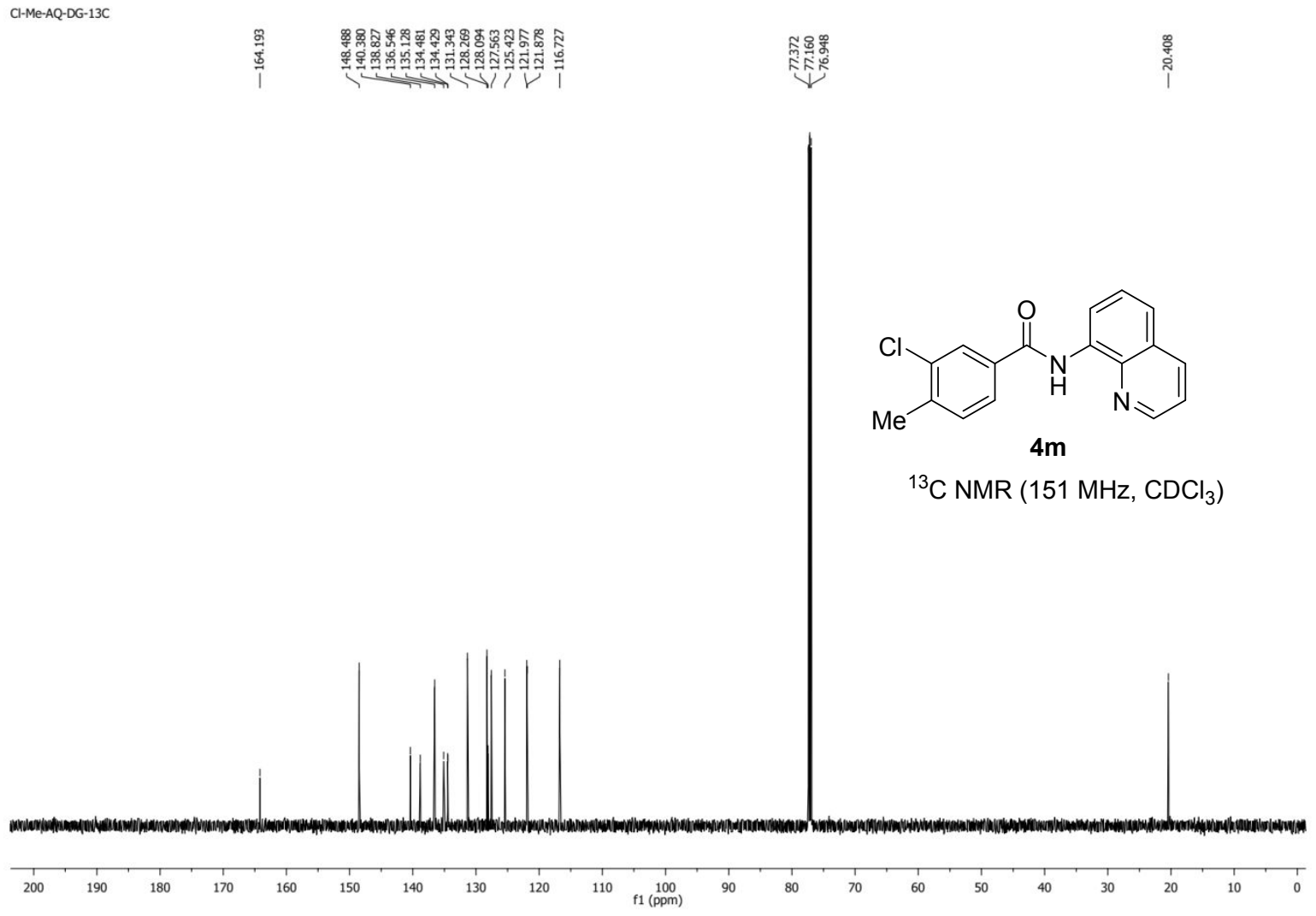

PS-4N-DG-1H
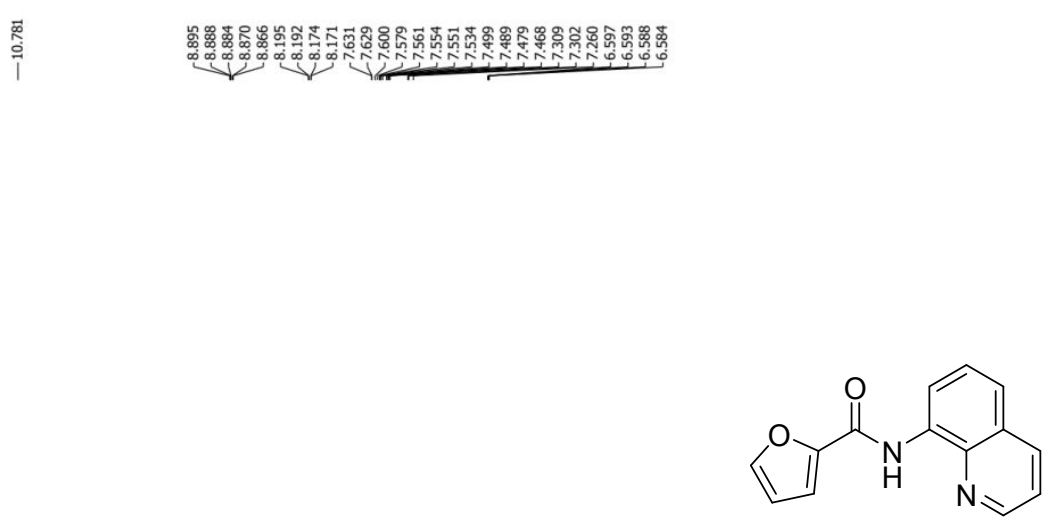

$4 n$

${ }^{1} \mathrm{H}$ NMR (400 MHz, $\left.\mathrm{CDCl}_{3}\right)$

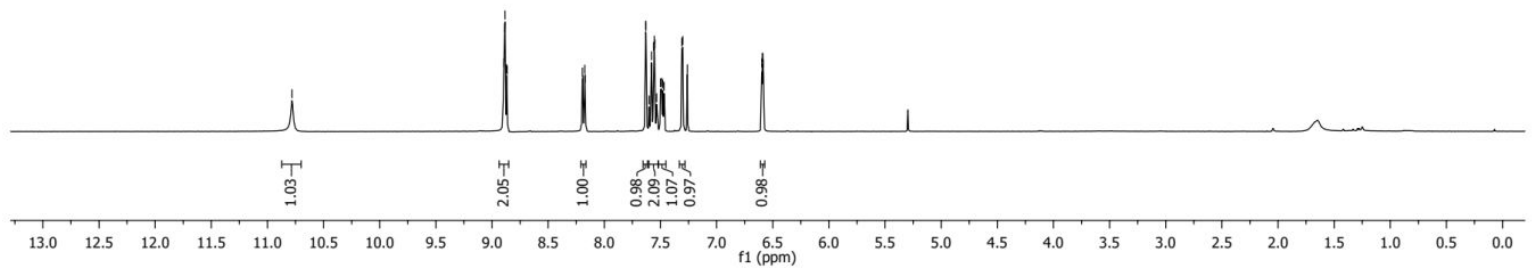




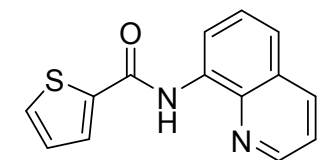

4o

${ }^{1} \mathrm{H}$ NMR $\left(400 \mathrm{MHz}, \mathrm{CDCl}_{3}\right)$

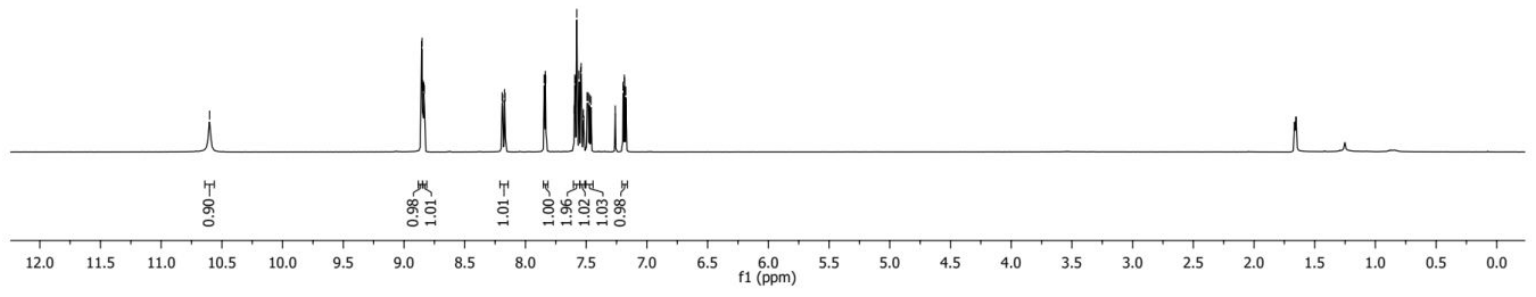

PS-4P-DG-1H<smiles>O=C(Nc1cccc2cccnc12)c1ccncc1</smiles>

$4 p$

${ }^{1} \mathrm{H}$ NMR $\left(400 \mathrm{MHz}, \mathrm{CDCl}_{3}\right)$

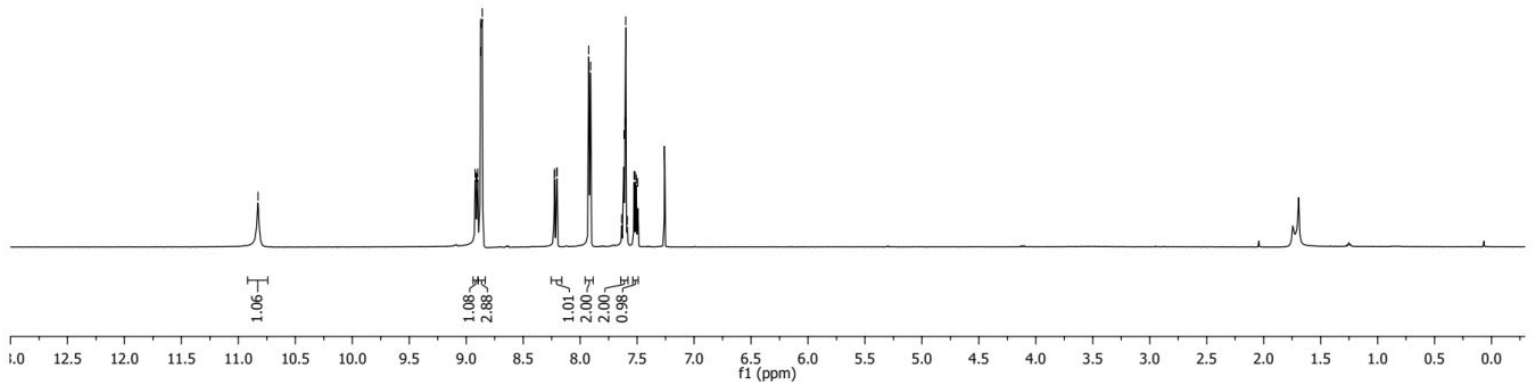




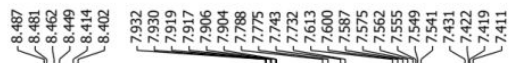

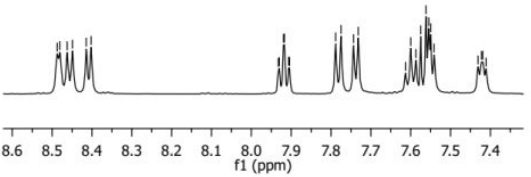

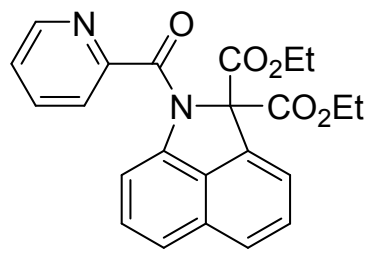

3a

${ }^{1} \mathrm{H}$ NMR $\left(600 \mathrm{MHz}, \mathrm{CDCl}_{3}\right)$
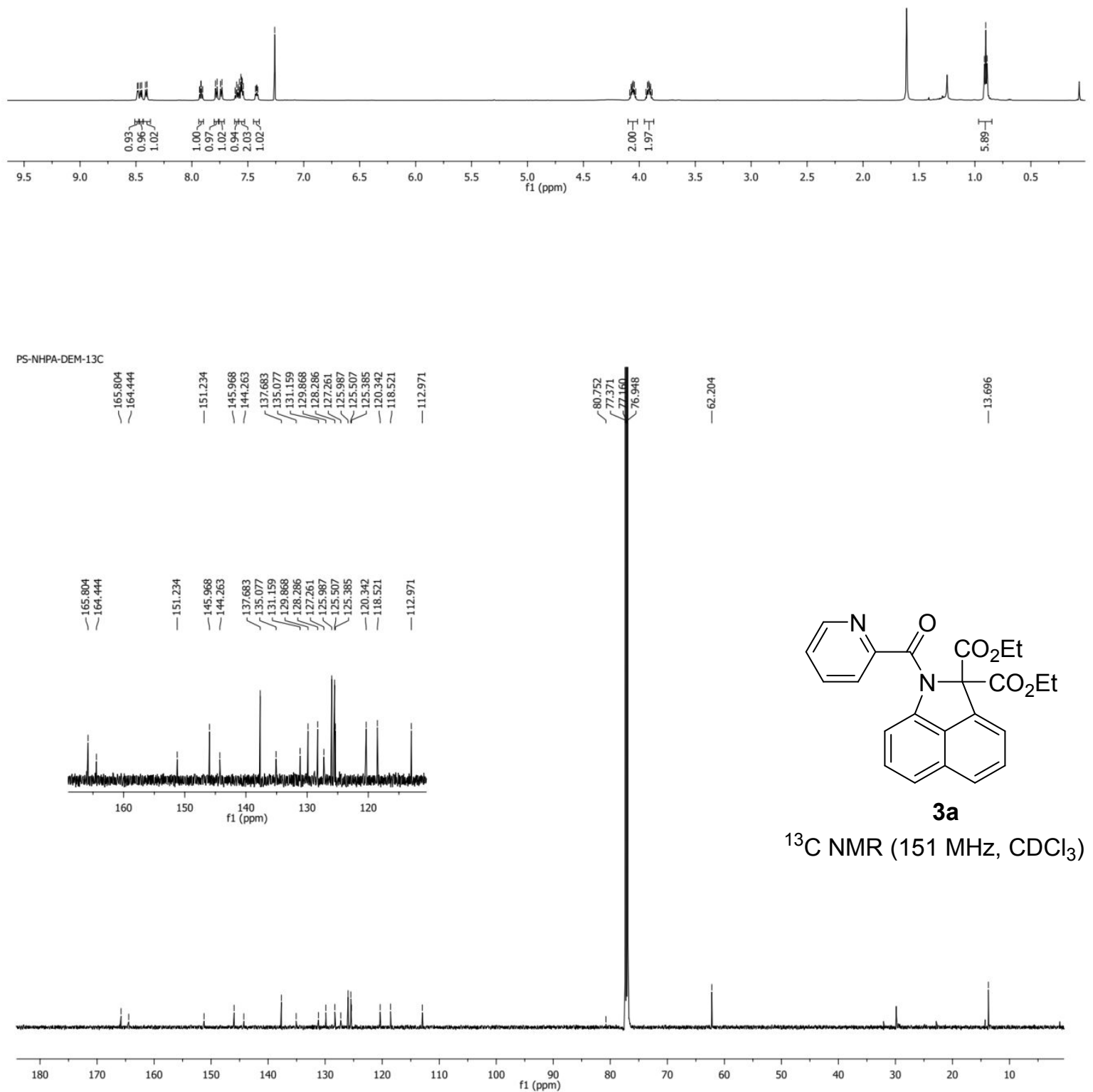


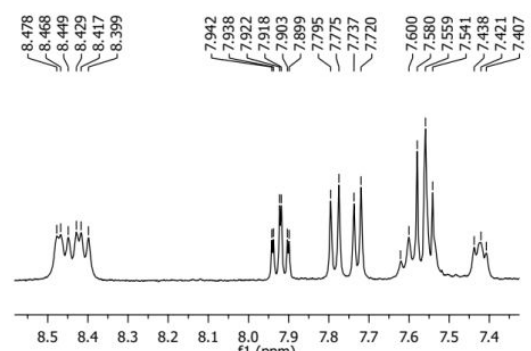

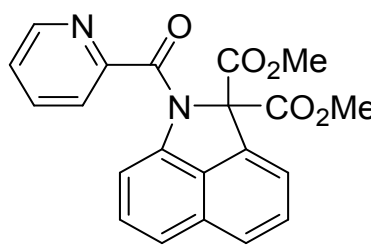

3b

${ }^{1} \mathrm{H} \mathrm{NMR}\left(400 \mathrm{MHz}, \mathrm{CDCl}_{3}\right)$
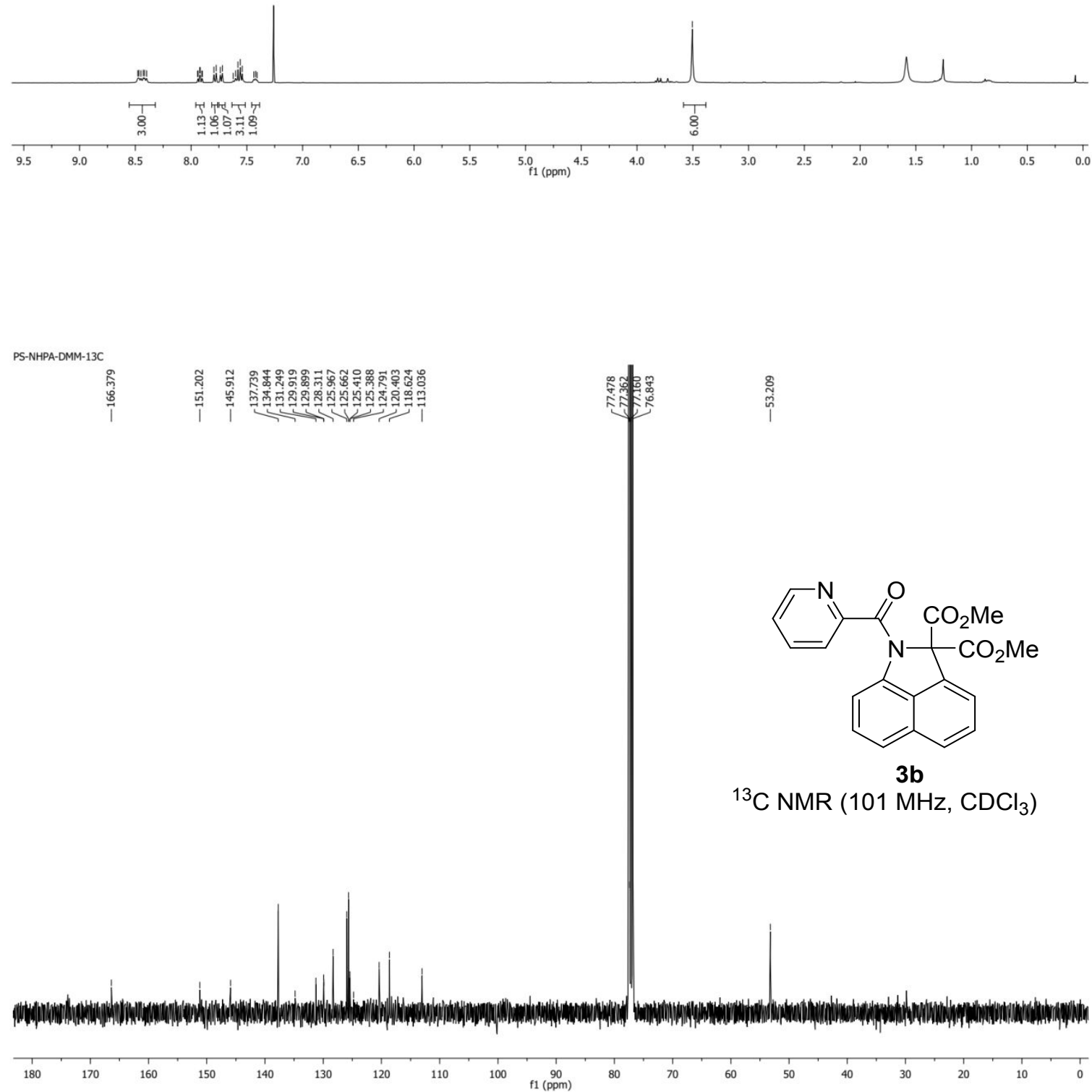

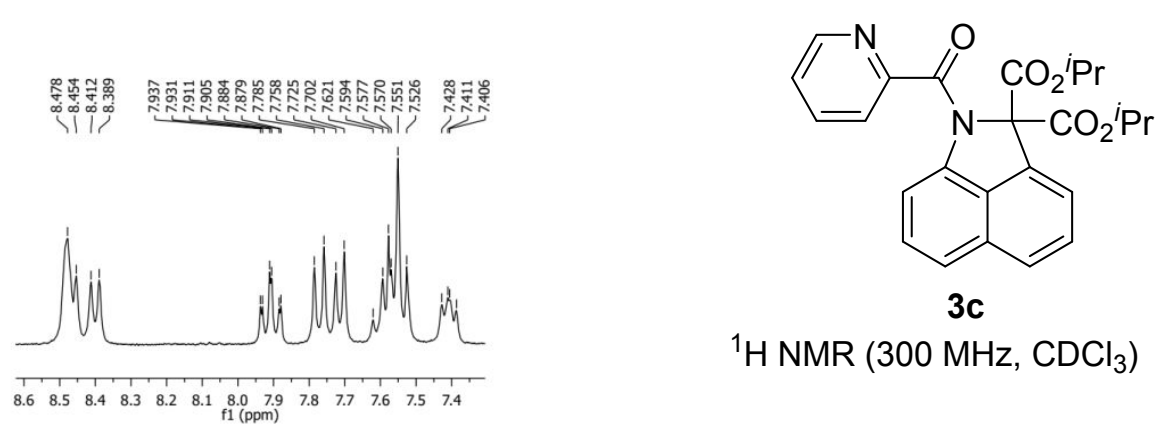

${ }^{1} \mathrm{H}$ NMR $\left(300 \mathrm{MHz}, \mathrm{CDCl}_{3}\right)$
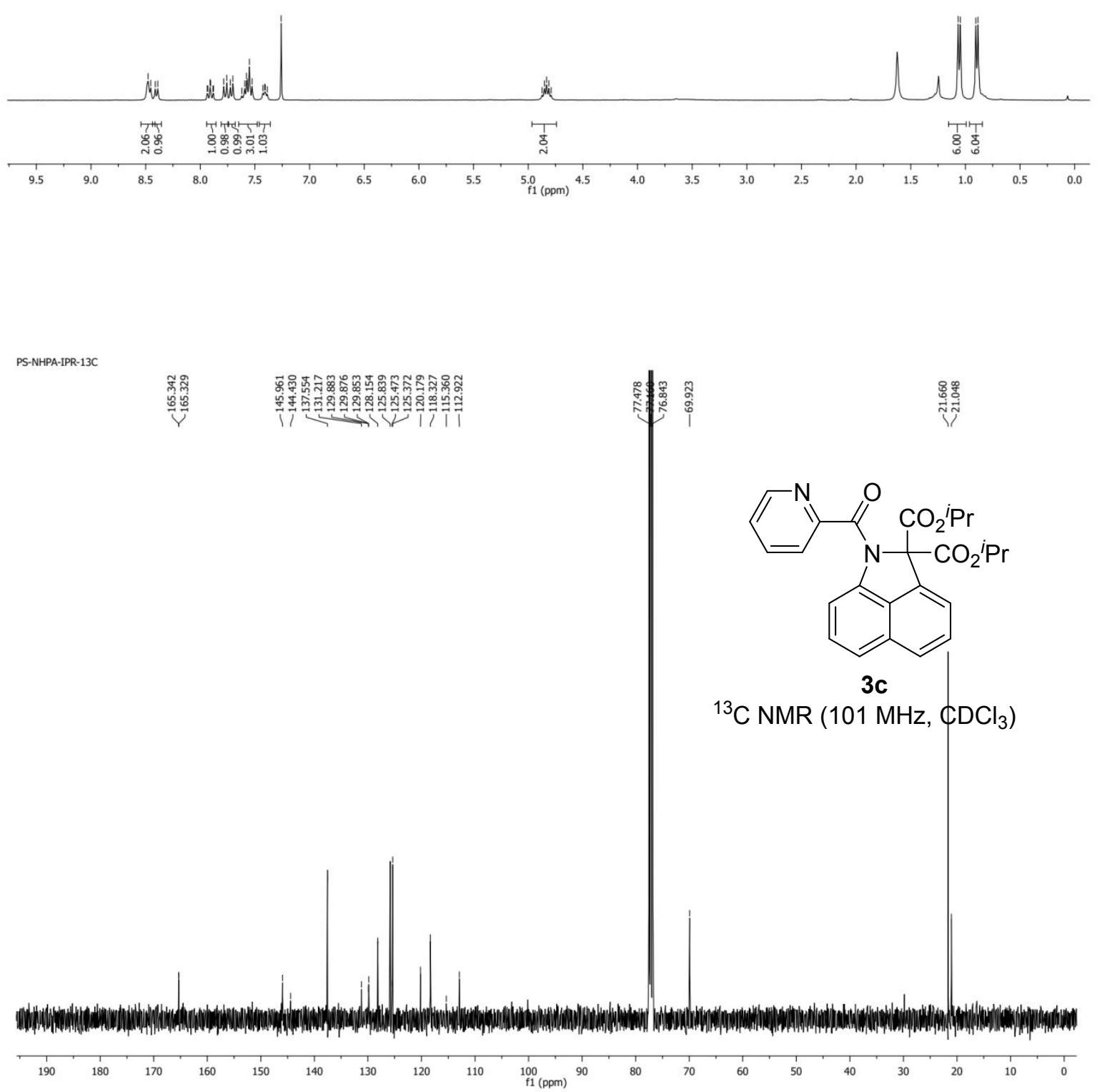


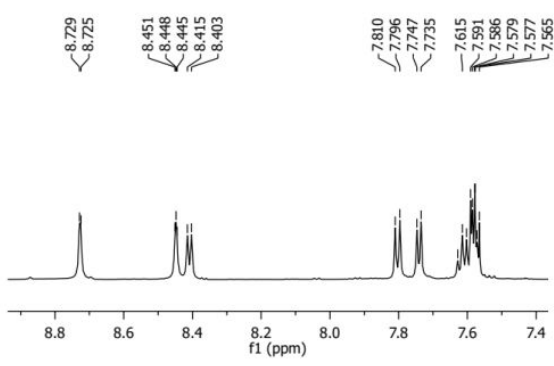

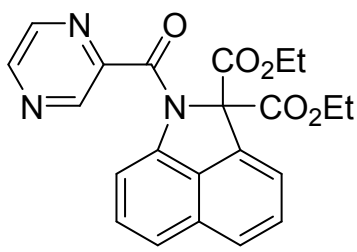

3d

${ }^{1} \mathrm{H}$ NMR $\left(600 \mathrm{MHz}, \mathrm{CDCl}_{3}\right)$

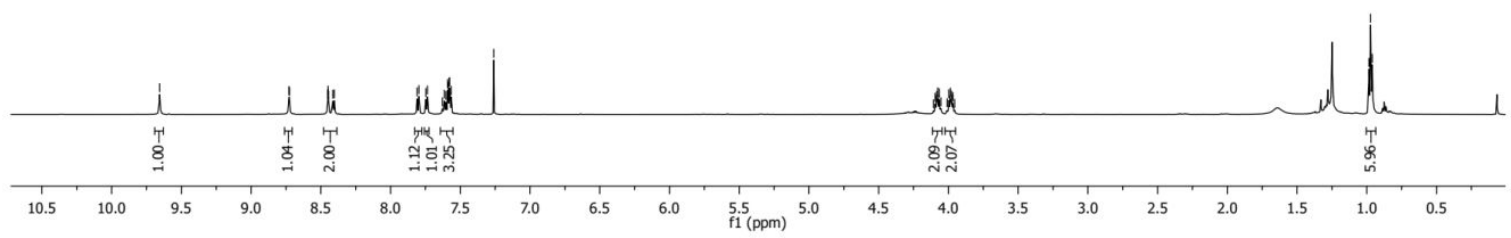

PS-PIPZ-PA-13C/10

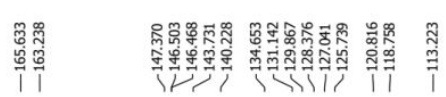

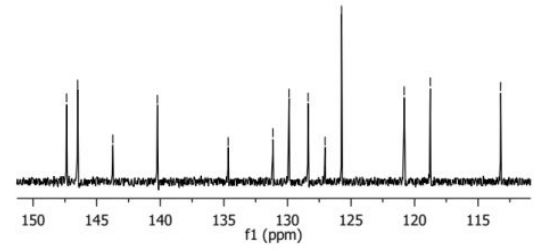

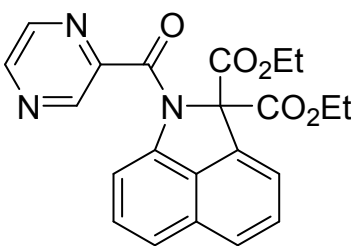

3d

${ }^{13} \mathrm{C}$ NMR (151 MHz, $\left.\mathrm{CDCl}_{3}\right)$ 

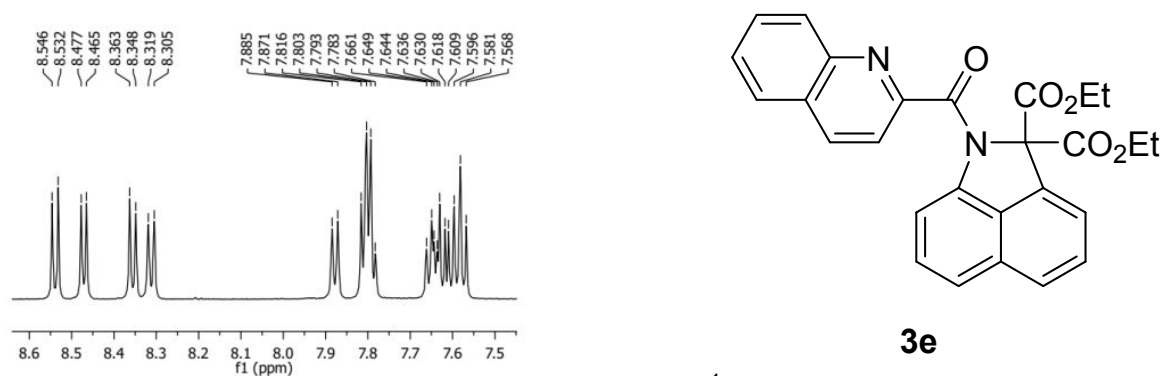

$3 e$

${ }^{1} \mathrm{H}$ NMR $\left(600 \mathrm{MHz}, \mathrm{CDCl}_{3}\right)$

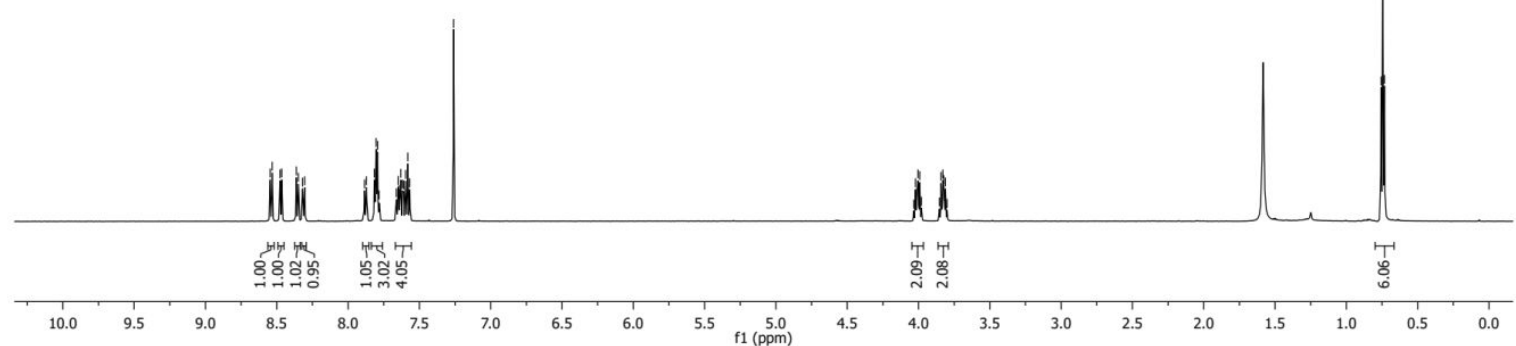

PS-QA-PA-13C

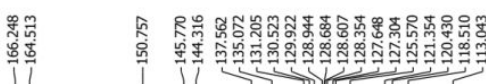

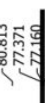

$\underset{\text { กิ }}{\text { I }}$

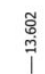

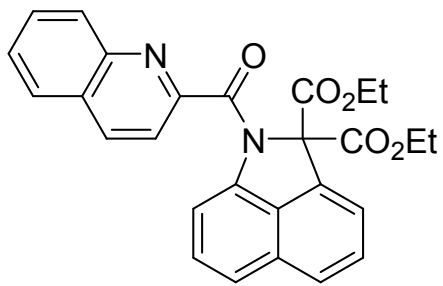

$3 e$

${ }^{13} \mathrm{C}$ NMR $\left(151 \mathrm{MHz}, \mathrm{CDCl}_{3}\right)$

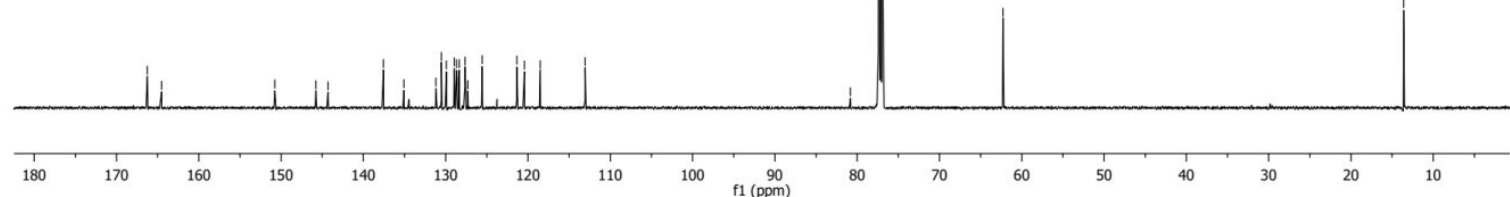




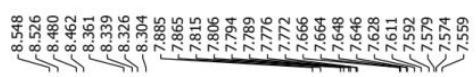

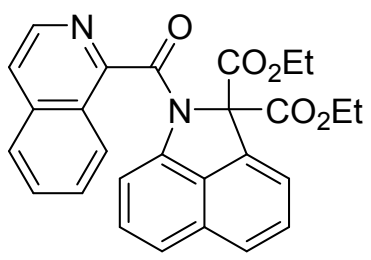

iin jim

whenth

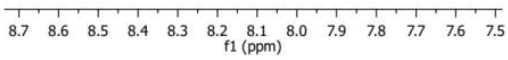

$3 f$

${ }^{1} \mathrm{H}$ NMR $\left(400 \mathrm{MHz}, \mathrm{CDCl}_{3}\right)$
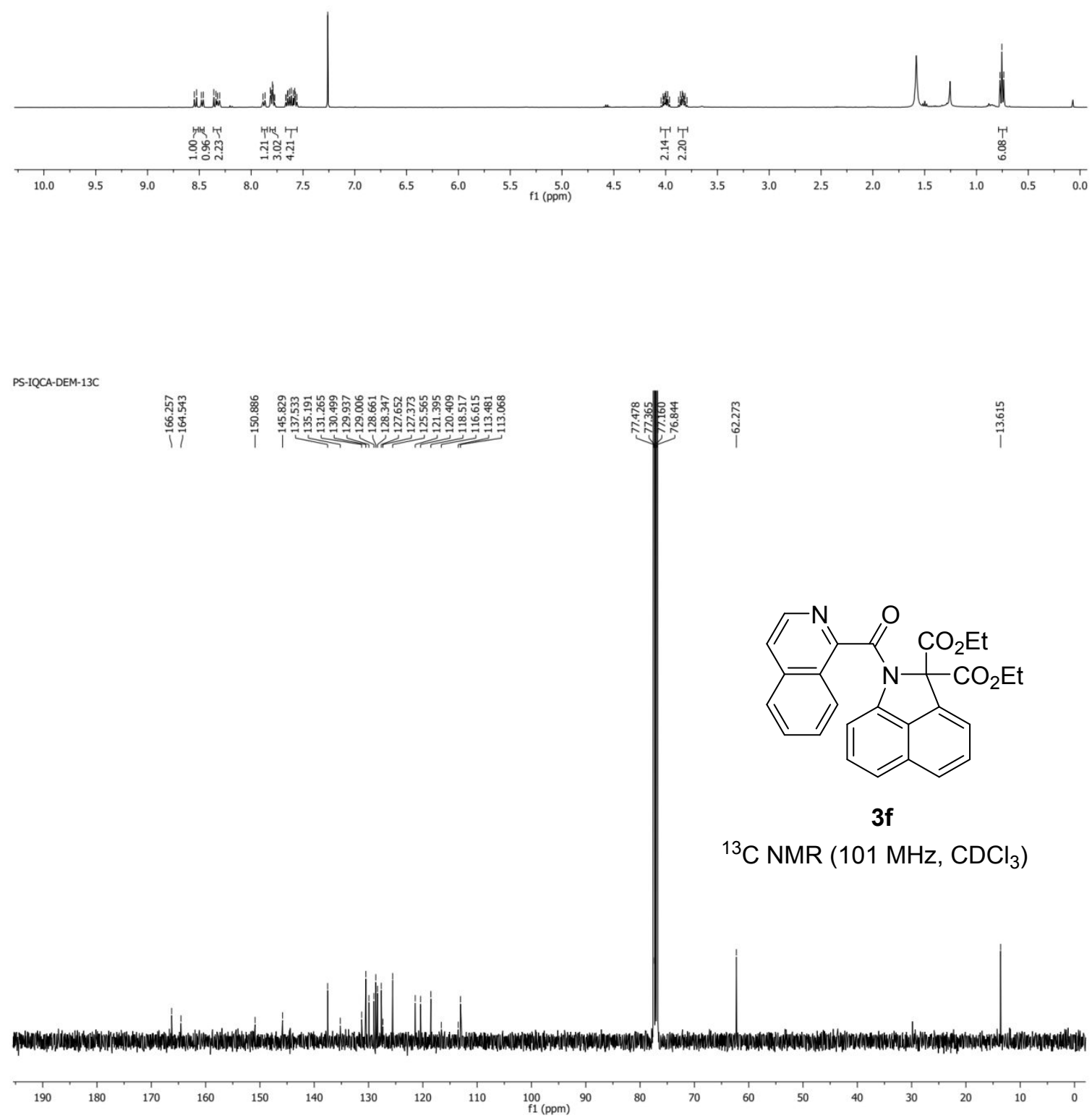


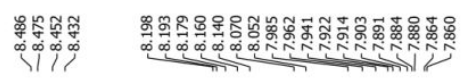

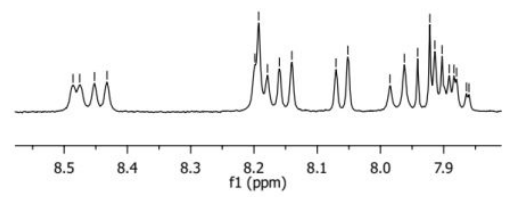

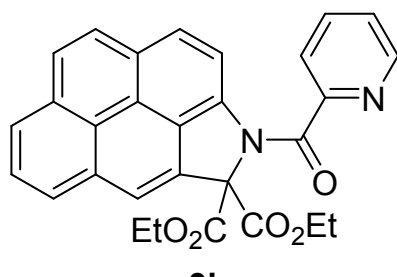

$3 \mathbf{i}$

${ }^{1} \mathrm{H}$ NMR $\left(400 \mathrm{MHz}, \mathrm{CDCl}_{3}\right)$

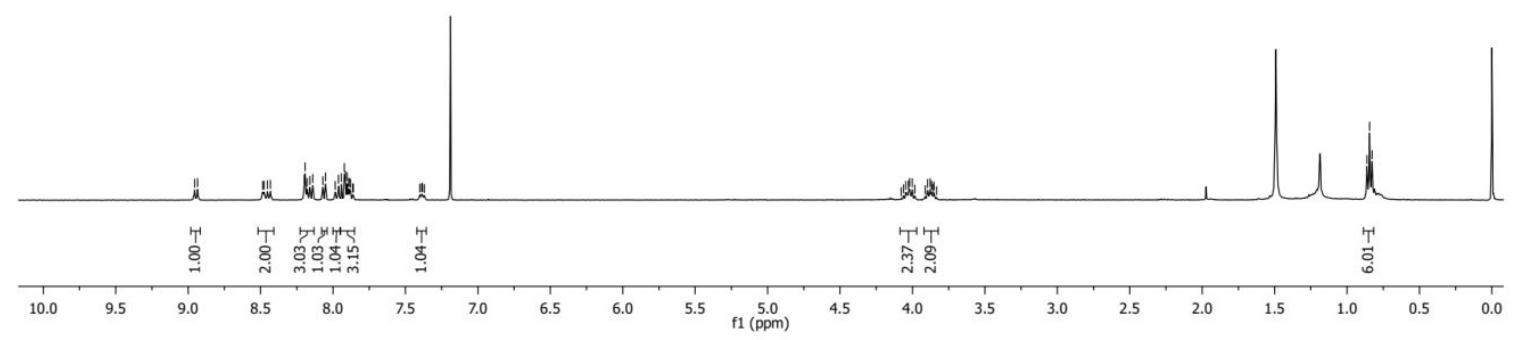

PS-AMPY-DEM-13C<smiles></smiles>

$3 \mathbf{i}$

${ }^{13} \mathrm{C}$ NMR $\left(101 \mathrm{MHz}, \mathrm{CDCl}_{3}\right)$ 


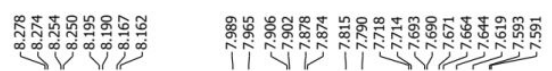

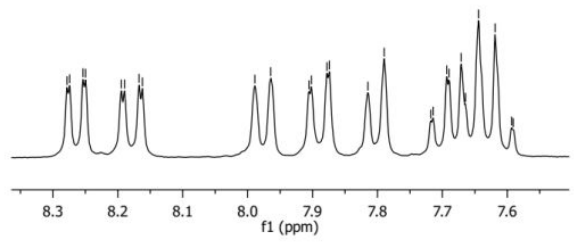<smiles>CCOC(=O)C1(C(=O)OCC)c2ccccc2C(=O)N1c1cccc2cccnc12</smiles>

$5 a$

${ }^{1} \mathrm{H}$ NMR $\left(300 \mathrm{MHz}, \mathrm{CDCl}_{3}\right)$

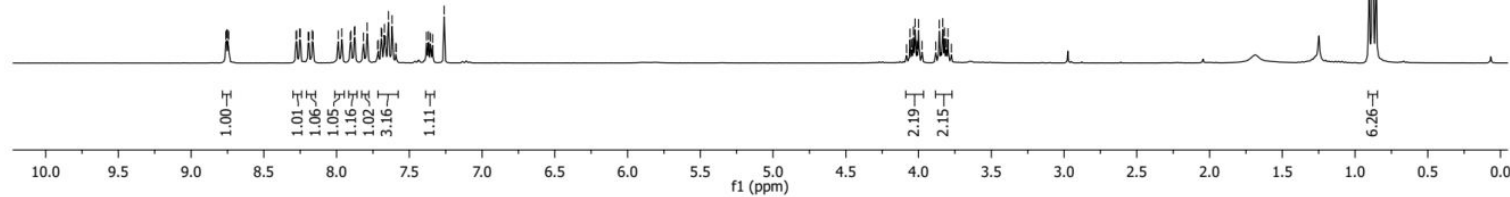

AQ-DEM-13C

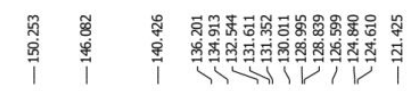
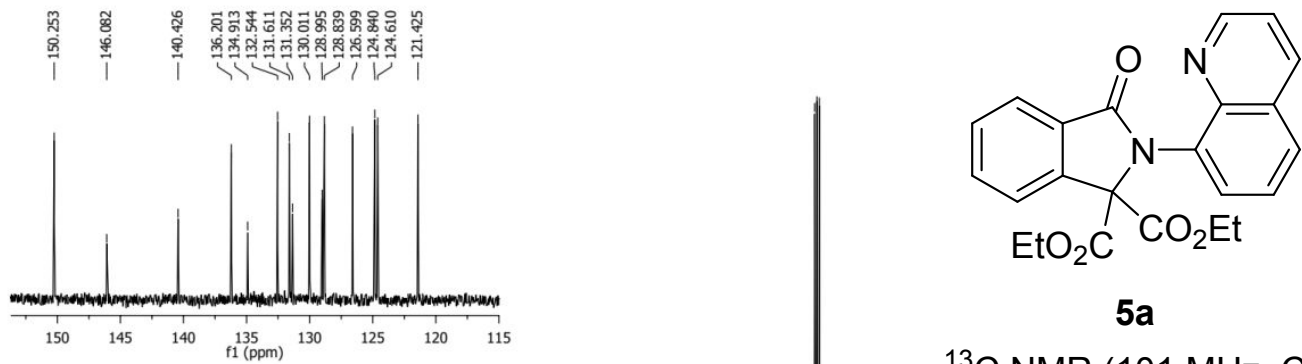

$5 a$

${ }^{13} \mathrm{C} \mathrm{NMR}\left(101 \mathrm{MHz}, \mathrm{CDCl}_{3}\right)$

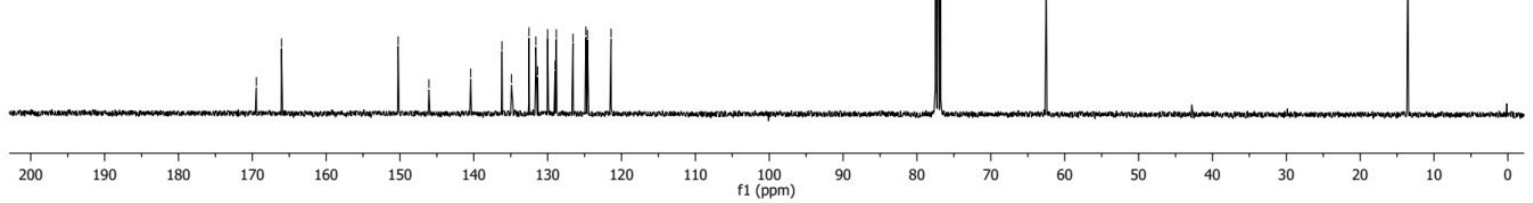




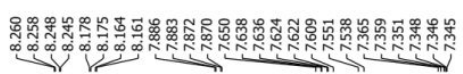

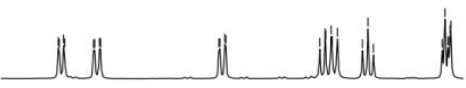

$\begin{array}{llllllllllllll}8.3 & 8.2 & 8.1 & 8.0 & 7.9 & 7.8 & 7 & 1 & 1 & 7.7 & 7.6 & 7.5 & 7.4\end{array}$<smiles>CCOC(=O)C1(C(=O)OCC)c2cccc(C)c2C(=O)N1c1cccc2cccnc12</smiles>

$5 b$

${ }^{1} \mathrm{H}$ NMR $\left(600 \mathrm{MHz}, \mathrm{CDCl}_{3}\right)$

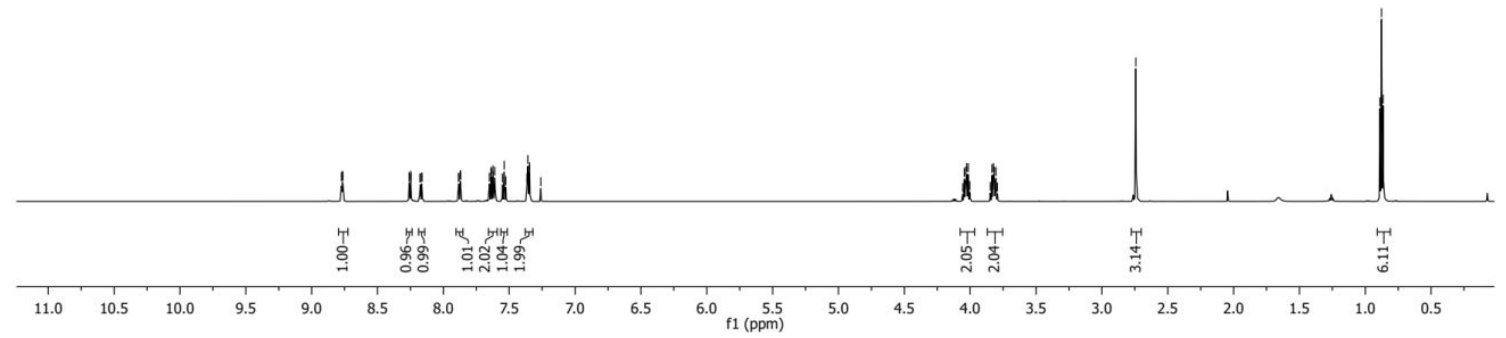

PS-AQ-2ME-13C

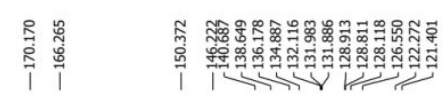

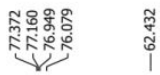

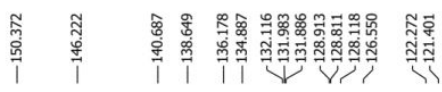

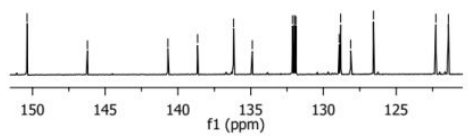

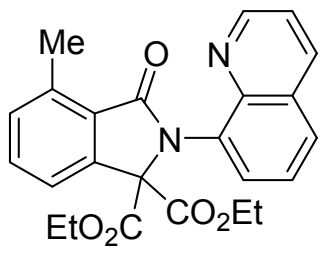

$5 b$

${ }^{13} \mathrm{C}$ NMR $\left(151 \mathrm{MHz}, \mathrm{CDCl}_{3}\right)$

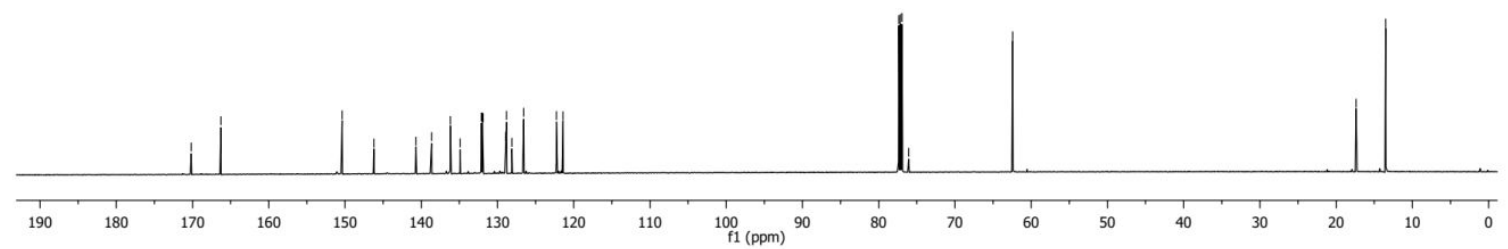




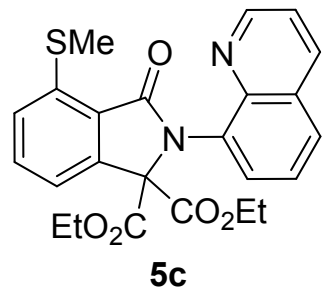

${ }^{1} \mathrm{H}$ NMR $\left(400 \mathrm{MHz}, \mathrm{CDCl}_{3}\right)$
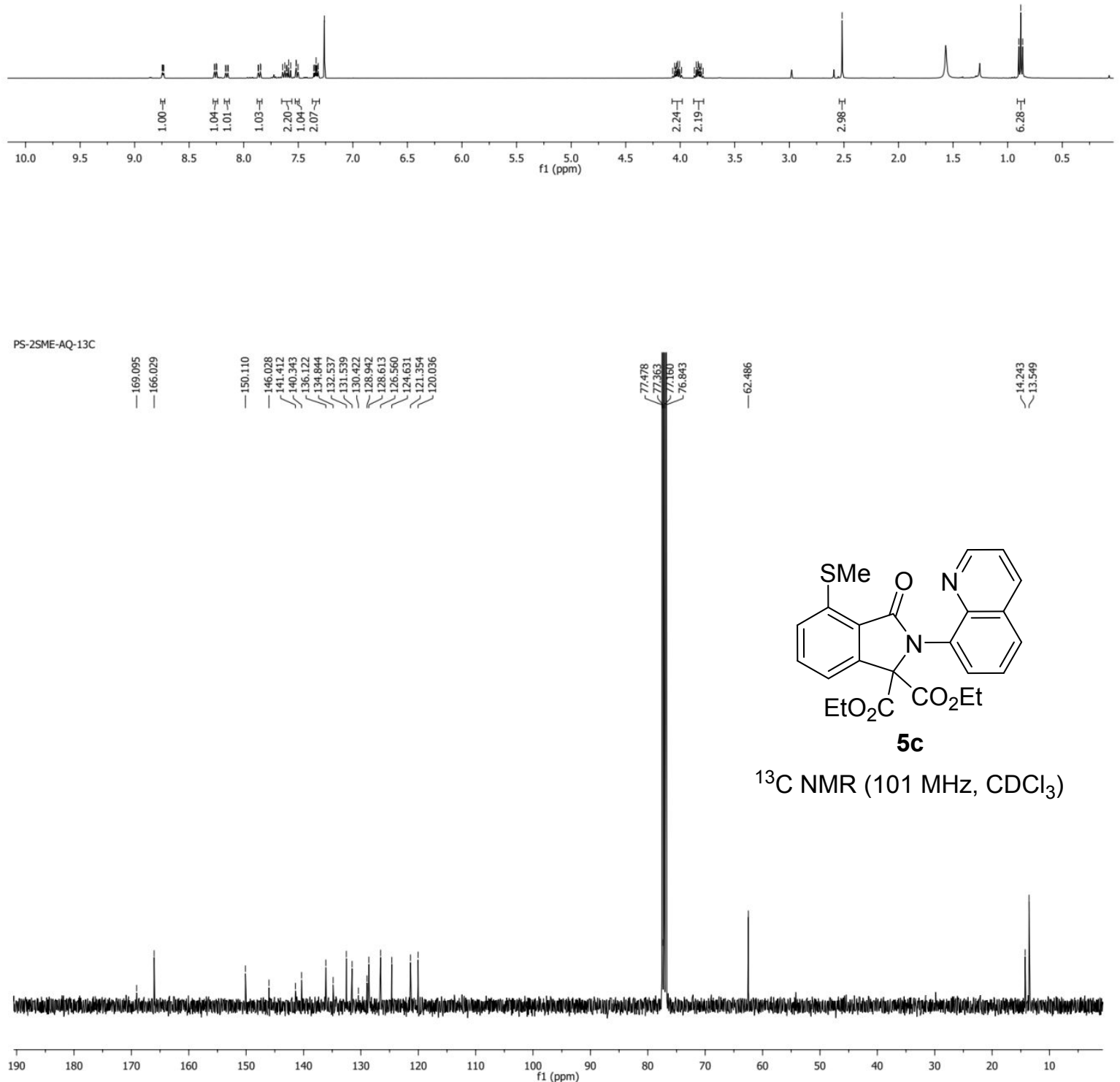


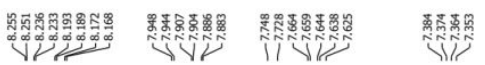
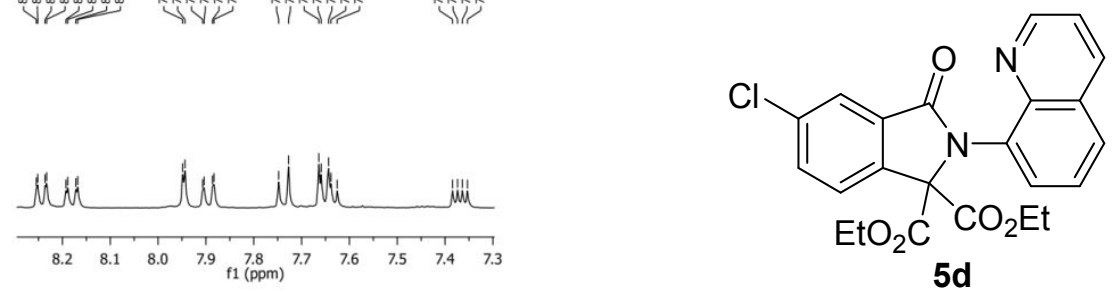

${ }^{1} \mathrm{H}$ NMR $\left(400 \mathrm{MHz}, \mathrm{CDCl}_{3}\right)$
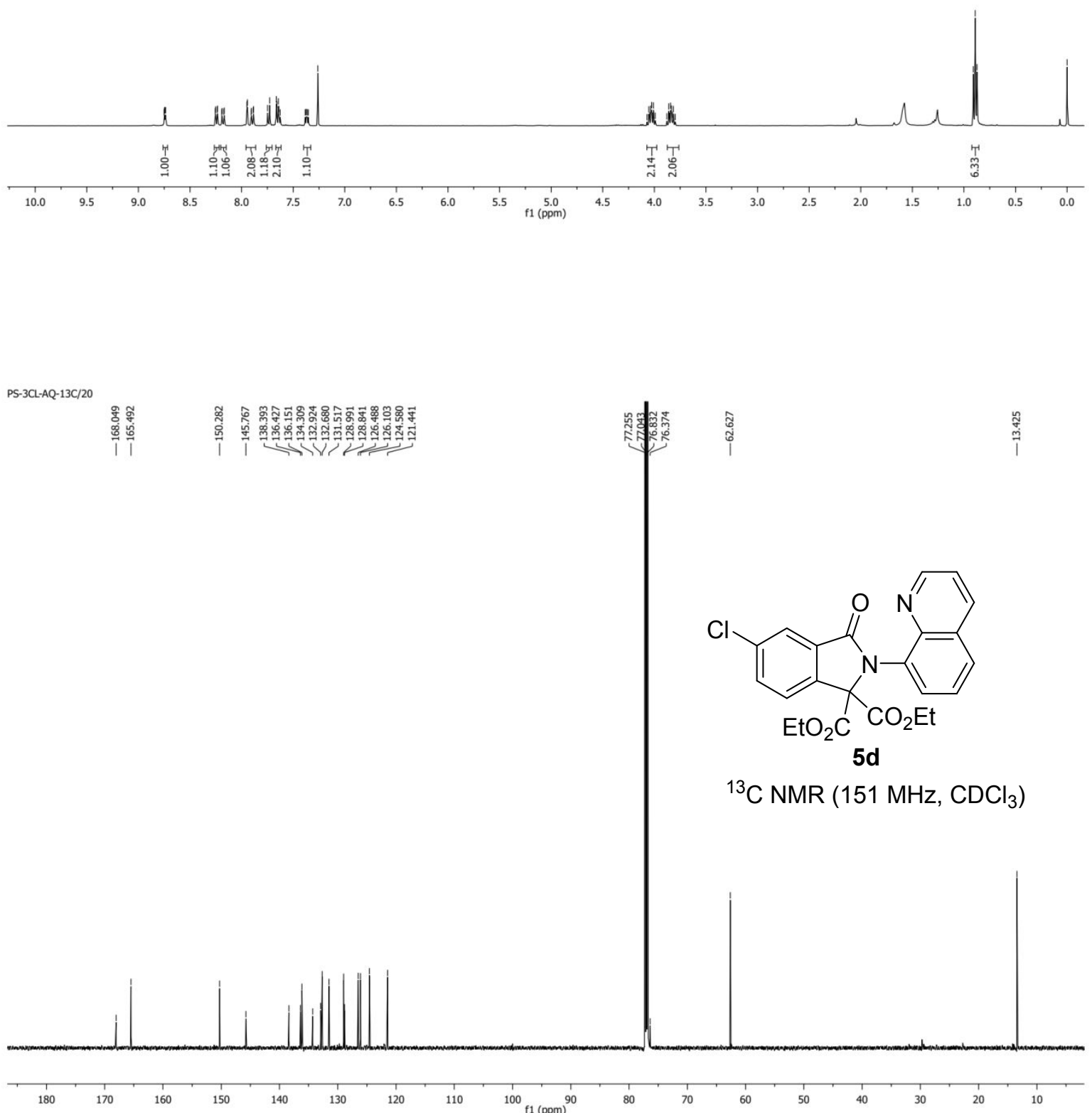


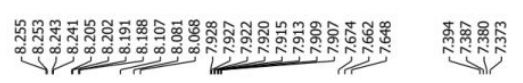
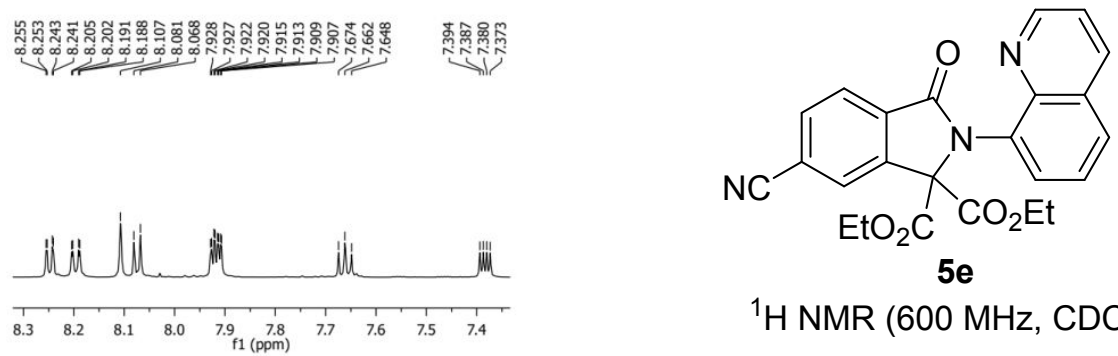

$5 e$

${ }^{1} \mathrm{H} \mathrm{NMR}\left(600 \mathrm{MHz}, \mathrm{CDCl}_{3}\right)$
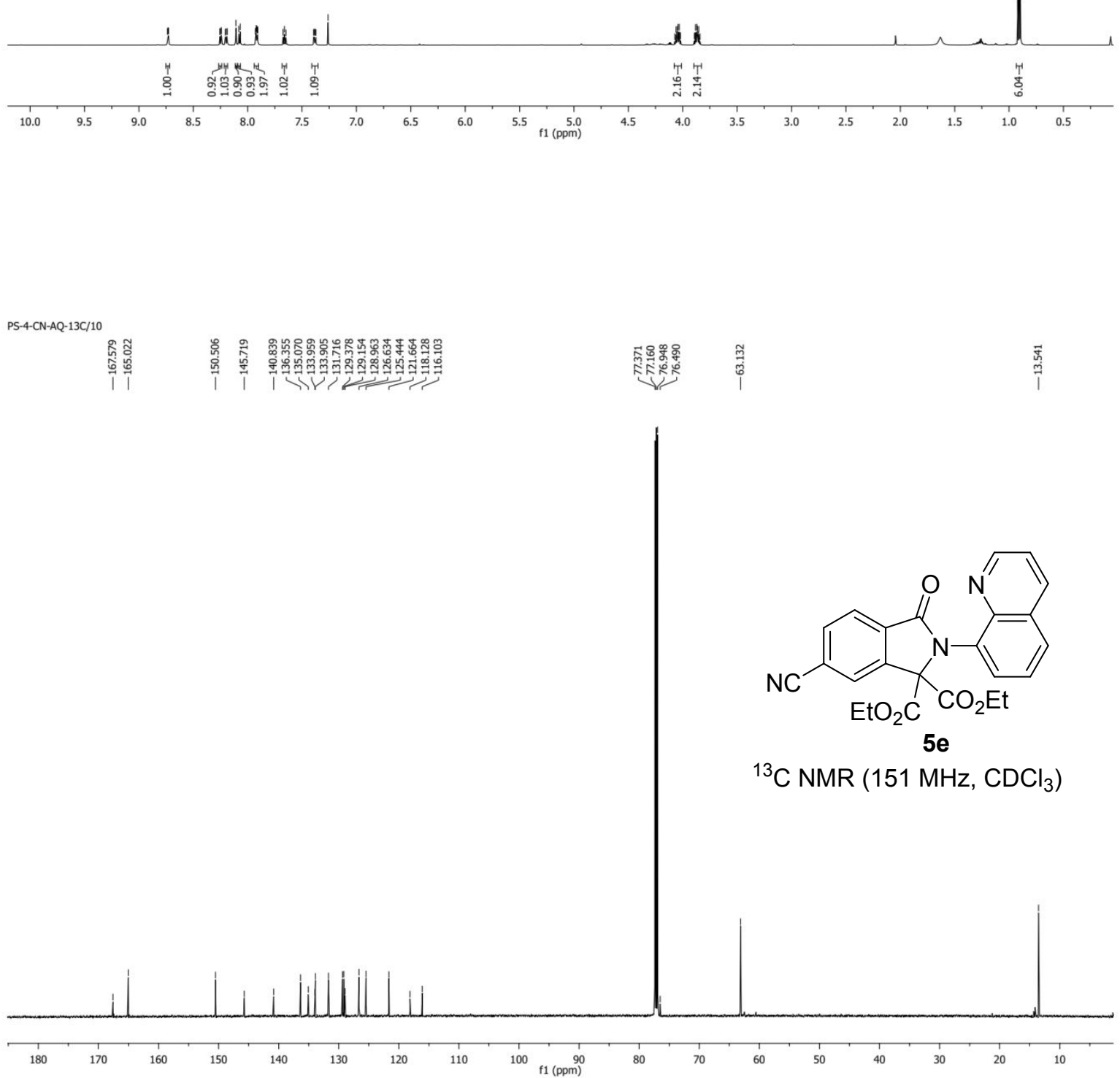


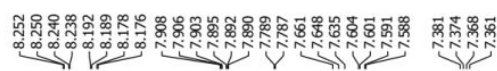

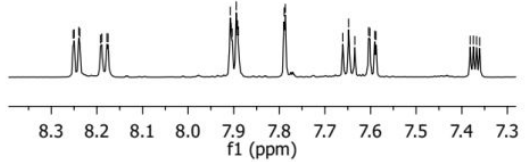

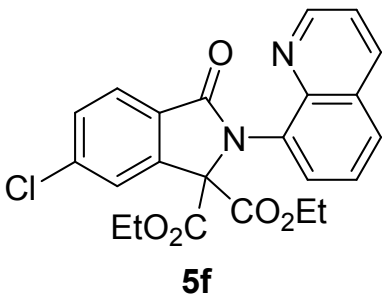

${ }^{1} \mathrm{H}$ NMR $\left(600 \mathrm{MHz}, \mathrm{CDCl}_{3}\right)$
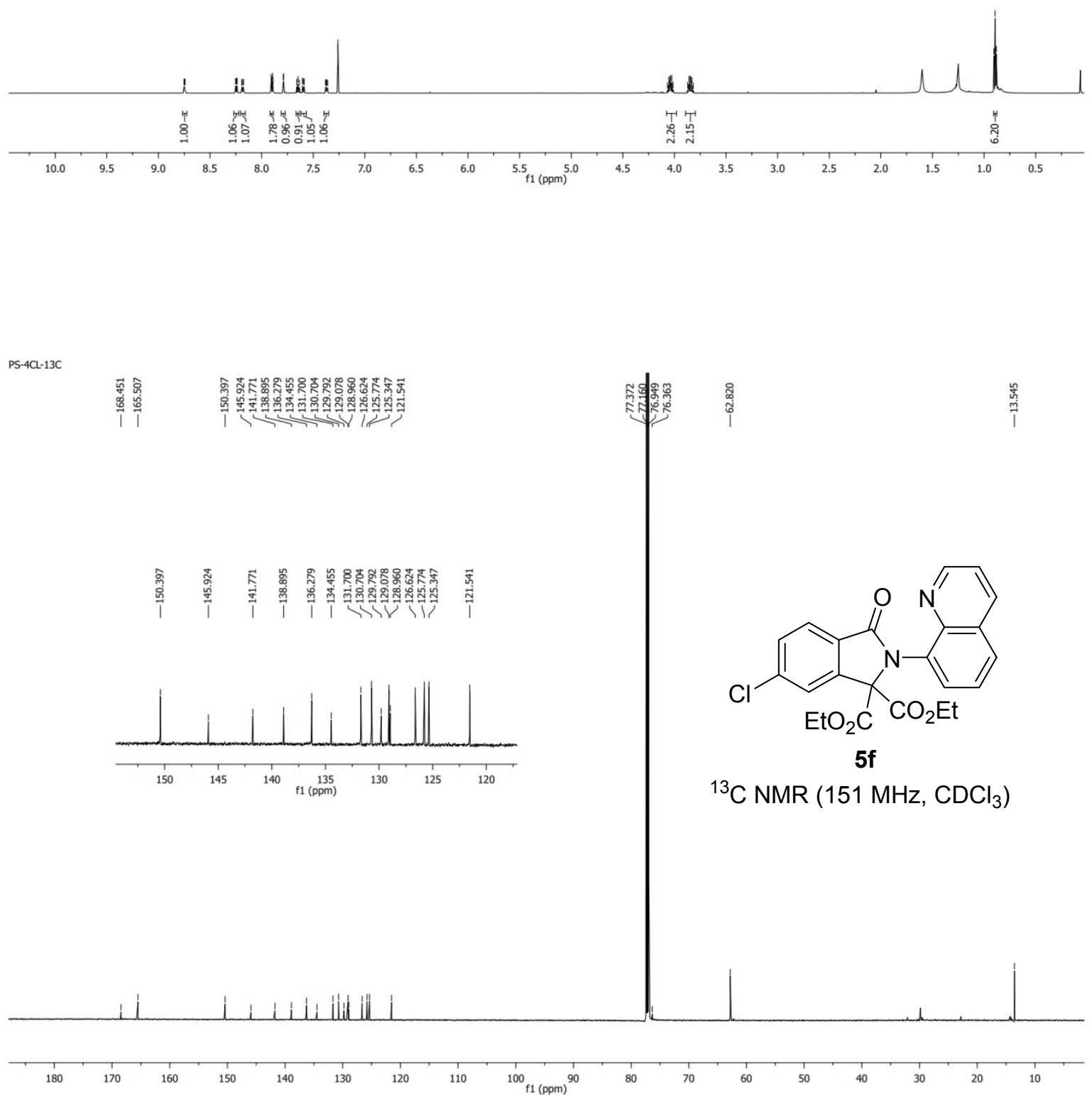


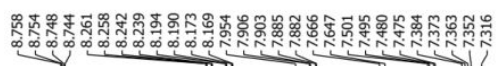

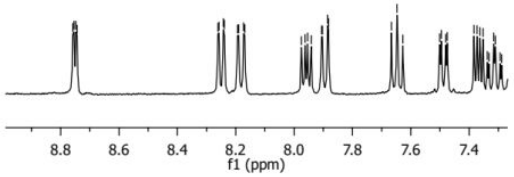<smiles>CCOC(=O)C1(C(=O)CC)c2cc(F)ccc2C(=O)N1c1cccc2cccnc12</smiles>

$5 \mathrm{~g}$
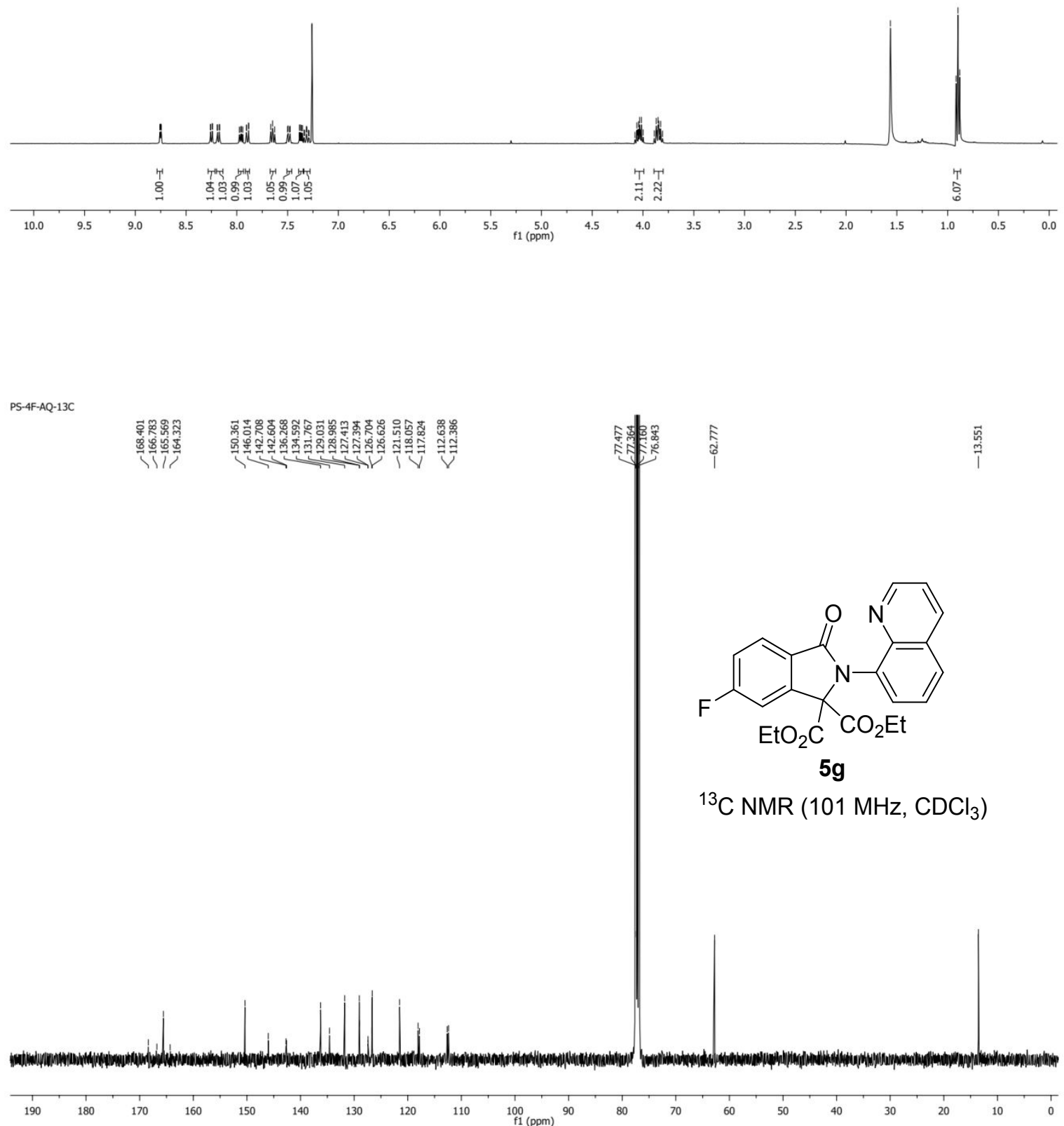

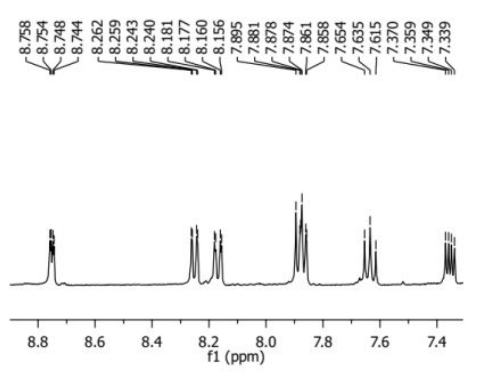<smiles>CCOC(=O)C1(C(=O)OCC)c2cc(OC)ccc2C(=O)N1c1cccc2cccnc12</smiles>

$5 \mathrm{~h}$

${ }^{1} \mathrm{H} \mathrm{NMR}\left(400 \mathrm{MHz}, \mathrm{CDCl}_{3}\right)$
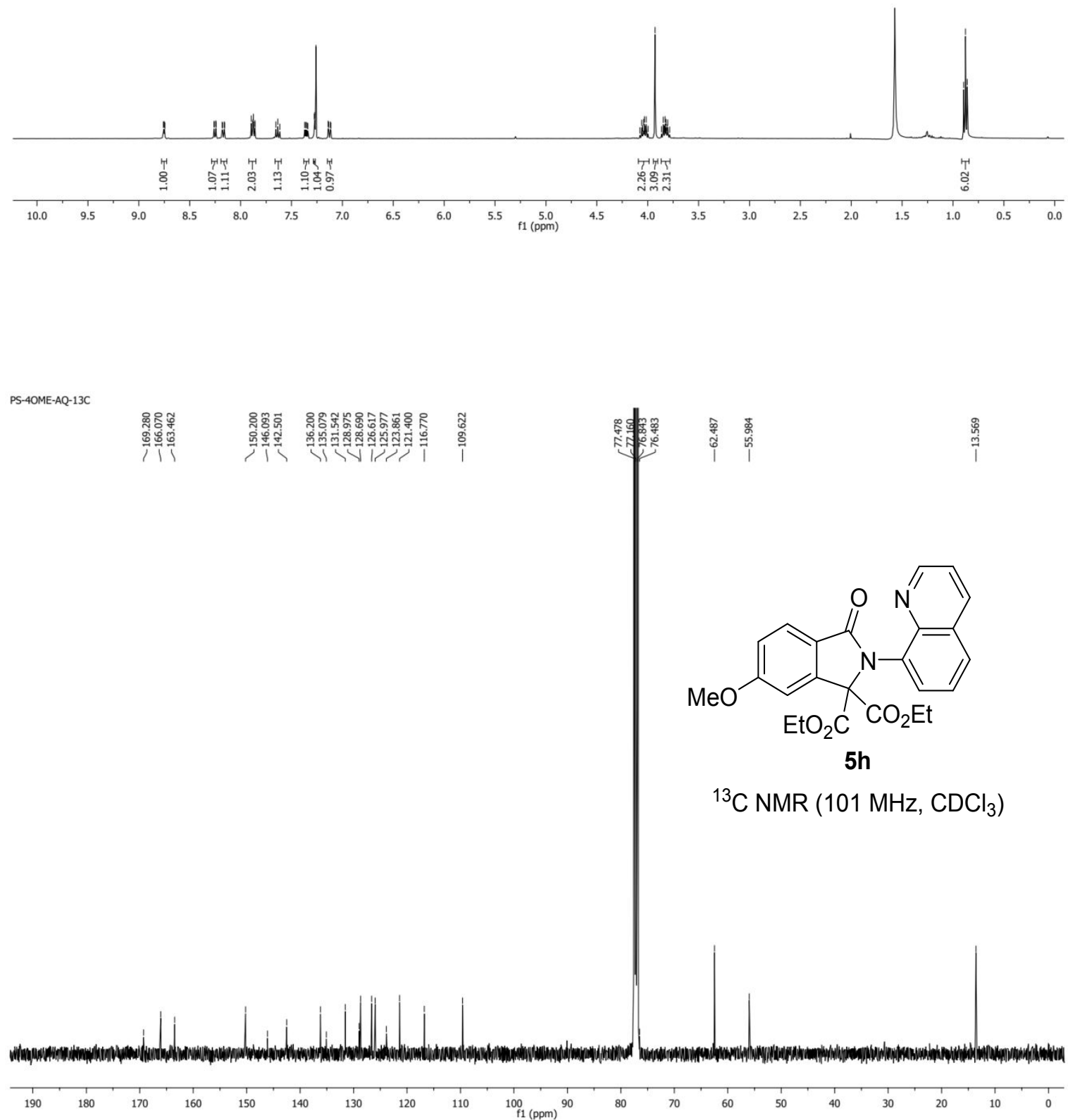

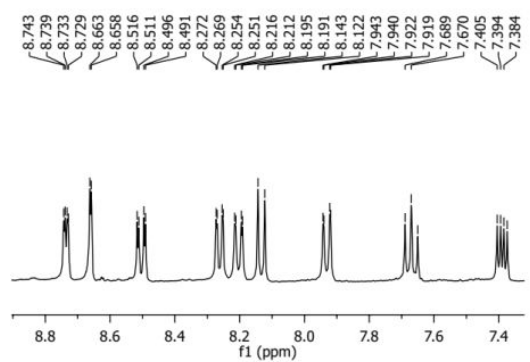<smiles>CCOC(=O)C1(C(=O)OCC)c2cc([N+](=O)[O-])ccc2C(=O)N1c1cccc2cccnc12</smiles>

$5 \mathbf{i}$

${ }^{1} \mathrm{H}$ NMR $\left(400 \mathrm{MHz}, \mathrm{CDCl}_{3}\right)$

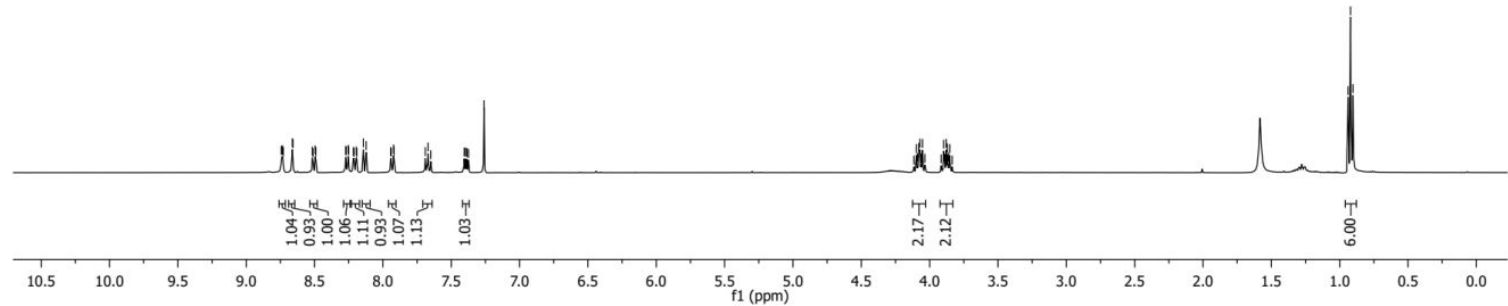

PS-4NO2-AQ-13C/10

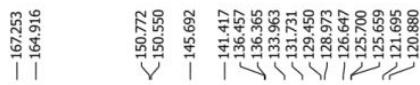

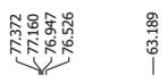

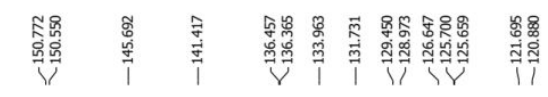

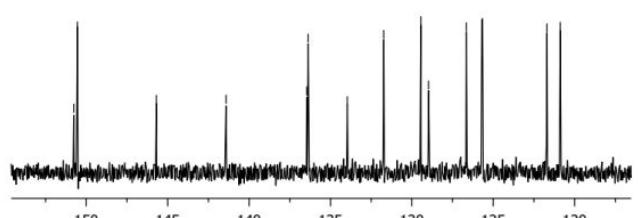

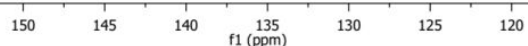

1

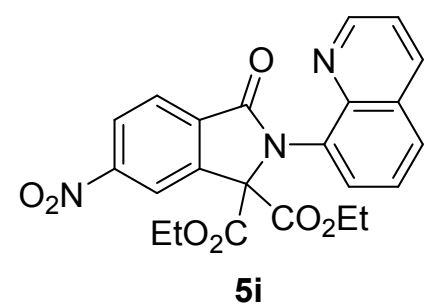

${ }^{13} \mathrm{C}$ NMR $\left(151 \mathrm{MHz}, \mathrm{CDCl}_{3}\right)$

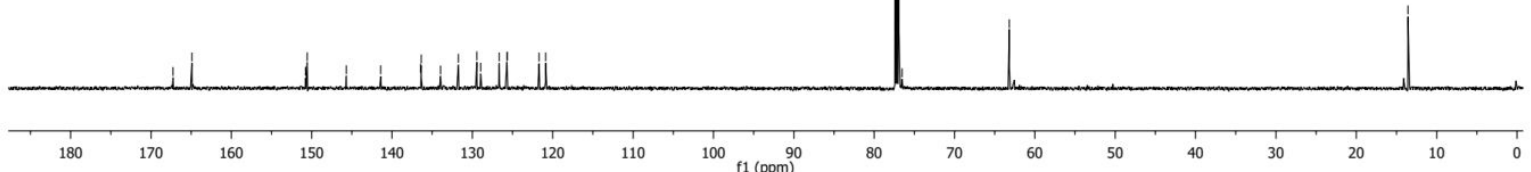




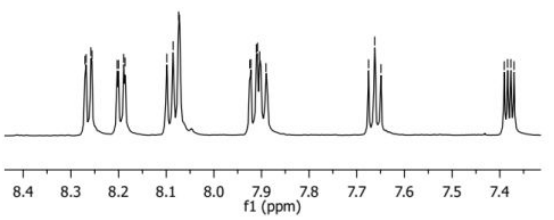<smiles>CCOC(=O)C1(C(=O)OCC)c2ccc(C(F)(F)F)cc2C(=O)N1c1cccc2cccnc12</smiles>

$5 j$

${ }^{1} \mathrm{H}$ NMR $\left(600 \mathrm{MHz}, \mathrm{CDCl}_{3}\right)$
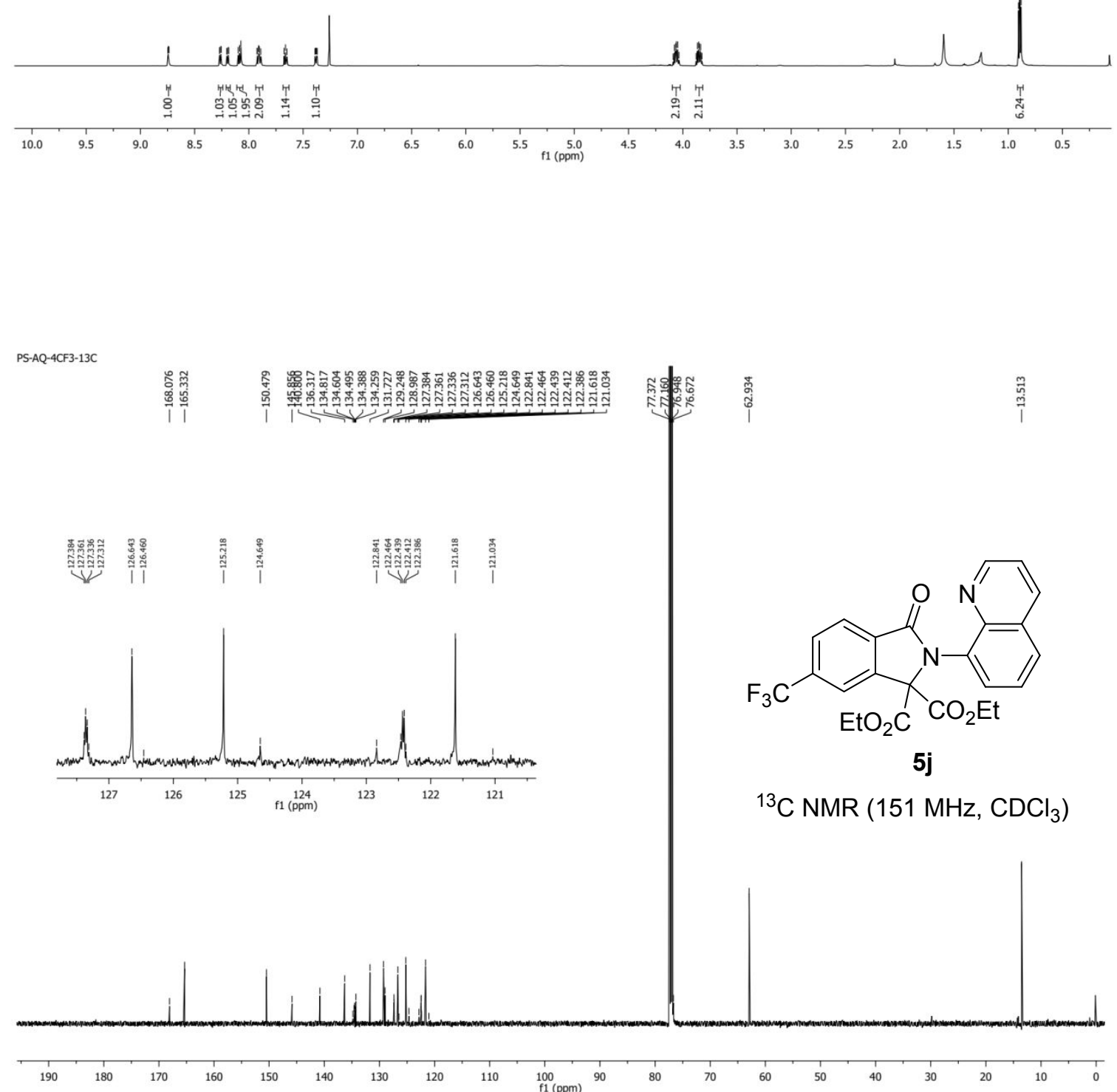


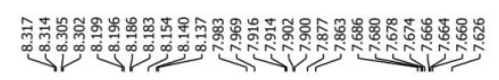

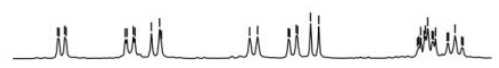

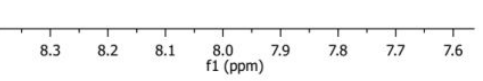<smiles>CCOC(=O)C1(C(=O)OCC)c2ccc3ccccc3c2C(=O)N1c1cccc2cccnc12</smiles>

$5 \mathbf{k}$

${ }^{1} \mathrm{H} \mathrm{NMR}\left(600 \mathrm{MHz}, \mathrm{CDCl}_{3}\right)$

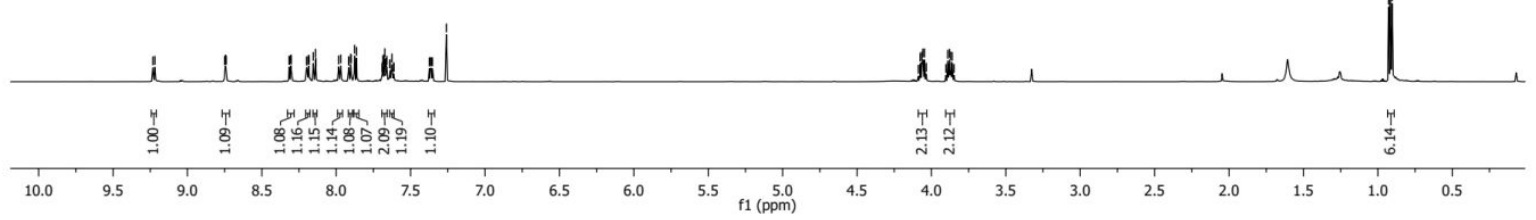

PS-1-NAP-AQ-13C/10

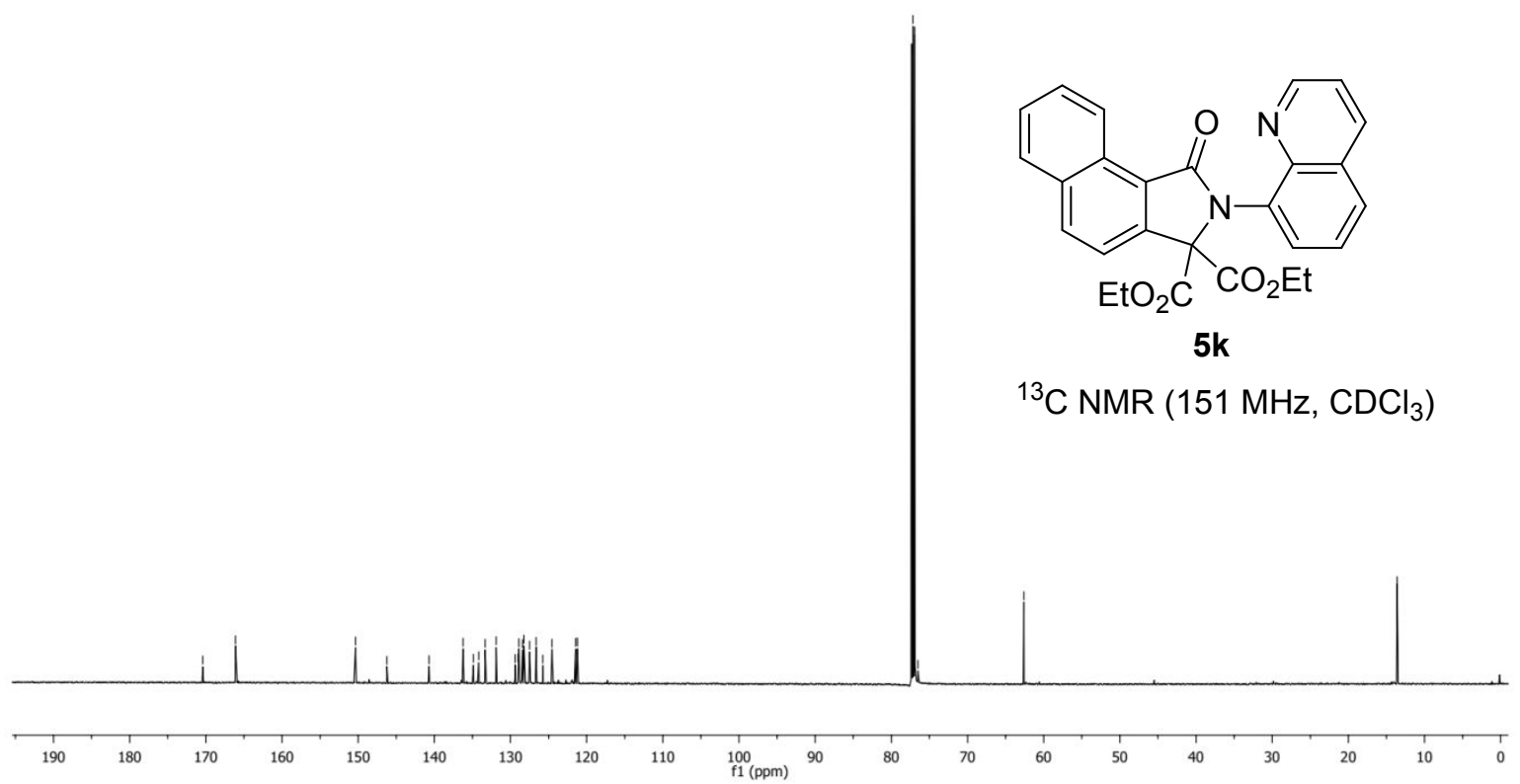




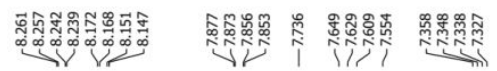

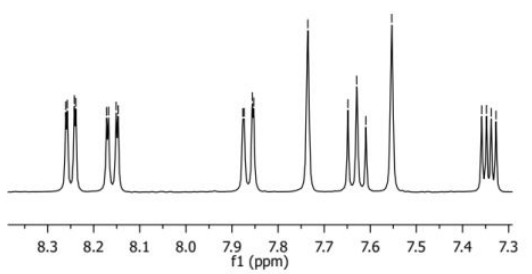<smiles></smiles>

${ }^{1} \mathrm{H}$ NMR $\left(400 \mathrm{MHz}, \mathrm{CDCl}_{3}\right)$

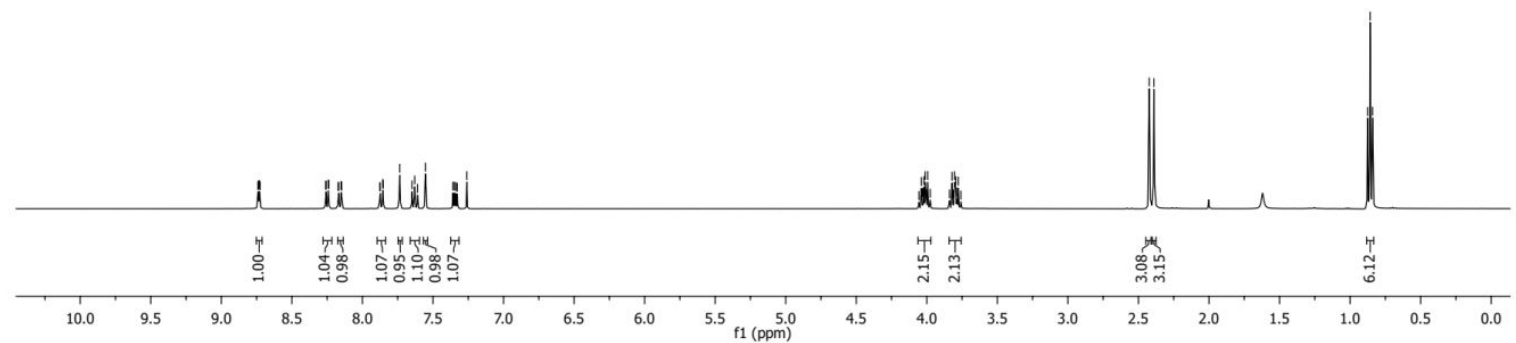

PS-3,4-ME-AQ-13C

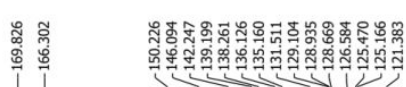

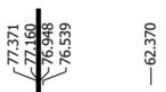

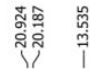<smiles>CCOC(=O)C1(C(=O)OCC)c2cc(C)c(C)cc2C(=O)N1c1cccc2cccnc12</smiles>

5

${ }^{13} \mathrm{C} \mathrm{NMR}\left(151 \mathrm{MHz}, \mathrm{CDCl}_{3}\right)$

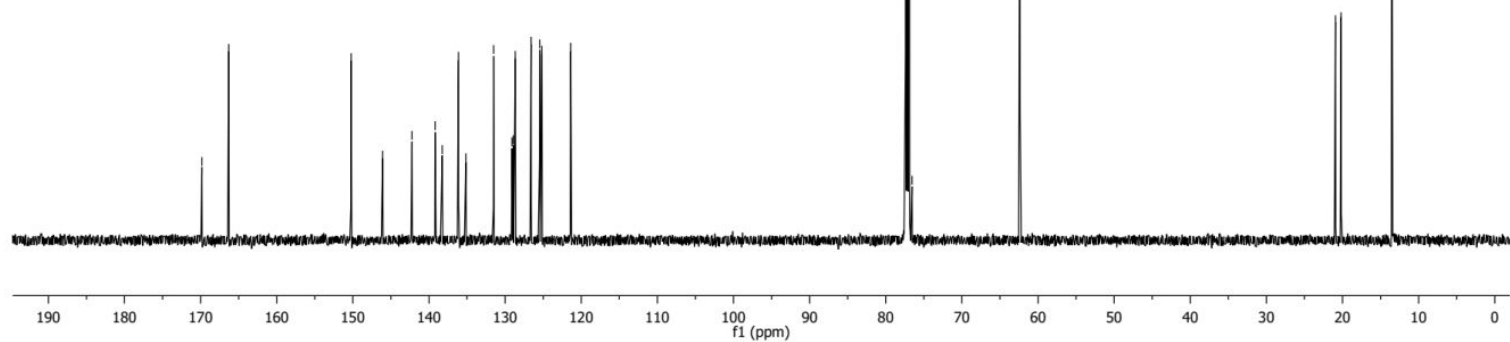




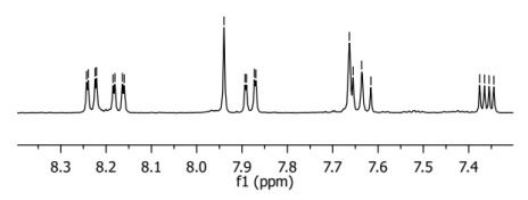<smiles>CCOC(=O)C1(C(=O)OCC)c2cc(C)c(Cl)cc2C(=O)N1c1cccc2cccnc12</smiles>

$5 \mathrm{~m}$

${ }^{1} \mathrm{H}$ NMR $\left(400 \mathrm{MHz}, \mathrm{CDCl}_{3}\right)$
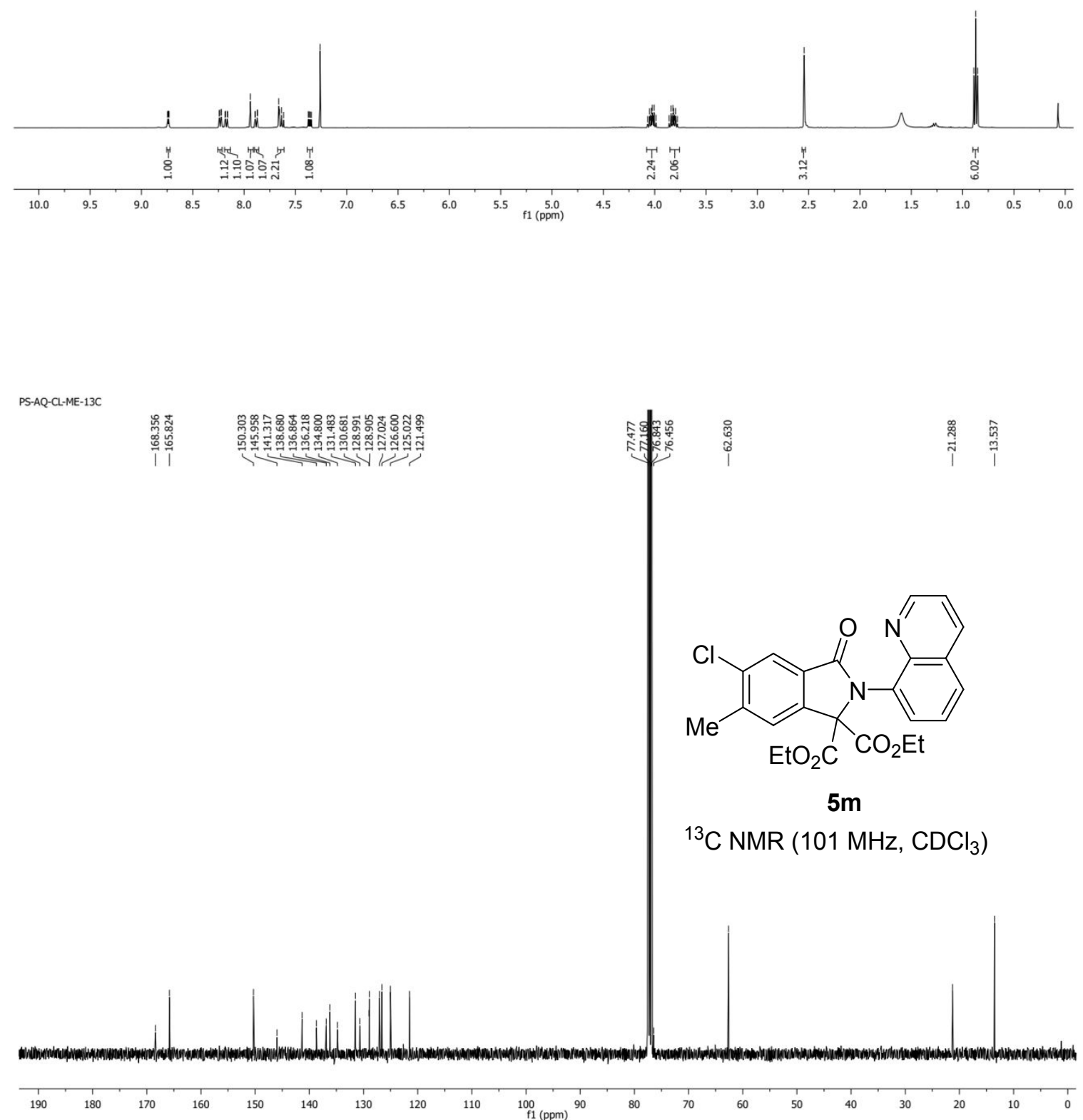


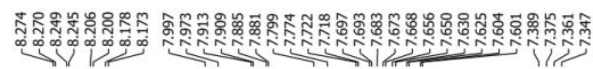

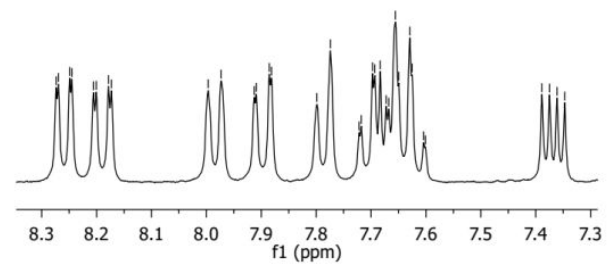

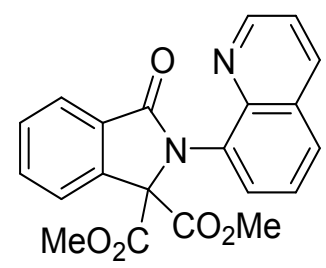

$5 q$

${ }^{1} \mathrm{H}$ NMR $\left(300 \mathrm{MHz}, \mathrm{CDCl}_{3}\right)$
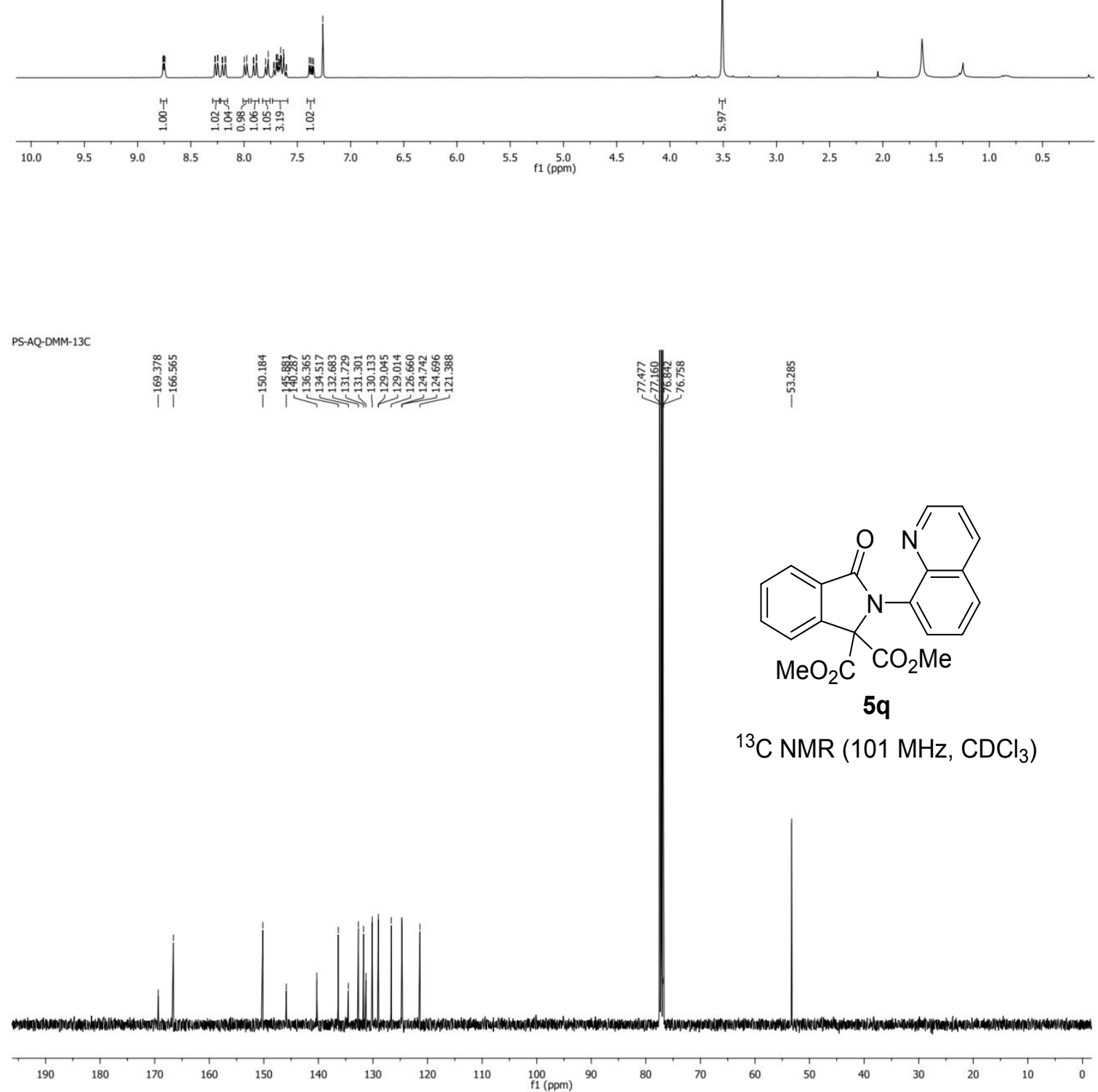


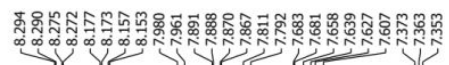

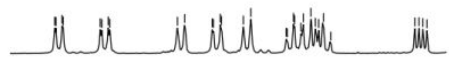<smiles>CCCOC(=O)C1(C(=O)OCC)c2ccccc2C(=O)N1c1cccc2cccnc12</smiles>

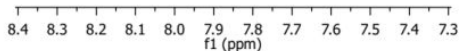

$5 r$

${ }^{1} \mathrm{H} \mathrm{NMR}\left(400 \mathrm{MHz}, \mathrm{CDCl}_{3}\right)$

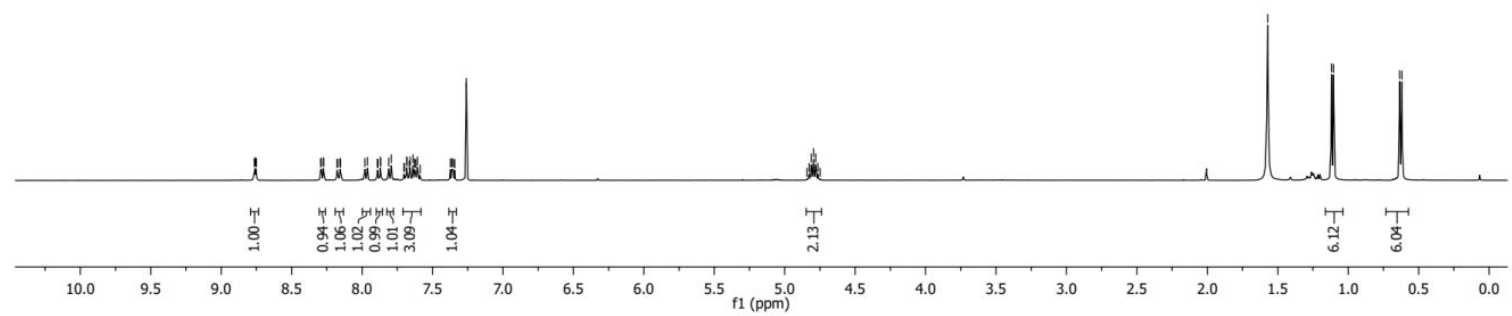

PSAQ-IPR-13C/10

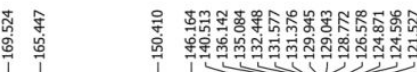

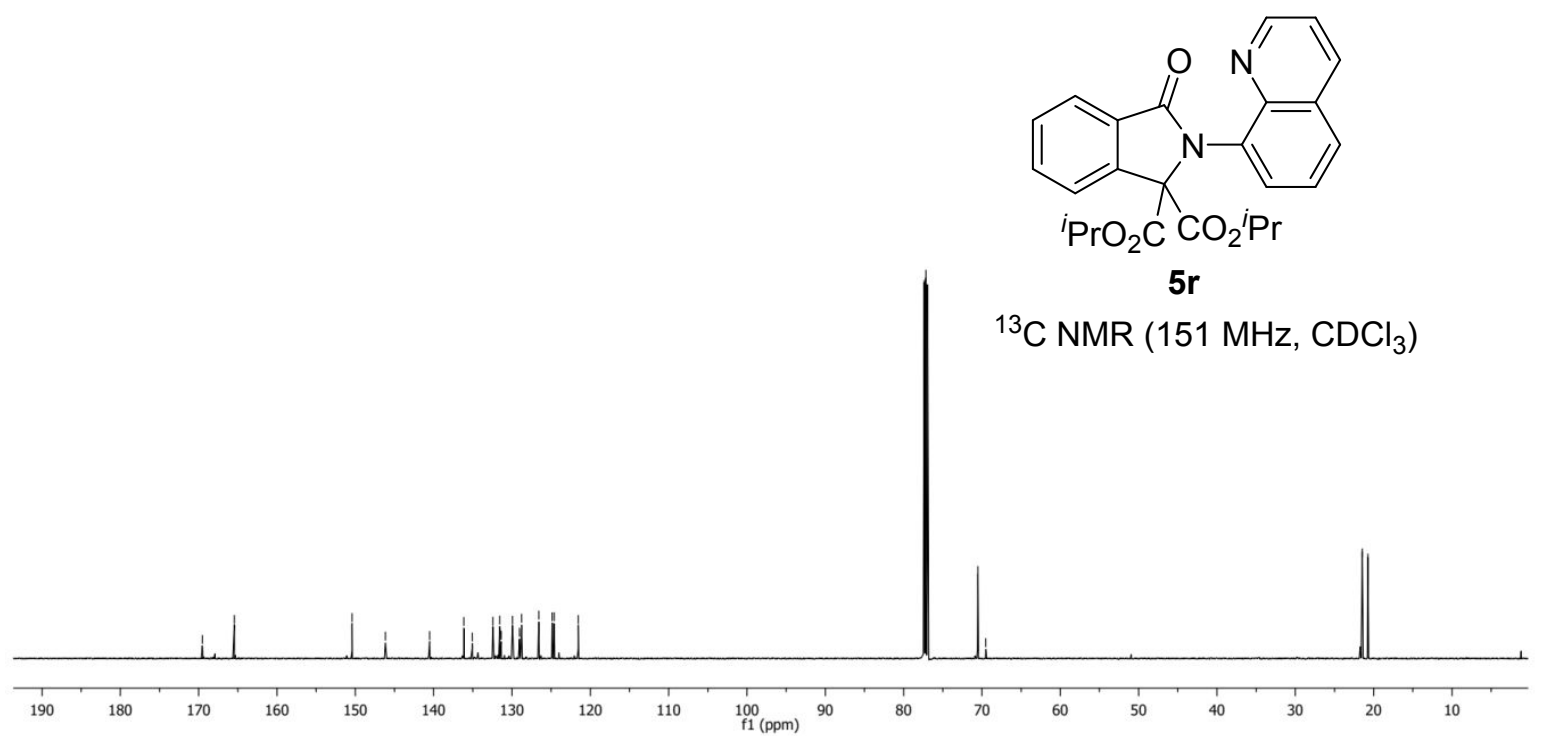


PS-AQ-TBU-MAL-1H

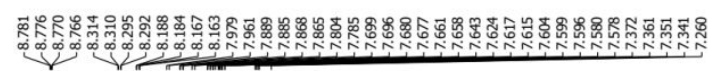

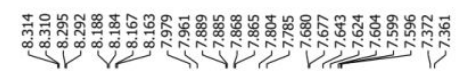

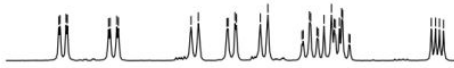

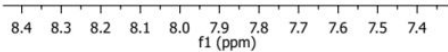

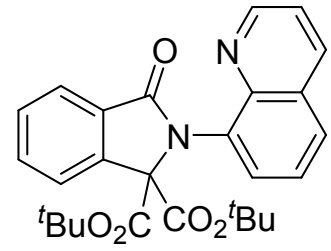

$5 s$

${ }^{1} \mathrm{H}$ NMR (400 MHz, $\mathrm{CDCl}_{3}$ )

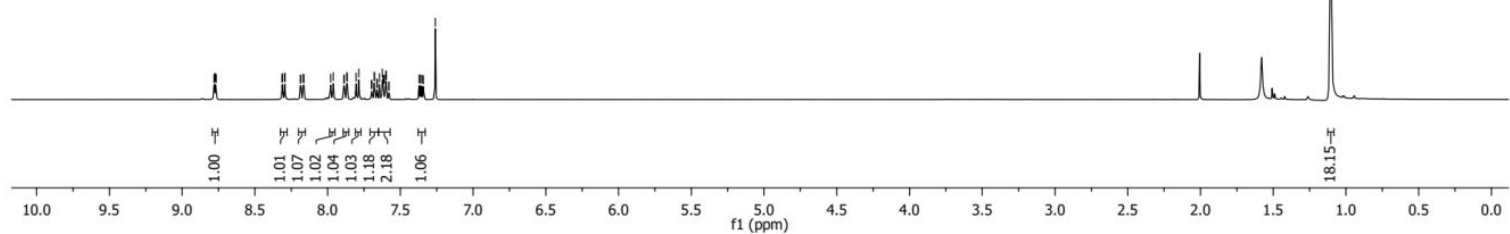

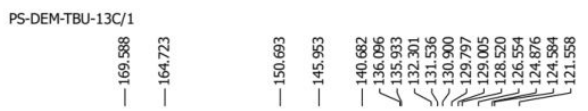
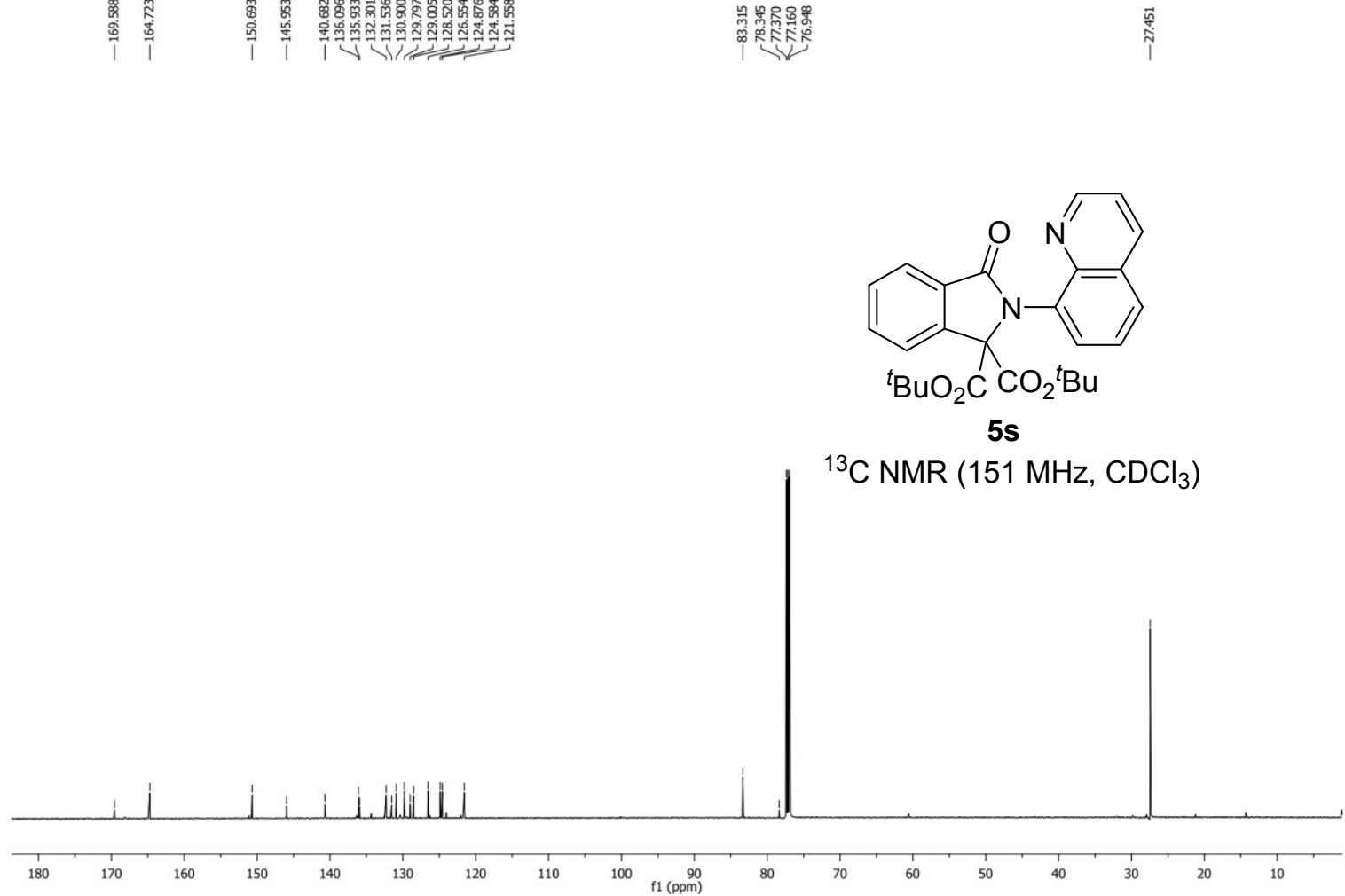


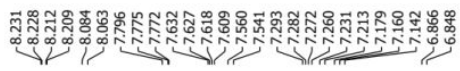

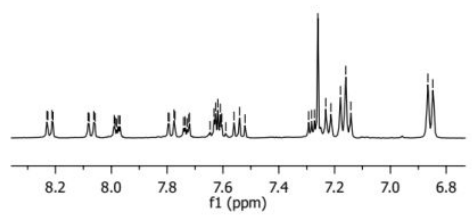

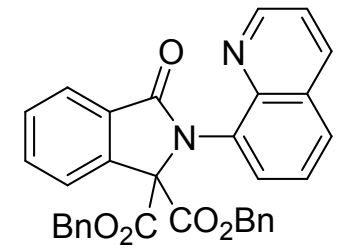

$5 \mathbf{t}$

${ }^{1} \mathrm{H}$ NMR $\left(400 \mathrm{MHz}, \mathrm{CDCl}_{3}\right)$
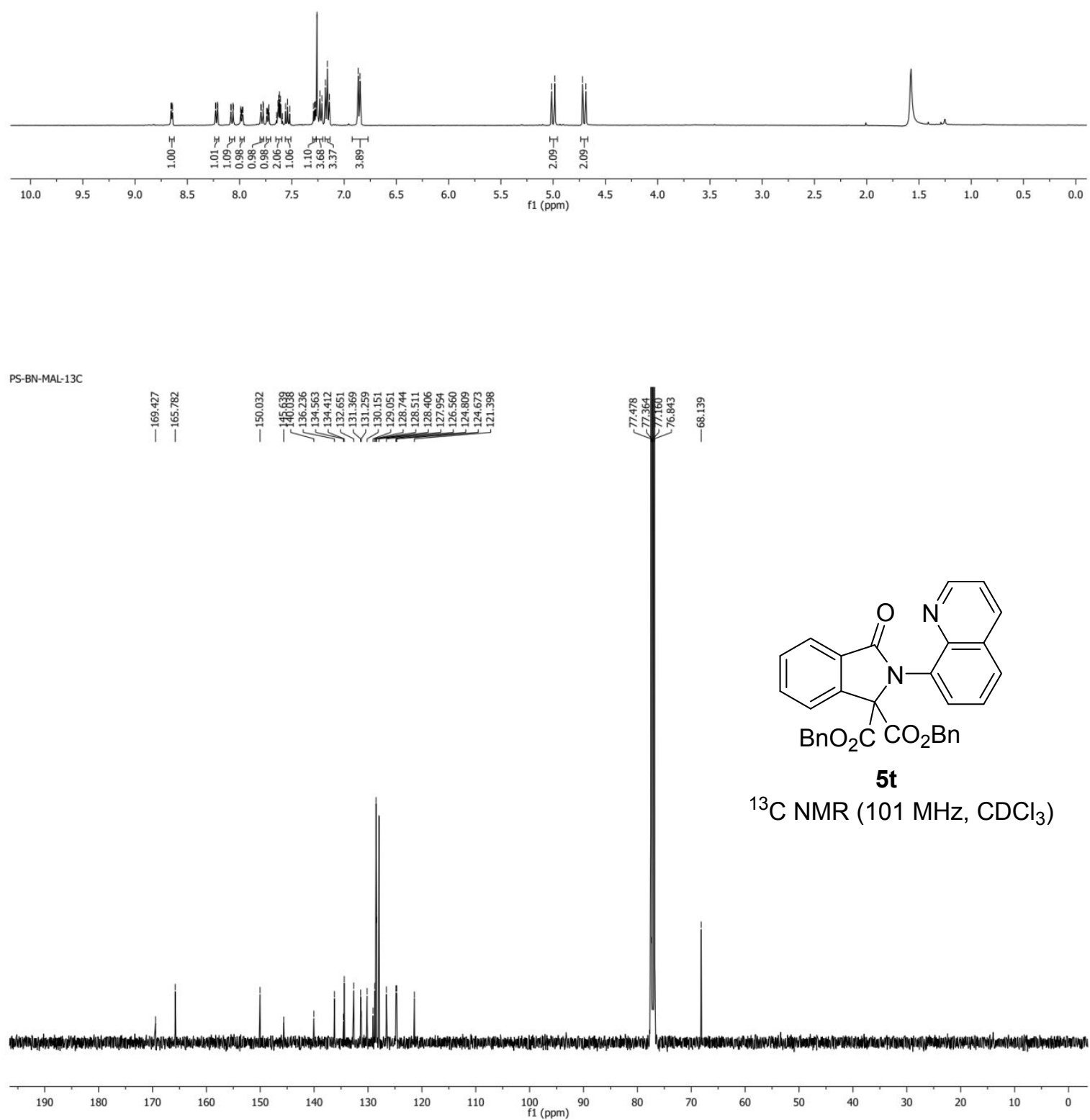


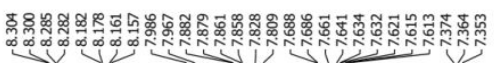

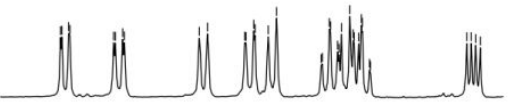

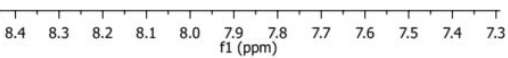

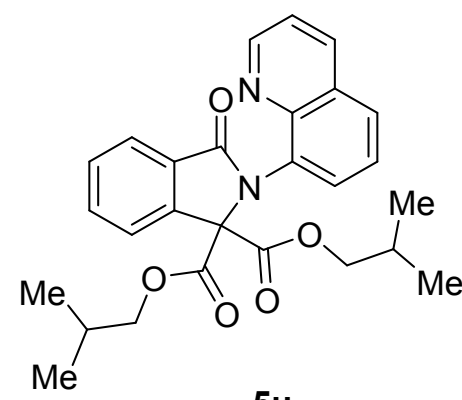

${ }^{1} \mathrm{H}$ NMR $\left(400 \mathrm{MHz}, \mathrm{CDCl}_{3}\right)$
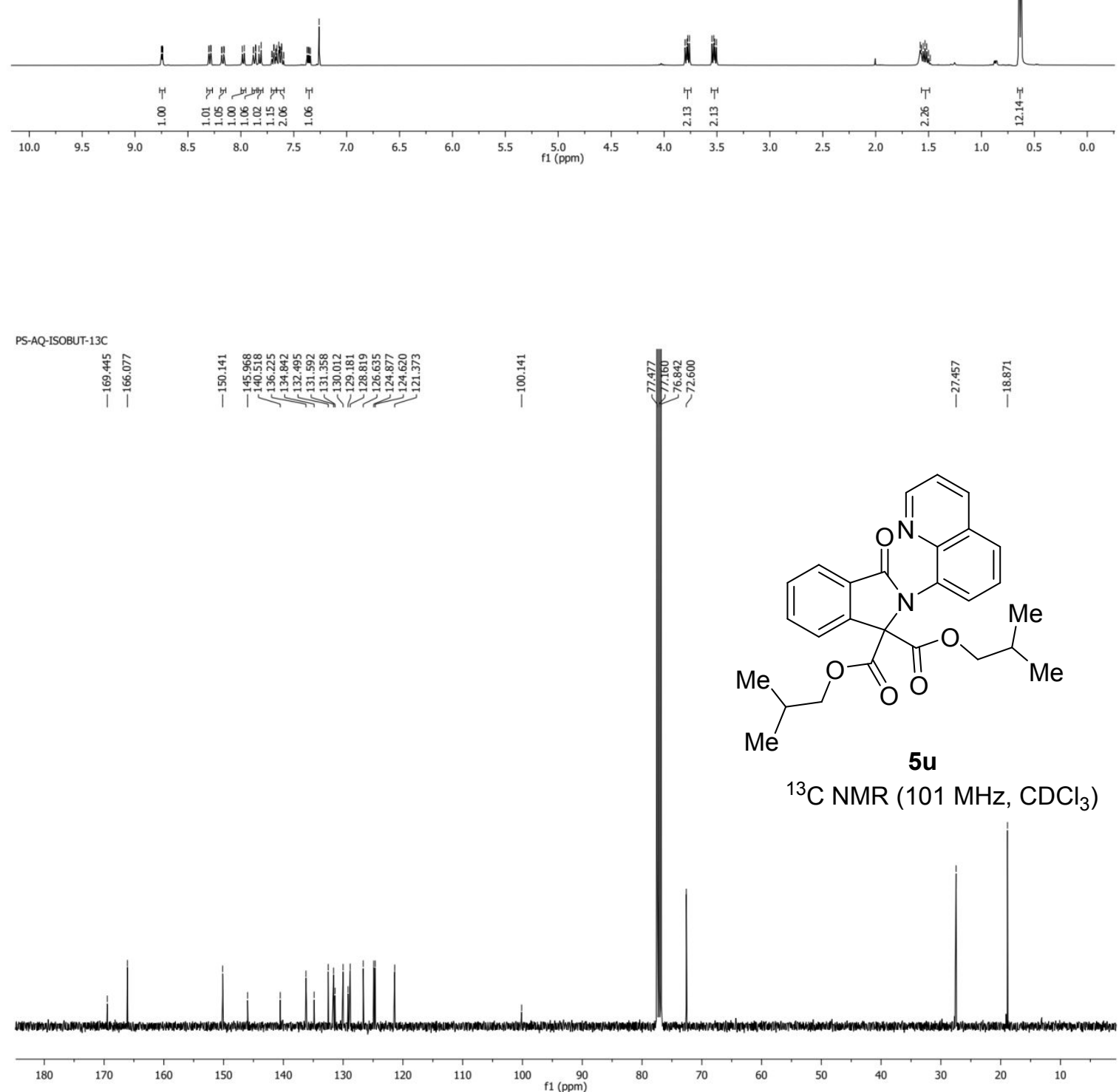


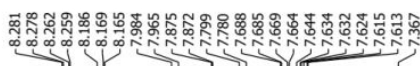

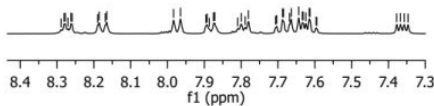

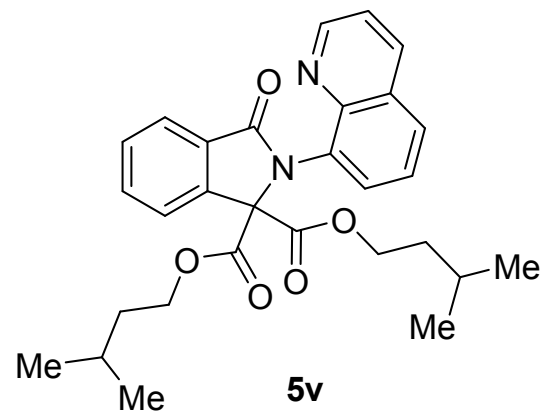

${ }^{1} \mathrm{H}$ NMR $\left(400 \mathrm{MHz}, \mathrm{CDCl}_{3}\right)$

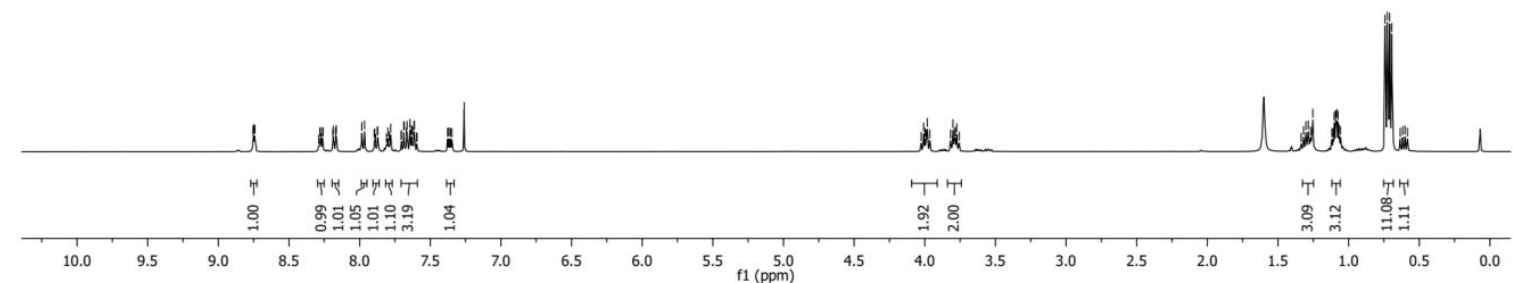

PS-AQ-ISOAM-13C
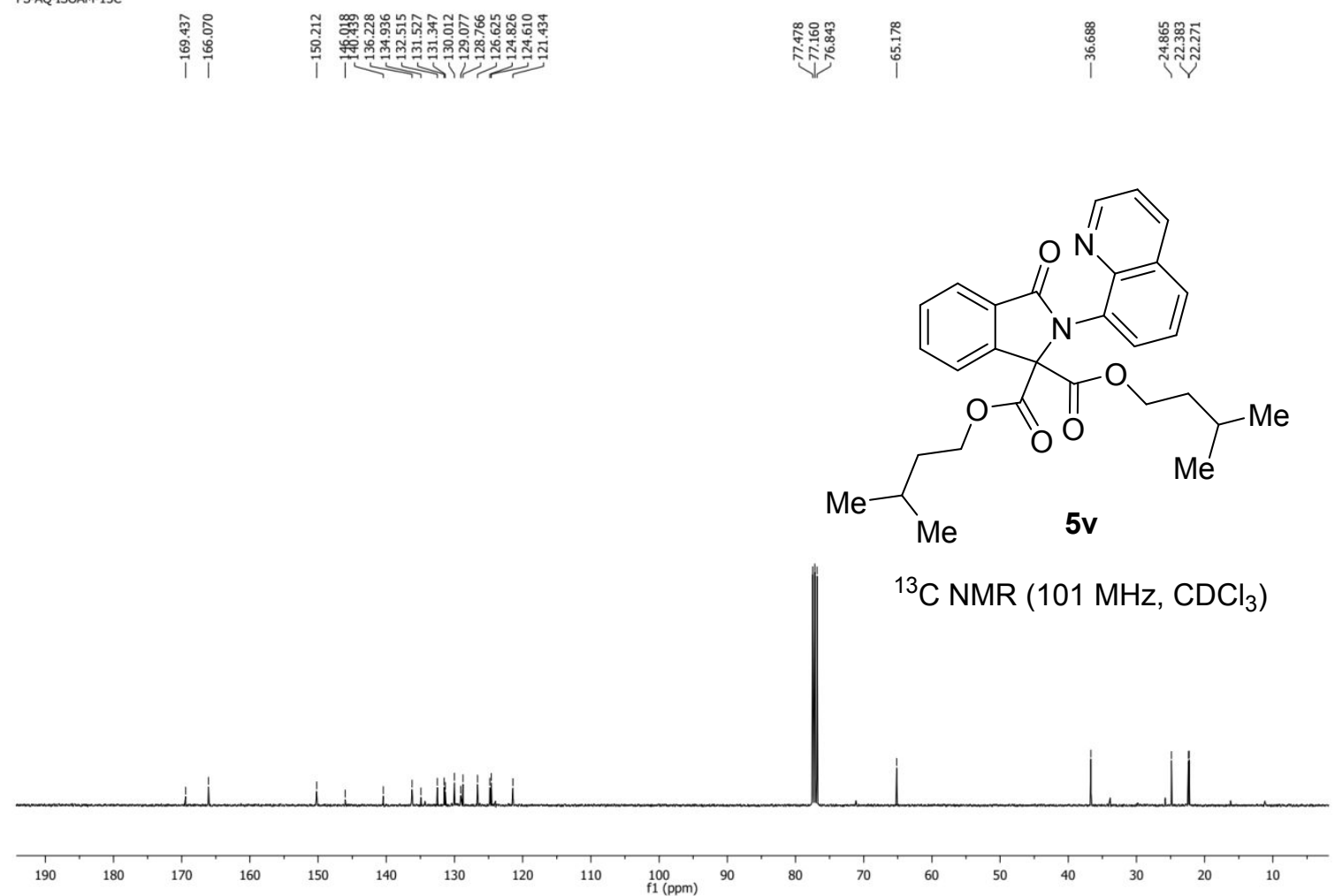


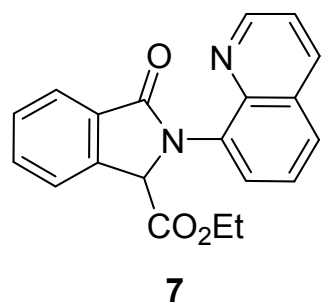

${ }^{1} \mathrm{H}$ NMR $\left(400 \mathrm{MHz}, \mathrm{CDCl}_{3}\right)$

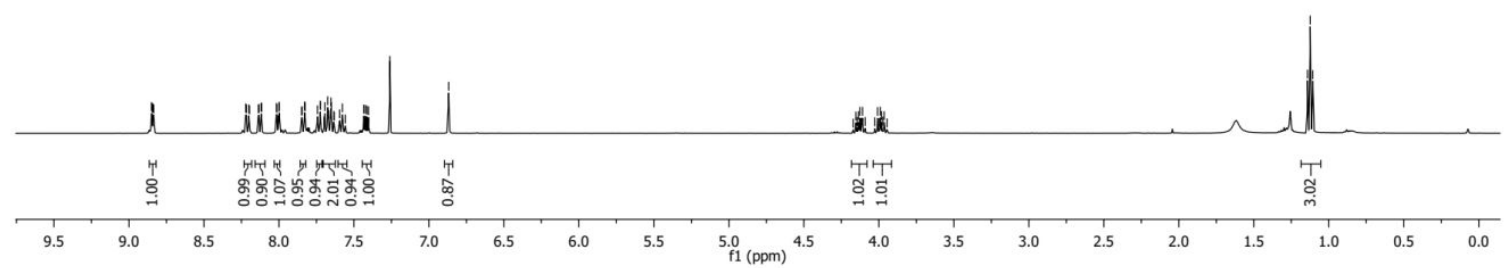

PS-AQ-KRAPCHO-13C
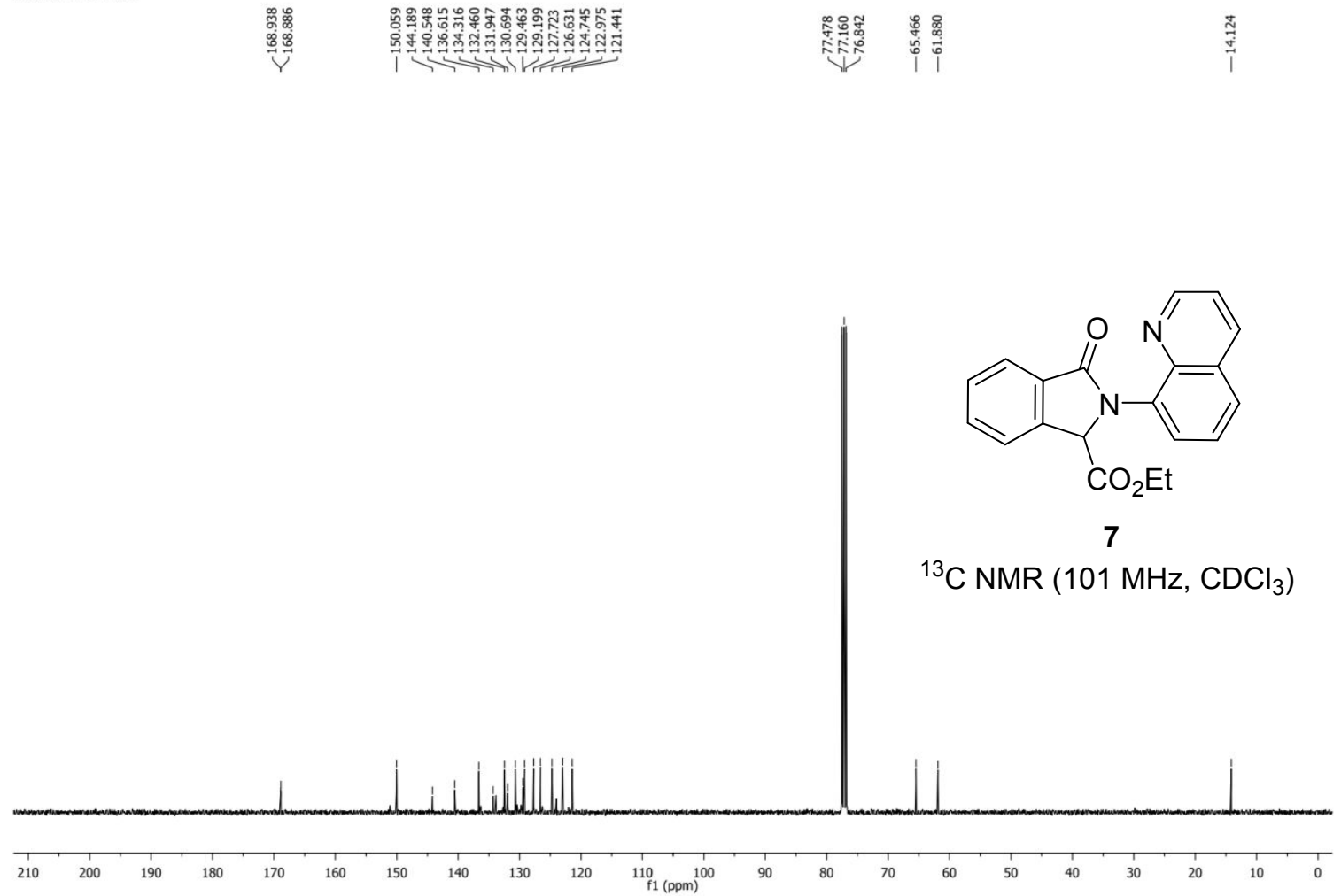


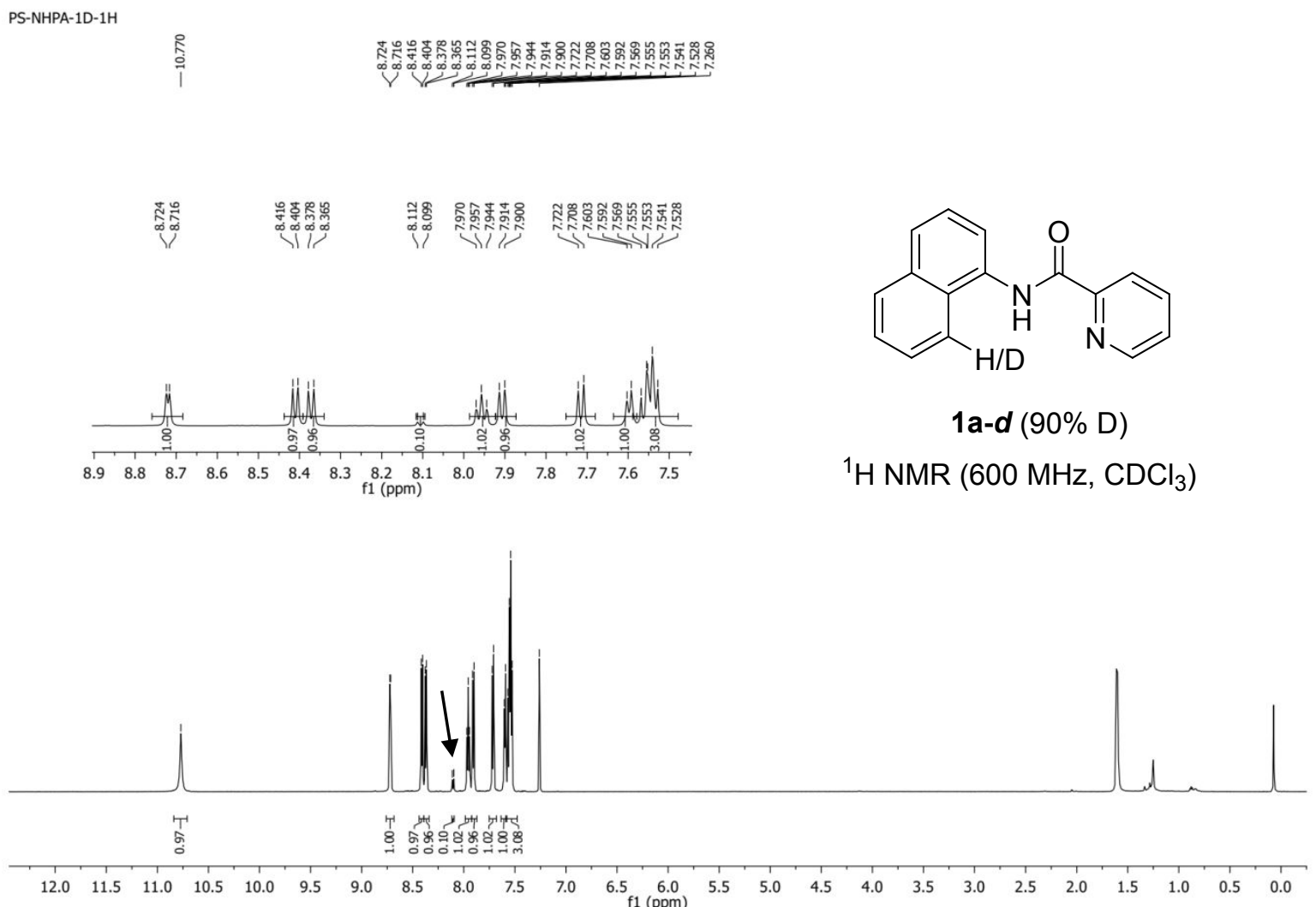

PS-NHPA-KHKD-1H-2 1

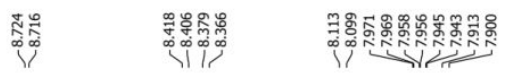

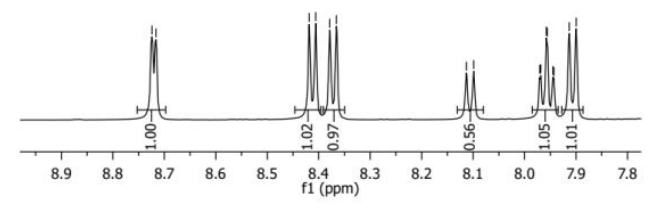<smiles>O=C(Nc1cccc2cccc(O)c12)c1ccccn1</smiles>

recovered 1a/1a-d (44\% D)

${ }^{1} \mathrm{H}$ NMR $\left(600 \mathrm{MHz}, \mathrm{CDCl}_{3}\right)$

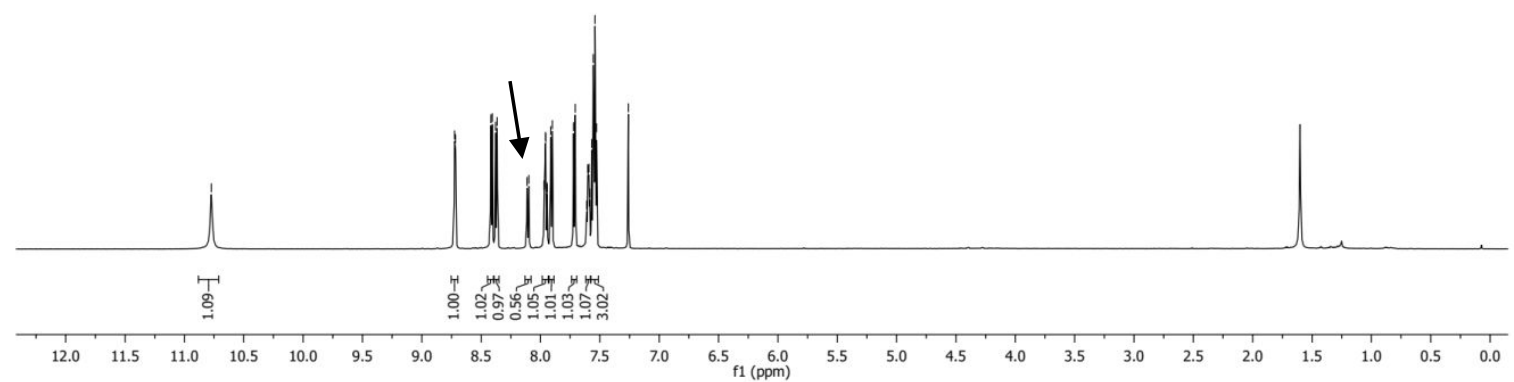

